\title{
Embedded area-constrained Willmore tori of small area in Riemannian three-manifolds I: Minimization
}

\author{
Norihisa Ikoma \\ Faculty of Mathematics and Physics \\ Institute of Science and Engineering \\ Kanazawa University \\ Kakuma, Kanazawa \\ Ishikawa 9201192, JAPAN \\ ikoma@se.kanazawa-u.ac.jp
}

\author{
Andrea Malchiodi
Scuola Normale Superiore
Piazza dei Cavalieri, 7
56126 Pisa, ITALY
andrea.malchiodi@sns.it \\ Andrea Malchiodi
Scuola Normale Superiore
Piazza dei Cavalieri, 7
56126 Pisa, ITALY
andrea.malchiodi@sns.it \\ Andrea Malchiodi
Scuola Normale Superiore
Piazza dei Cavalieri, 7
56126 Pisa, ITALY
andrea.malchiodi@sns.it \\ Andrea Malchiodi
Scuola Normale Superiore
Piazza dei Cavalieri, 7
56126 Pisa, ITALY
andrea.malchiodi@sns.it \\ Andrea Malchiodi
Scuola Normale Superiore
Piazza dei Cavalieri, 7
56126 Pisa, ITALY
andrea.malchiodi@sns.it
}

\author{
Andrea Mondino \\ Mathematics Institute \\ Zeeman Building \\ University of Warwick \\ Coventry CV4 7AL, UK \\ A.Mondino@warwick.ac.uk
}

June 15, 2018

\begin{abstract}
We construct embedded Willmore tori with small area constraint in Riemannian three-manifolds under some curvature condition used to prevent Möbius degeneration. The construction relies on a Lyapunov-Schmidt reduction; to this aim we establish new geometric expansions of exponentiated small symmetric Clifford tori and analyze the sharp asymptotic behavior of degenerating tori under the action of the Möbius group. In this first work we prove two existence results by minimizing or maximizing a suitable reduced functional, in particular we obtain embedded area-constrained Willmore tori (or, equivalently, toroidal critical points of the Hawking mass under area-constraint) in compact 3-manifolds with constant scalar curvature and in the double Schwarzschild space. In a forthcoming paper new existence theorems will be achieved via Morse theory.
\end{abstract}

Key Words: Willmore functional, Willmore tori, Hawking mass, nonlinear fourth order partial differential equations, Lyapunov-Schmidt reduction.

\section{AMS subject classification: 49Q10, 53C21, 53C42, 35J60, 83C99.}

\section{Introduction}

This is the first of a series of two papers where the construction of embedded area-constrained Willmore tori in Riemannian three-manifolds is performed under curvature conditions: here via minimization/maximization, while in the second paper 13 via Morse theory.

Let us start by introducing the Willmore functional. Given an immersion $i: \Sigma \hookrightarrow(M, g)$ of a closed (compact, without boundary) two-dimensional surface $\Sigma$ into a Riemannian 3-manifold $(M, g)$, the Willmore functional is defined by

$$
W(i):=\int_{\Sigma} H^{2} d \sigma
$$

where $d \sigma$ is the area form induced by the immersion and $H$ is the mean curvature (we adopt the convention that $H$ is the sum of the principal curvatures or, in other words, $H$ is the trace of the second fundamental form $A_{i j}$ with respect to the induced metric $\bar{g}_{i j}: H:=\bar{g}^{i j} A_{i j}$ ). 
An immersion $i$ is called Willmore surface (or Willmore immersion) if it is a critical point of the Willmore functional with respect to normal perturbations or, equivalently, if it satisfies the associated Euler-Lagrange equation

$$
\Delta_{\bar{g}} H+H|\AA|^{2}+H \operatorname{Ric}(n, n)=0,
$$

where $\Delta_{\bar{g}}$ is the Laplace-Beltrami operator corresponding to the induced metric $\bar{g},(\AA)_{i j}:=A_{i j}-\frac{1}{2} H \bar{g}_{i j}$ is the trace-free second fundamental form, $n$ is a normal unit vector to $i$, and Ric is the Ricci tensor of the ambient manifold $(M, g)$. Notice that (2) is a fourth-order nonlinear elliptic PDE in the immersion map $i$.

The Willmore functional was first introduced for immersions into the Euclidean space in 20's and 30's by Blaschke and Thomsen, who were looking for a conformal invariant theory which included minimal surfaces. Since minimal surfaces trivially satisfy (2) and - as they proved - $W$ is invariant under Möbius transformations in the Euclidean space, they detected the class of Willmore surfaces as the natural conformally invariant generalization of minimal surfaces and called them conformal minimal surfaces. The topic was then rediscovered in the 60 's by Willmore [44, who proved that the global minimum among all closed immersed surfaces was strictly achieved by round spheres and conjectured that the genus one minimizer was the Clifford torus (and its images via Möbius transformations). This long standing conjecture has been recently proved by Marques-Neves 25] by min-max techniques (partial results towards the Willmore conjecture were previously obtained by Li-Yau [23], Montiel-Ros [34], Ros [38, Topping [40] and others, and that a crucial role in the proof of the conjecture is played by a result of Urbano [41]). Let us also mention the fundamental works on the Willmore functional by Simon [39] who proved the existence of a smooth genus one minimizer of $W$ in $\mathbb{R}^{m}$ (later generalized to higher genus by Bauer-Kuwert [5] who were inspired by a paper of Kusner [14; an alternative proof of these results has been recently given by Rivière [37] using the so called parametric approach), by Kuwert-Schätzle [16] and by Rivière 36.

Probably because of the richness of the symmetries preserving the class of Willmore surfaces and because of the simplicity and the universality of its definition, the Willmore functional shows up in very different fields of sciences and technology. It appears for instance in biology in the study of lipid bilayer cell membranes under the name Helfrich energy, in general relativity as being the main term in the so called Hawking Mass, in string theory in high energy physics concerning the definition of Polyakov's extrinsic action, in elasticity theory this as a free energy of the non-linear plate Birkhoff theory, in optics, lens design, etc.

Let us stress that all the aforementioned results about Willmore surfaces concern immersions into Euclidean space (or, equivalently by conformal invariance, for immersions into a round sphere); the literature about Willmore immersions into curved Riemannian manifolds, which has interest in applications as it might model non-homogeneous environments, is much more recent: the first existence result was established in 28] where the third author constructed embedded Willmore spheres in a perturbative setting (see also [29], and the more recent work [10 in collaboration with Carlotto; related perturbative results under area constraint have been obtained by Lamm-Metzger-Schulze [19, by Lamm-Metzger [17]-[18, and by the third author in collaboration with Laurain [21]).

The global problem, i.e. the existence of smooth immersed spheres minimizing quadratic curvature functionals in compact Riemannian three-manifolds, was then studied by the third author in collaboration with Kuwert and Schygulla in [15] (see also [33] for the non compact case). In collaboration with Rivière [31]-32], the third author developed the necessary tools for the calculus of variations of the Willmore functional in Riemannian manifolds and proved existence of area-constrained Willmore spheres in homotopy classes (as well as the existence of Willmore spheres under various assumptions and constraints).

Since all the above existence results in Riemannian manifolds concern spherical surfaces, a natural question is about the existence of higher genus Willmore surfaces in general curved spaces; in particular we will focus here on the genus one case.

Let us mention that if the ambient space has some special symmetry then the Willmore equation (22) simplifies and one can construct explicit examples (see for instance the paper of Wang [42] in case 
the ambient manifold is a product and the work of Barros-Ferrández-Lucas-Merono 4 for the case of warped product metrics); we also mention the work of Chen-Li [11 where the existence of stratified weak branched immersions of arbitrary genus minimizing quadratic curvature functionals under various constraints is investigated.

The goal of the present (and the subsequent [13]) work is to construct smooth embedded Willmore tori with small area constraint in Riemannian three-manifolds, under some curvature condition but without any symmetry assumption. Let us recall that area-constrained Willmore surfaces satisfy the equation

$$
\Delta_{\bar{g}} H+H|\AA|^{2}+H \operatorname{Ric}(n, n)=\lambda H,
$$

for some $\lambda \in \mathbb{R}$ playing the role of Lagrange multiplier. These immersions are naturally linked to the Hawking mass

$$
m_{H}(i):=\frac{\sqrt{\operatorname{Area}(i)}}{64 \pi^{3 / 2}}(16 \pi-W(i)),
$$

since, clearly, the critical points of the Hawking mass under area constraint are exactly the areaconstrained Willmore immersions (see [7, 12, 19] and the references therein for more material about the Hawking mass).

The main result of this paper is the following (recall that the area of the Clifford torus in the Euclidean space is $\left.4 \sqrt{2} \pi^{2}\right)$.

Theorem 1.1. Let $(M, g)$ be a compact 3-dimensional Riemannian manifold. Denote by Ric and Sc the Ricci and the scalar curvature of $(M, g)$ respectively, and suppose either

$$
3 \sup _{P \in M}\left(\operatorname{Sc}_{P}-\inf _{|\nu|_{g}=1} \operatorname{Ric}_{P}(\nu, \nu)\right)>2 \sup _{P \in M} \operatorname{Sc}_{P},
$$

or else

$$
3 \inf _{P \in M}\left(\operatorname{Sc}_{P}-\sup _{|\nu|_{g}=1} \operatorname{Ric}_{P}(\nu, \nu)\right)<2 \inf _{P \in M} \operatorname{Sc}_{P} .
$$

Then there exists $\varepsilon_{0}>0$ such that for every $\varepsilon \in\left(0, \varepsilon_{0}\right)$ there exists a smooth embedded Willmore torus in $(M, g)$ with constrained area equal to $4 \sqrt{2} \pi^{2} \varepsilon^{2}$.

More precisely, these surfaces are obtained as normal graphs over exponentiated (Möbius transformations of) Clifford tori and the corresponding graph functions, once dilated by a factor $1 / \varepsilon$, converge to 0 in $C^{4, \alpha}$-norm as $\varepsilon \rightarrow 0$ with decay rate $O\left(\varepsilon^{2}\right)$.

Remark 1.2. If both the conditions (3) and (4) are satisfied then there exist at least two smooth embedded Willmore tori in $(M, g)$ with constrained area equal to $4 \sqrt{2} \pi^{2} \varepsilon^{2}$ (see Remark 5.1 for the proof).

The assumptions of Theorem 1.1 are rather mild and allow to include a large class of examples, as explained in the next corollaries and remarks. See also Remark 4.4 for an interpretation of the quantities in (3) and (4) in terms of sectional curvatures.

Corollary 1.3. Let $(M, g)$ be a compact 3-dimensional manifold with constant scalar curvature. Then there exists $\varepsilon_{0}>0$ such that for every $\varepsilon \in\left(0, \varepsilon_{0}\right)$ there exist at least two smooth embedded Willmore tori in $(M, g)$ with constrained area equal to $4 \sqrt{2} \pi^{2} \varepsilon^{2}$.

Corollary 1.3 easily follows by Remark 1.2 once we observe that if $\mathrm{Sc} \equiv S \in \mathbb{R}$ and if $(M, g)$ does not have constant sectional curvature, then Schur's lemma implies the existence of $P \in M$ at which $(M, g)$ is not isotropic. With the aid of the expressions in Remark 4.4, both the assumptions (3) and (4) are satisfied. On the other hand, if $(M, g)$ has constant sectional curvature $\bar{K} \in \mathbb{R}$ then it is conformally equivalent to (a quotient) of the Euclidean space (indeed either it is a quotient of the three-sphere $\mathbb{S}^{3}$ or 
of the Hyperbolic three-space $\mathbb{H}^{3}$ both conformally equivalent to the Euclidean three-space). Now it is well known that the functional

$$
W_{c n f}(i):=\int_{\Sigma}\left[H^{2}+4 \bar{K}\right] d \sigma=W(i)+4 \bar{K} \operatorname{Area}(i)
$$

is conformally invariant (see for instance [43]). Of course the area-constrained critical points of $W$ are exactly the area-constrained critical points of $W_{c n f}$, but then by conformal invariance it follows that Euclidean Clifford tori correspond to area-constrained Willmore tori in the space form.

Remark 1.4. The class of compact 3-manifolds with constant scalar curvature include many remarkable examples of ambient spaces which play an important role in contemporary surface theory. Trivial cases are compact quotients of space forms (notice that the same existence result applies to the standard non compact space forms as explained below), but more generally any homogeneous three manifold has of course constant scalar curvature. Examples of compact homogeneous spaces are $\mathbb{S}^{2} \times \mathbb{S}^{1}$, Berger spheres and any compact quotient of a three-dimensional Lie Group. The study of special surfaces (minimal, constant mean curvature, totally umbilic) in homogeneous spaces is a very active area of research: for instance let us mention the recent monograph of Meeks-Perez [26], the recent paper on CMC spheres in homogeneous three-spheres by Meeks-Mira-Perez-Ros [27] and the classification of totally umbilical surfaces in homogeneous three-spaces by Manzano-Souam [24].

Let us stress that most of the results in this setting are for genus 0 surfaces and for second order problems, so the originality of our result lies in both exploring higher genus surfaces and higher order problems (recall that the Willmore equation is of fourth order, while minimal,CMC, and totally umbilical surface equations are of second order).

Before passing to describe the organization of the paper let us mention that our techniques can be adapted to handle also some non compact framework. Indeed, as (remarkable) example, we prove the existence of toroidal area-constrained critical points of the Hawking mass in the Schwarzschild space.

Recall that the Schwarzschild metric of mass $m>0$ is given by $\left(\mathbb{R}^{3} \backslash\{0\}, g_{S c h}\right)$ where $g_{i j}(x)=(1+$ $\left.\frac{m}{2 r}\right)^{4} \delta_{i j}$, where of course $r=\sqrt{\left(x^{1}\right)^{2}+\left(x^{2}\right)^{2}+\left(x^{3}\right)^{2}}$. The metric is spherically symmetric, conformally equivalent to the Euclidean one and asymptotically flat, it has zero scalar curvature, and the sphere at $\{r=m / 2\}$ is totally geodesic. In fact, the Schwarzschild metric is symmetric under the mapping $r \mapsto \frac{m^{2}}{4 r}$ and therefore it has two asymptotically flat ends.

In the next remark we recall what is known about minimal and CMC surfaces in Schwarzschild metric (we thank Alessandro Carlotto for a discussion about this point).

Remark 1.5 (Minimal and CMC surfaces in Schwarzschild). - In the Schwarzschild space there are no non-spherical closed minimal surfaces: indeed it is known that if a closed minimal surface $\Sigma$ in the Schwarzschild space is non-spherical then it must be totally geodesic and in particular totally umbilic, but the fact of being totally umbilic is conformally invariant and therefore $\Sigma$ is totally umbilic also in the Euclidean space. But then $\Sigma$ is a Euclidean round sphere which contradicts that $\Sigma$ was not spherical. More precisely, by a maximum principle argument using comparison with CMC slices, it is possible to show that the only embedded closed minimal hypersurface in $\left(\mathbb{R}^{3} \backslash\{0\}, g_{S c h}\right)$ is the horizon $\{r=m / 2\}$, which in fact is totally geodesic.

- Regarding $C M C$ surfaces in $\left(\mathbb{R}^{3} \backslash\{0\}, g_{S c h}\right)$, it was proved by Brendle [8] that the only embedded closed CMC surfaces in the outer Schwarzschild $\left(\mathbb{R}^{3} \backslash B_{m / 2}(0), g_{S c h}\right)$ are the spherical slices $\{r=$ const $\}$ (let us mention that the results of Brendle include a larger class of warped products metrics). The embeddedness assumption is crucial for this classification result, in view of possible phenomena analogous to the Wente tori (which are immersed and CMC) in $\mathbb{R}^{3}$. It is also essential that the closed surfaces do not intersect the horizon $\{r=m / 2\}$. Indeed, solving the isoperimetric problem in $\left(\mathbb{R}^{3} \backslash\{0\}, g_{\text {Sch }}\right)$ for small volumes, it is expected (by perturbative arguments a la Pacard$X u[35]$ ) that the isoperimetric surfaces are spherical surfaces intersecting $\{r=m / 2\}$ (regarding the isoperimetric problem in Schwarzschild see also the Ph.D. thesis of Bray [7] and the recent paper 
by Brendle-Eichmair [9]).

Summarizing, it is known that in $\left(\mathbb{R}^{3} \backslash\{0\}, g_{S c h}\right)$ there are no non-spherical embedded minimal surfaces and it is expected there are no non-spherical embedded CMC surfaces.

In sharp contrast to the aforementioned situation, our next theorem asserts the existence of embedded tori which are critical points of the Hawking mass under area constraint.

Theorem 1.6. Let $\left(\mathbb{R}^{3} \backslash\{0\}, g_{S c h}\right)$, with $g_{i j}(x)=\left(1+\frac{m}{2 r}\right)^{4} \delta_{i j}$, be the Schwarzschild metric of mass $m>0$. Then there exists $\varepsilon_{0}>0$ such that for every $\varepsilon \in\left(0, \varepsilon_{0}\right)$ there exist smooth embedded tori (infinitely-many, by symmetry) in $\left(\mathbb{R}^{3} \backslash\{0\}, g_{S c h}\right)$ which are critical points of the Hawking mass with constrained area equal to $4 \sqrt{2} \pi^{2} \varepsilon^{2}$.

Remark 1.7. With analogous arguments to the proof of Theorem 1.6 one can actually prove existence of smooth embedded tori which are critical points of the Hawking mass with small constrained area in asymptotically locally Euclidean (ALE) scalar flat 3-manifolds (for more details see Remark 5.2). More precisely, the following two conditions are sufficient for our arguments.

1) $(M, g)$ is a complete non compact 3-manifold whose scalar curvature vanishes identically: Sc $\equiv 0$.

2) Fixed some base point $x_{0} \in M$, there exists $r>0$ with the following property: for every $\epsilon>0$ there exists $R_{\epsilon}>0$ such that for any $x \in M \backslash B_{R_{\epsilon}}^{M}\left(x_{0}\right)$ there exists a diffeomorphism $\Psi: B_{r}^{\mathbb{R}^{3}}(0) \rightarrow B_{r}^{M}(x)$ satisfying $\left\|\delta_{i j}-\left(\Psi^{*} g\right)_{i j}\right\|_{C^{2}\left(B_{r}^{\mathbb{R}^{3}}(0)\right)} \leq \epsilon$.

Notice that condition 1) is equivalent to the constrained Einstein equations in the vacuum case, and 2) is a mild uniform control of the local geometry of $M$ together with a mild asymptotic condition.

Now we pass to outline the structure of the paper and the main ideas of the construction: a LyapunovSchmidt reduction. Roughly, such technique can be summarized as follows (for a systematic presentation with examples see the monograph of Ambrosetti and the second author [3]; the original technique is due to Lyapunov-Schmidt but the variational implementation we are going to describe is inspired by the work of Ambrosetti-Badiale [1]-[2]): one is interested in finding critical points of a real valued functional $I_{\varepsilon}$ from infinite dimensional space $X$, knowing that for $\varepsilon>0$ small enough there exists a finite dimensional manifold $Z_{\varepsilon} \subset X$ made of almost critical points of $I_{\varepsilon}$ (in the sense that the differential $I_{\varepsilon}^{\prime}$ is small enough on $Z_{\varepsilon}$ in a suitable sense).

If one also knows that the second differential $I_{\varepsilon}^{\prime \prime}$ is non degenerate on $Z_{\varepsilon}$, or more precisely $I_{\varepsilon}^{\prime \prime}$ restricted to the orthogonal complement of the tangent space to $Z_{\varepsilon}$ is non degenerate (here orthogonal complement must be considered just formally as a motivation, but this is rigorous if $X$ is a Hilbert space), then one can solve an auxiliary equation (given by the projection of the equation $I_{\varepsilon}^{\prime}=0$ onto the orthogonal complement of $T Z_{\varepsilon}$ ) and reduce the problem of finding critical points of the functional $I_{\varepsilon}: X \rightarrow \mathbb{R}$ to finding critical points of a suitable functional $\Phi_{\varepsilon}: Z_{\varepsilon} \rightarrow \mathbb{R}$; of course the advantage being the reduction of the problem to studying a function of finitely many variables.

In our case the functional $I_{\varepsilon}$ is of course the Willmore energy $W$ defined in (1) and $X$ is the space of smooth immersions (with area constraint $\varepsilon$ ) from the Clifford torus into the Riemannian manifold $(M, g)$. Observe that, by the conformal invariance of the Willmore functional in $\mathbb{R}^{3}$, the family of (images under Möbius transformations of) Clifford tori in $\mathbb{R}^{3}$ form a non compact critical manifold for the Euclidean Willmore functional; moreover from the classical paper of Weiner 43 it is known that the second variation of $W$ is non degenerate on this critical manifold and by the recent gap-theorem proved by Nguyen and the third author [30] this critical manifold is isolated in energy from the next Willmore torus. In Section 2 we describe in some detail the Möbius maps which preserve the area of the Clifford torus, as well as Weiner's result.

Since at small scales a Riemannian metric approaches the Euclidean one, it is natural to expect that the images of small Clifford tori via exponential map form a manifold of almost critical points of $W$ in the above sense. This will be proved, with quantitative estimates, in Section 3.1. After that, the finitedimensional reduction of the problem will be carried out: in Proposition 3.5 for every (exponentiated) torus we will construct a perturbation which will solve our problem up to some further Lagrange multipliers given by the Jacobi fields of translations, rotations and Möbius inversions. The abstract construction 
in Section 3 is rather standard, nevertheless the main obstacle here is due to the fact that the action of the Möbius group on the Clifford torus is non-compact.

To overcome this issue we incorporate in the abstract construction the variational structure of the problem. We will compare the Willmore energy of (exponentiated) symmetric VS degenerating tori. These expansions are worked-out in Section 4 (see in particular Proposition 4.1 and Proposition 4.6 respectively), where the effect of curvature is taken into account. Degenerate small tori look like geodesic spheres with small handles, so for these we check that the scalar curvature of $M$ plays the main role in the expansion (compare to the results in [17] and [28]). For symmetric tori instead we observe an effect due to a combination of the scalar curvature and the sectional curvature of the plane of symmetry of the torus (which can be expressed in terms of the Ricci tensor in the axial direction of the torus), see Remark 4.4 These expansions are probably the main contribution of the present work; we believe that they might play a role in further developments of the topic, especially in ruling-out possible degeneracy phenomena under global (non-perturbative) variational approaches to the problem, as it has already happened for the case of Willmore spheres.

In Section 5.1 we will use the assumptions in Theorem 1.1 for ruling out Möbius degeneration by direct energy comparison, which would be more costly. We will consider a sequence of compact sets invading the family of Möbius inverted tori and we will show that the Willmore energy in the interior is strictly lower (or higher) than the energy on their boundary. This will prove the existence of a critical point despite the non-compactness of the problem. Finally, some examples to the applicability of Theorem 1.1 are provided.

\section{Acknowledgements}

The first author was supported by JSPS Research Fellowships 24-2259. The second author is supported by the project Geometric Variational Problems from Scuola Normale Superiore and by MIUR Bando PRIN 2015 2015KB9WPT 001 . He is also member of GNAMPA as part of INdAM. The third author acknowledges the support of the ETH fellowship. This work was done while the first author visited the University of Warwick and SISSA: he would like to express his gratitude for the hospitality.

\section{Preliminaries}

In this section, we discuss the Möbius inversions preserving the area of the Clifford Torus $\mathbb{T}$. Moreover, we collect some properties of the Willmore functional. Hereafter, we use the notations $\langle\cdot, \cdot\rangle$ and $g_{0}(\cdot, \cdot)$ for the Euclidean metric.

\subsection{Inverted tori with fixed area}

This subsection is devoted to the analysis of the Möbius inversions of $\mathbb{T}$ which preserve the area of the torus. Through this paper, we parametrize the Clifford torus $\mathbb{T}$ by

$$
\mathbb{T}:=\{X(\varphi, \theta): \varphi, \theta \in[0,2 \pi]\}
$$

where

$$
X(\varphi, \theta):=((\sqrt{2}+\cos \varphi) \cos \theta,(\sqrt{2}+\cos \varphi) \sin \theta, \sin \varphi) .
$$

By this parametrization, the area element $d \sigma_{\mathbb{T}}$ is given by

$$
d \sigma_{\mathbb{T}}=(\sqrt{2}+\cos \varphi) d \varphi d \theta .
$$

For $x_{0} \in \mathbb{R}^{3}$ and $\eta>0$, the Möbius inversion with respect to $\partial B_{\eta}\left(x_{0}\right)$ is defined by

$$
\Phi_{x_{0}, \eta}(x):=\frac{\eta^{2}}{\left|x-x_{0}\right|^{2}}\left(x-x_{0}\right)+x_{0} .
$$


For any smooth compact surface $\Sigma \subset \mathbb{R}^{3} \backslash\left\{x_{0}\right\}$, we set $\bar{\Sigma}:=\Phi_{x_{0}, \eta}(\Sigma)$ and we denote the volume elements of $\Sigma$ and $\bar{\Sigma}$ by $d \sigma_{\Sigma}$ and $d \sigma_{\bar{\Sigma}}$ respectively. By the conformality of $\Phi_{x_{0}, \eta}$ it turns out that

$$
d \sigma_{\bar{\Sigma}}=\frac{\eta^{4}}{\left|x-x_{0}\right|^{4}} d \sigma_{\Sigma}
$$

We are interested in two opposite limit behaviours of the Möbius inversions applied to $\mathbb{T}$, namely for large and small inversion radii. First of all, we observe the existence and basic properties of the Möbius inversions preserving the area of $\mathbb{T}$.

Lemma 2.1. Let $\mathbf{e}_{x}:=(1,0,0)$. Then for any $\eta>0$, there exists a unique $\xi_{\eta}>\sqrt{2}+1$ such that

$$
\left|\Phi_{-\xi_{\eta} \mathbf{e}_{x}, \eta}(\mathbb{T})\right|_{g_{0}}=4 \sqrt{2} \pi^{2}=|\mathbb{T}|_{g_{0}}
$$

where $|A|_{g_{0}}$ denotes the area of a surface $A$ in the Euclidean space. Moreover, the map $\eta \mapsto \xi_{\eta}$ is strictly increasing in $(\sqrt{2}+1, \infty)$ and smooth. Finally, one observes that

$$
\lim _{\eta \rightarrow 0} \xi_{\eta}=\sqrt{2}+1, \quad \lim _{\eta \rightarrow \infty} \xi_{\eta}=\infty .
$$

Proof. First we notice that $\mathbb{T} \subset \mathbb{R}^{3} \backslash\left\{\xi \mathbf{e}_{x}\right\}$ for each $\xi>\sqrt{2}+1$, so $\Phi_{-\xi \mathbf{e}_{x}, \eta}(\mathbb{T})$ is well-defined for every $\xi>\sqrt{2}+1$ and $\eta>0$. Thus by (6) and (8), we observe

$$
A(\xi, \eta):=\left|\Phi_{-\xi \mathbf{e}_{x}, \eta}(\mathbb{T})\right|_{g_{0}}=\eta^{4} \int_{0}^{2 \pi} \int_{0}^{2 \pi} \frac{\sqrt{2}+\cos \varphi}{\left|X(\varphi, \theta)+\xi \mathbf{e}_{x}\right|^{4}} d \varphi d \theta
$$

Moreover, it is easily seen that

$$
A \in C^{\infty}((\sqrt{2}+1, \infty) \times(0, \infty)), \quad \frac{\partial A}{\partial \eta}(\xi, \eta)>0>\frac{\partial A}{\partial \xi}(\xi, \eta) \quad \text { for all }(\xi, \eta) \in(\sqrt{2}+1, \infty) \times(0, \infty) .
$$

Thus from

$$
\lim _{\eta \rightarrow 0} A(\xi, \eta)=0, \quad \lim _{\eta \rightarrow \infty} A(\xi, \eta)=\infty,
$$

we may find a unique $\xi_{\eta}>\sqrt{2}+1$ so that $A\left(\xi_{\eta}, \eta\right)=4 \sqrt{2} \pi^{2}$. By the implicit function theorem, $\xi_{\eta}$ is smooth and strictly increasing from (9). Finally, it is obvious to see that

$$
\lim _{\eta \rightarrow 0} \xi_{\eta}=\sqrt{2}+1, \quad \lim _{\eta \rightarrow \infty} \xi_{\eta}=\infty
$$

which completes the proof.

First we observe the behaviour of $\xi_{\eta}$ and $\Phi_{-\xi_{\eta}, \eta}$ as $\eta \rightarrow \infty$.

Lemma 2.2. As $\eta \rightarrow \infty$, there holds

$$
\frac{\xi_{\eta}}{\eta}=1+O\left(\eta^{-2}\right), \quad \Phi_{-\xi_{\eta} \mathbf{e}_{x}, \eta}(x) \rightarrow x-2\left\langle x, \mathbf{e}_{x}\right\rangle \mathbf{e}_{x} \quad \text { in } C_{\mathrm{loc}}^{\infty}\left(\mathbb{R}^{3}\right) .
$$

Remark 2.3. The map $x \mapsto x-2\left\langle x, \mathbf{e}_{x}\right\rangle \mathbf{e}_{x}$ is the reflection with respect to the plane $\left[x^{1}=0\right]:=$ $\left\{\left(0, x^{2}, x^{3}\right): x^{2}, x^{3} \in \mathbb{R}\right\}$.

Proof of Lemma 2.2. First, we prove $\xi_{\eta} \eta^{-1}=1+O\left(\eta^{-2}\right)$. Since $\xi_{\eta} \rightarrow \infty$ as $\eta \rightarrow \infty$, one has

$$
\left|\xi_{\eta}^{-1} X+\mathbf{e}_{x}\right|^{4}=1+4 \xi_{\eta}^{-1}(\sqrt{2}+\cos \varphi) \cos \theta+O\left(\xi_{\eta}^{-2}\right) .
$$


Thus by a Taylor expansion we obtain

$$
\begin{aligned}
4 \sqrt{2} \pi^{2} & =\frac{\eta^{4}}{\xi_{\eta}^{4}} \int_{0}^{2 \pi} \int_{0}^{2 \pi} \frac{\sqrt{2}+\cos \varphi}{\left|\xi_{\eta}^{-1} X+\mathbf{e}_{x}\right|^{4}} d \theta d \varphi \\
& =\frac{\eta^{4}}{\xi_{\eta}^{4}} \int_{0}^{2 \pi} \int_{0}^{2 \pi}(\sqrt{2}+\cos \varphi)\left\{1-4 \xi_{\eta}^{-1}(\sqrt{2}+\cos \varphi) \cos \theta+O\left(\xi_{\eta}^{-2}\right)\right\} d \theta d \varphi .
\end{aligned}
$$

Noting that

$$
\int_{0}^{2 \pi} \int_{0}^{2 \pi}(\sqrt{2}+\cos \varphi)^{2} \cos \theta d \theta d \varphi=0
$$

we have

$$
4 \sqrt{2} \pi^{2}=\frac{\eta^{4}}{\xi_{\eta}^{4}}\left\{4 \sqrt{2} \pi^{2}+O\left(\xi_{\eta}^{-2}\right)\right\} .
$$

Hence, $\xi_{\eta} \eta^{-1} \rightarrow 1$ as $\eta \rightarrow \infty$ and $\xi_{\eta} \eta^{-1}=1+O\left(\eta^{-2}\right)$.

For the latter claim, we first remark that

$$
\Phi_{-\xi_{\eta} \mathbf{e}_{x}, \eta}(x)=\frac{\eta^{2}}{\left|x+\xi_{\eta} \mathbf{e}_{x}\right|^{2}} x+\xi_{\eta}\left(\frac{\eta^{2}}{\left|x+\xi_{\eta} \mathbf{e}_{x}\right|^{2}}-1\right) \mathbf{e}_{x}
$$

and

$$
\frac{\eta^{2}}{\left|x+\xi_{\eta} \mathbf{e}_{x}\right|^{2}}=\left(\frac{\eta}{\xi_{\eta}}\right)^{2} \frac{1}{\left|\mathbf{e}_{x}+\xi_{\eta}^{-1} x\right|^{2}}=\left(1+O\left(\eta^{-2}\right)\right)\left(1-2 \xi_{\eta}^{-1}\left\langle x, \mathbf{e}_{x}\right\rangle \mathbf{e}_{x}+O\left(\eta^{-2}\right)\right) .
$$

Hence,

$$
\Phi_{-\xi_{\eta} \mathbf{e}_{x}, \eta}(x)=\left(1+O\left(\eta^{-1}\right)\right) x-2\left\langle x, \mathbf{e}_{x}\right\rangle \mathbf{e}_{x}+O\left(\eta^{-1}\right),
$$

and the claim follows.

Next, we consider the behaviour of the Möbius inversions as $\eta \rightarrow 0$. For this purpose, it is convenient to fix a symmetric point of $\Phi_{-\xi_{\eta} \mathbf{e}_{x}, \eta}$. Since either the translations or reflections do not affect areas, from Lemma 2.1 there exists $\tilde{\xi}_{\eta}>0$ for every $\eta>0$ so that

$$
\left|\Phi_{0, \eta}\left(\mathbb{T}_{\tilde{\xi}_{\eta}}\right)\right|_{g_{0}}=4 \sqrt{2} \pi^{2} \quad \text { where } \quad \mathbb{T}_{\tilde{\xi}_{\eta}}:=\mathbb{T}-\left(\sqrt{2}+1+\tilde{\xi}_{\eta}\right) \mathbf{e}_{x}
$$

Furthermore, $\tilde{\xi}_{\eta}$ is smooth, strictly increasing in $(0, \infty)$ and satisfies

$$
\lim _{\eta \rightarrow 0} \tilde{\xi}_{\eta}=0 .
$$

Next, we remark the following two properties of the Möbius inversions:

(i) $\Phi_{0, \eta}(x)=\Phi_{0,1}\left(\eta^{-2} x\right)$ for every $\eta>0$ and $x \in \mathbb{R}^{3} \backslash\{0\}$.

(ii) For any $c_{0} \in \mathbb{R} \backslash\{0\}, \Phi_{0,1}$ maps the locus $\left\{x^{1}=c_{0}\right\}$ into a sphere of radius $1 /\left(2\left|c_{0}\right|\right)$ centred at $\left(c_{0} /\left(2\left|c_{0}\right|^{2}\right)\right) \mathbf{e}_{x}$.

Then we define the following functions:

$$
\begin{aligned}
Y(\varphi, \theta, \eta) & :=X(\varphi, \theta)-\left(\sqrt{2}+1+\tilde{\xi}_{\eta}\right) \mathbf{e}_{x} \\
Z(\bar{\varphi}, \bar{\theta}, \eta) & :=\Phi_{0, \eta}\left(Y\left(\eta^{2} \bar{\varphi}, \eta^{2} \bar{\theta}, \eta\right)\right)=\Phi_{0,1}\left(\eta^{-2} Y\left(\eta^{2} \bar{\varphi}, \eta^{2} \bar{\theta}, \eta\right)\right)
\end{aligned}
$$

for $(\bar{\varphi}, \bar{\theta}) \in \mathbb{R}^{2}$. We shall show the following result: 
Lemma 2.4. (i) $\lim _{\eta \rightarrow 0} \eta^{2} / \tilde{\xi}_{\eta}=2 \sqrt[4]{2 \pi^{2}}$. Furthermore, $\eta^{2} / \tilde{\xi}_{\eta}=2 \sqrt[4]{2 \pi^{2}}+O\left(\eta^{2}\right)$.

(ii) $\Phi_{0, \eta}\left(\mathbb{T}_{\tilde{\xi}_{\eta}}\right)$ converges to the sphere with radius $\sqrt[4]{2 \pi^{2}}$ centred at $-\sqrt[4]{2 \pi^{2}} \mathbf{e}_{x}$ in the following sense: for any $R>0$ and $k \in \mathbb{N}$, if $\eta \leq 1 / R^{4}$, then

$$
\left\|Z(\cdot, \cdot, \eta)-Z_{0}\right\|_{C^{k}\left([-R, R]^{2}\right)} \leq C_{k} \eta^{3 / 2}
$$

as $\eta \rightarrow 0$, where $C_{k}$ depends only on $k$ and $Z_{0}$ is defined by

$$
Z_{0}(\bar{\varphi}, \bar{\theta}):=\Phi_{0,1}\left(-\frac{1}{2 \sqrt[4]{2 \pi^{2}}} \mathbf{e}_{x}+(\sqrt{2}+1) \bar{\theta} \mathbf{e}_{y}+\bar{\varphi} \mathbf{e}_{z}\right), \quad \mathbf{e}_{y}:=(0,1,0), \quad \mathbf{e}_{z}:=(0,0,1) .
$$

Proof. For assertion (i), it is enough to prove

$$
\frac{\eta^{4}}{\tilde{\xi}_{\eta}^{2}}=4 \sqrt{2} \pi+O\left(\eta^{2}\right)
$$

as $\eta \rightarrow 0$. To this aim, for each $\eta \in(0,1]$, we set

$$
I_{\eta}:=\left\{(\varphi, \theta) \in[-\pi, \pi]^{2}: \varphi^{2}+(\sqrt{2}+1)^{2} \theta^{2} \leq \eta^{2}\right\}, \quad J_{\eta}:=[-\pi, \pi]^{2} \backslash I_{\eta} .
$$

Recall that $\Phi_{0, \eta}\left(\mathbb{T}_{\tilde{\xi}_{\eta}}\right)$ has the fixed area $4 \sqrt{2} \pi^{2}$ :

$$
4 \sqrt{2} \pi^{2}=\eta^{4} \int_{-\pi}^{\pi} \int_{-\pi}^{\pi} \frac{\sqrt{2}+\cos \varphi}{|Y(\varphi, \theta, \eta)|^{4}} d \varphi d \theta=\eta^{4}\left(\int_{J_{\eta}}+\int_{I_{\eta}}\right) \frac{\sqrt{2}+\cos \varphi}{|Y(\varphi, \theta, \eta)|^{4}} d \varphi d \theta=: \tilde{I}_{1}+\tilde{I}_{2}
$$

where we used $[-\pi, \pi]^{2}$ instead of $[0,2 \pi]^{2}$.

First we shall show $\tilde{I}_{1}=O\left(\eta^{2}\right)$. By a Taylor expansion of $Y$ around $(0,0)$, we have

$$
\left|Y\left(\varphi, \theta, \xi_{\eta}\right)\right|^{2}=\varphi^{2}+(\sqrt{2}+1)^{2} \theta^{2}+\tilde{\xi}_{\eta}^{2}+\left\{(\sqrt{2}+1) \theta^{2}+\varphi^{2}\right\} \tilde{\xi}_{\eta}+O\left(\varphi^{4}+\theta^{4}\right) .
$$

Thus we may find $C_{0}>0$, which is independent of $\eta \in(0,1]$, so that

$$
C_{0}\left(\varphi^{2}+(\sqrt{2}+1)^{2} \theta^{2}\right) \leq|Y(\varphi, \theta, \eta)|^{2} \quad \text { for all }(\varphi, \theta) \in J_{\eta} \quad \text { and } \quad \eta \in(0,1] .
$$

Hence, using the change of variables $(\varphi, \theta)=\left(r \cos \Theta,(\sqrt{2}+1)^{-1} r \sin \Theta\right)$ and noting that $J_{\eta} \subset\{(r, \Theta) \in$ $[\eta, 4 \pi] \times[0,2 \pi]\}$, we obtain

$$
\int_{J_{\eta}} \frac{\sqrt{2}+\cos \varphi}{|Y(\varphi, \theta, \eta)|^{4}} d \varphi d \theta \leq C_{1} \int_{J_{\eta}} \frac{d \varphi d \theta}{\left(\varphi^{2}+(\sqrt{2}+1)^{2} \theta^{2}\right)^{2}}=\frac{C_{1}}{\sqrt{2}+1} \int_{\eta}^{4 \pi} \int_{0}^{2 \pi} \frac{r}{r^{4}} d r d \Theta \leq C_{2} \eta^{-2} .
$$

Thus multiplying the above inequality by $\eta^{4}$ we get $\tilde{I}_{1}=O\left(\eta^{2}\right)$.

Next, we examine $\tilde{I}_{2}$. Recalling (12), we have

$$
\left\{1-O\left(\eta^{2}\right)-\tilde{\xi}_{\eta}\right\}\left(\varphi^{2}+(\sqrt{2}+1)^{2} \theta^{2}\right)+\tilde{\xi}_{\eta}^{2} \leq|Y(\varphi, \theta, \eta)|^{2} \leq\left\{1+O\left(\eta^{2}\right)+\tilde{\xi}_{\eta}\right\}\left\{\varphi^{2}+(\sqrt{2}+1)^{2} \theta^{2}\right\}+\tilde{\xi}_{\eta}^{2}
$$

for every $(\varphi, \theta) \in I_{\eta}$. Now we compute

$$
\eta^{4} \int_{I_{\eta}} \frac{(\sqrt{2}+1) d \varphi d \theta}{\left[\left\{1+O\left(\eta^{2}\right)+\tilde{\xi}_{\eta}\right\}\left\{\varphi^{2}+(\sqrt{2}+1)^{2} \theta^{2}\right\}+\tilde{\xi}_{\eta}^{2}\right]^{2}} .
$$


Using the same change of variables as above and noting that $\tilde{\xi}_{\eta} \rightarrow 0$ as $\eta \rightarrow 0$, we have

$$
\begin{aligned}
& \eta^{4} \int_{I_{\eta}} \frac{(\sqrt{2}+1) d \varphi d \theta}{\left[\left\{1+O\left(\eta^{2}\right)+\tilde{\xi}_{\eta}\right\}\left\{\varphi^{2}+(\sqrt{2}+1)^{2} \theta^{2}\right\}+\tilde{\xi}_{\eta}^{2}\right]^{2}} \\
= & 2 \pi \eta^{4} \int_{0}^{\eta} \frac{r d r}{\left[\left\{1+O\left(\eta^{2}\right)+\tilde{\xi}_{\eta}\right\} r^{2}+\tilde{\xi}_{\eta}^{2}\right]^{2}} \\
= & \frac{\pi \eta^{4}}{1+O\left(\eta^{2}\right)+\tilde{\xi}_{\eta}}\left(\frac{1}{\tilde{\xi}_{\eta}^{2}}-\frac{1}{\eta^{2}\left(1+\tilde{\xi}_{\eta}+O\left(\eta^{2}\right)\right)+\tilde{\xi}_{\eta}^{2}}\right) \\
= & \left\{1-\tilde{\xi}_{\eta}-O\left(\eta^{2}\right)-O\left(\tilde{\xi}_{\eta}^{2}\right)\right\} \pi\left(\frac{\eta^{4}}{\tilde{\xi}_{\eta}^{2}}+O\left(\eta^{2}\right)\right) .
\end{aligned}
$$

Combining (11), (13) and (14) with $\tilde{I}_{1}=O\left(\eta^{2}\right)$ and $\sqrt{2}+\cos \varphi=\sqrt{2}+1+O\left(\eta^{2}\right)$ in $I_{\eta}$, one may observe that

$$
\left\{1-\tilde{\xi}_{\eta}+O\left(\eta^{2}\right)+O\left(\tilde{\xi}_{\eta}^{2}\right)\right\} \frac{\eta^{4}}{\tilde{\xi}_{\eta}^{2}}=4 \sqrt{2} \pi+O\left(\eta^{2}\right)
$$

Since $\tilde{\xi}_{\eta} \rightarrow 0$ as $\eta \rightarrow 0$, we have $\eta^{4} / \tilde{\xi}_{\eta}^{2} \rightarrow 4 \sqrt{2} \pi$. Hence, combining (15), we get (10).

For assertion (ii), due to a Taylor expansion, we first remark that

$$
\cos \theta=1+R_{c}(\theta), \quad \sin \theta=\theta+R_{s}(\theta)
$$

where

$$
\left|\frac{d^{k}}{d \theta^{k}} R_{c}(\theta)\right| \leq C_{k}|\theta|^{(2-k)_{+}}, \quad\left|\frac{d^{k}}{d \theta^{k}} R_{s}(\theta)\right| \leq C_{k}|\theta|^{(3-k)_{+}}
$$

for all $k \in \mathbb{N}$ and $|\theta| \leq 1$ where $a_{+}:=\max \{0, a\}$. Using these expansions, we obtain

$$
\eta^{-2} Y\left(\eta^{2} \bar{\varphi}, \eta^{2} \bar{\theta}, \eta\right)=\left(-\eta^{-2} \tilde{\xi}_{\eta}+R_{Y, \eta, 1}(\bar{\varphi}, \bar{\theta}), \quad(\sqrt{2}+1) \bar{\theta}+R_{Y, \eta, 2}(\bar{\varphi}, \bar{\theta}), \quad \bar{\varphi}+R_{Y, \eta, 3}(\bar{\varphi}, \bar{\theta})\right)
$$

where

$$
\begin{aligned}
& R_{Y, \eta, 1}(\bar{\varphi}, \bar{\theta}):=(\sqrt{2}+1) \eta^{-2} R_{c}\left(\eta^{2} \bar{\theta}\right)+\eta^{-2} R_{c}\left(\eta^{2} \bar{\varphi}\right)+\eta^{-2} R_{c}\left(\eta^{2} \bar{\varphi}\right) R_{c}\left(\eta^{2} \bar{\theta}\right), \\
& R_{Y, \eta, 2}(\bar{\varphi}, \bar{\theta}):=(\sqrt{2}+1) \eta^{-2} R_{s}\left(\eta^{2} \bar{\theta}\right)+\bar{\theta} R_{c}\left(\eta^{2} \bar{\varphi}\right)+\eta^{-2} R_{c}\left(\eta^{2} \bar{\varphi}\right) R_{s}\left(\eta^{2} \bar{\theta}\right) \\
& R_{Y, \eta, 3}(\bar{\varphi}, \bar{\theta}):=\eta^{-2} R_{s}\left(\eta^{2} \bar{\varphi}\right) .
\end{aligned}
$$

By (16), it is easy to verify that

$$
\left\|R_{Y, \eta, 1}\right\|_{C^{k}\left([-R, R]^{2}\right)} \leq C_{k} \eta^{3 / 2}, \quad\left\|R_{Y, \eta, 2}\right\|_{C^{k}\left([-R, R]^{2}\right)} \leq C_{k} \eta^{3}, \quad\left\|R_{Y, \eta, 3}\right\|_{C^{k}\left([-R, R]^{2}\right)} \leq C_{k} \eta^{3}
$$

for all $k \in \mathbb{N}$ provided $\eta \leq 1 / R^{4}$. Noting the assertion (i) and

$$
\left\|\Phi_{0,1}\right\|_{C^{k}\left(\mathbb{R}^{3} \backslash B_{r}(0)\right)} \leq C_{r}
$$

for each $r>0$, the assertion (ii) holds.

By Lemmas 2.12.2, 2.4 and Remark 2.3, composing the inversions in Lemma2.1 with suitable translations, rotations and reflections, one can obtain a smooth two-dimensional family of transformations of the Clifford torus $\mathbb{T}$ which contains the identity and which degenerates to spheres centred on the $x y$-plane 
and passing through the origin of $\mathbb{R}^{3}$. Even though the identity map corresponds to choosing $\eta=\infty$ in Lemma 2.1] the smoothness of the entire family (near the identity map) can be checked noticing that the above inversions can also be obtained composing Möbius transformations in $S^{3}$ (including the identity map) and stereographic projections from $S^{3}$ to $\mathbb{R}^{3}$. To summarize the discussion, we state the following proposition.

Proposition 2.5. There exists a smooth family of conformal immersions $T_{\omega}$ of $\mathbb{T}$ into $\mathbb{R}^{3}$, parametrized by $\omega \in \mathbb{D}, \mathbb{D}$ being the unit disk of $\mathbb{R}^{2}$, which preserve the area of $\mathbb{T}$ and for which the following hold

a) $T_{0}=\mathrm{Id}$;

b) for $\omega \neq 0, T_{\omega}$ is an inversion with respect to a sphere centred at a point in $\mathbb{R}^{3}$ aligned to $\omega$ (viewed as an element of $\mathbb{R}^{3}$ );

c) as $|\omega|$ approaches $1, T_{\omega}(\mathbb{T})$ degenerates to a sphere of radius $\sqrt[4]{2 \pi^{2}}$ centred at $\sqrt[4]{2 \pi^{2}} \frac{\omega}{|\omega|}$.

In what follows, we use the symbol $\mathbb{T}_{\omega}$ for $T_{\omega}(\mathbb{T})$.

Remark 2.6. If we wish to rotate in $\mathbb{R}^{3}$ the images $\mathbb{T}_{\omega}$, the resulting surfaces can be parametrized by the family of tangent vectors to $\mathbb{R P}^{2}$ with length less than 1 .

\subsection{Basic properties of the Willmore functional}

In this subsection, we state basic properties of the Willmore functional as well as the non-degeneracy condition of $\mathbb{T}$. First of all, we begin with a basic property of the Willmore functional $W_{0}$ in the Euclidean space for immersions $i: \Sigma \rightarrow \mathbb{R}^{3}$

$$
W_{0}(i(\Sigma))=\int_{\Sigma} H^{2} d \sigma,
$$

namely its conformal invariance.

Proposition 2.7. Let $\Sigma$ be a compact closed surface of class $C^{2}$ and let $i: \Sigma \rightarrow \mathbb{R}^{3}$ be an immersion. Then, if $\lambda>0$ and if $\Phi_{x_{0}, \eta}$ is as in (7), one has the invariance properties

$$
\text { j) } \left.\quad W_{0}(\lambda i(\Sigma))=W_{0}(i(\Sigma)) \quad \text { and } \quad j j\right) \quad W_{0}\left(\left(\Phi_{x_{0}, \eta} \circ i\right)(\Sigma)\right)=W_{0}(i(\Sigma)) \quad \text { provided } x_{0} \notin i(\Sigma) \text {. }
$$

We also recall the first and second variation formulas for the Willmore functional

$$
W(i(\Sigma))=\int_{\Sigma} H^{2} d \sigma
$$

in a general setting, namely for a surface immersed in a three-dimensional manifold $M$. We perturb the surface through a variation with normal speed $\varphi n$, where $n$ stands for the unit outer normal to the image of $\Sigma$. We denote the perturbed surfaces by $F(t, p)(t \in(-\varepsilon, \varepsilon), p \in \Sigma)$. Calling $n=n(t, p)$ the outward pointing unit normal to the immersion $F(t, \cdot)$ we denote with $\varphi:=g\left(n, \partial_{t} F\right)$ the normal velocity, we also let $\bar{g}_{i j}$ be the first fundamental form of $i(\Sigma)$ and by $d \sigma$ the induced area element.

We denote by Riem the Riemann curvature tensor of $M$ at $P$, by Ric the Ricci tensor, by Sc the scalar curvature, by $A$ the second fundamental form of $i(\Sigma)$, by $\AA=A-\frac{1}{2} H \bar{g}$ the traceless part of $A$, by $\Delta$ the Laplace-Beltrami operator on $(\Sigma, \bar{g})$ and we define the elliptic, self-adjoint operator

$$
L \varphi:=-\Delta \varphi-\varphi\left(|A|^{2}+\operatorname{Ric}(n, n)\right) .
$$

Here, for Riem and $A$, we use the following convention:

$$
\operatorname{Riem}(X, Y) Z=\nabla_{X} \nabla_{Y} Z-\nabla_{Y} \nabla_{X} Z-\nabla_{[X, Y]} Z, \quad A(X, Y)=g\left(\nabla_{X} n, Y\right) .
$$

We also define the one-form $\varpi$ to be the tangent component of the one-form $\operatorname{Ric}(n, \cdot)$ in $M$, namely

$$
\varpi=\operatorname{Ric}(n, \cdot)^{t}
$$


and the $(2,0)$ tensor $T$ by

$$
T_{i j}=\operatorname{Riem}\left(\partial_{i}, n, n, \partial_{j}\right)=\operatorname{Ric}_{i j}+G(n, n) \bar{g}_{i j},
$$

where $G=\operatorname{Ric}-\frac{1}{2} \mathrm{Sc} g$ is the Einstein tensor of $M$.

We have then the following formulas, see Section 3 in [19].

Proposition 2.8. With the above notation we have the formulas

$$
W^{\prime}(i(\Sigma))[\varphi]=\int_{\Sigma}\left(L H+\frac{1}{2} H^{3}\right) \varphi d \sigma
$$

and

$$
\begin{aligned}
W^{\prime \prime}(i(\Sigma))[\varphi, \varphi]= & 2 \int_{\Sigma}\left[(L \varphi)^{2}+\frac{1}{2} H^{2}|\nabla \varphi|^{2}-2 \AA(\nabla \varphi, \nabla \varphi)\right] d \sigma \\
& +2 \int_{\Sigma} \varphi^{2}\left(|\nabla H|_{g}^{2}+2 \varpi(\nabla H)+H \Delta H+2 g\left(\nabla^{2} H, \AA\right)+2 H^{2}|\AA|_{g}^{2}\right. \\
& \left.+2 H g(\AA, T)-H g(\nabla n \operatorname{Ric})(n, n)-\frac{1}{2} H^{2}|A|_{g}^{2}-\frac{1}{2} H^{2} \operatorname{Ric}(n, n)\right) d \sigma \\
& +\int_{\Sigma}\left(L H+\frac{1}{2} H^{3}\right)\left(\left.\frac{\partial \varphi}{\partial t}\right|_{t=0}+H \varphi^{2}\right) d \sigma \\
= & 2 \int_{\Sigma} \varphi \tilde{L} \varphi d \sigma+\int_{\Sigma}\left(L H+\frac{1}{2} H^{3}\right)\left(\left.\frac{\partial \varphi}{\partial t}\right|_{t=0}+H \varphi^{2}\right) d \sigma
\end{aligned}
$$

where the fourth-order operator $\tilde{L}$ is defined by

$$
\begin{aligned}
\tilde{L} \varphi= & L L \varphi+\frac{1}{2} H^{2} L \varphi+2 H g\left(\AA, \nabla^{2} \varphi\right)+2 H \varpi(\nabla \varphi)+2 \AA(\nabla \varphi, \nabla H) \\
& +\varphi\left(|\nabla H|_{g}^{2}+2 \varpi(\nabla H)+H \Delta H+2 g\left(\nabla^{2} H, \AA\right)+2 H^{2}|\AA|_{g}^{2}+2 H g(\AA, T)-H\left(\nabla_{n} \operatorname{Ric}\right)(n, n)\right) .
\end{aligned}
$$

Remark 2.9. Let us identify $W^{\prime}(i(\Sigma))$ with the function $L H+H^{3} / 2$ on $\Sigma$. Then the derivative of this function with respect to the above perturbation is given by $\tilde{L} \varphi$.

In particular, when $\Sigma$ is embedded in the Euclidean space $\mathbb{R}^{3}$ we obtain the following result, see also [43].

Corollary 2.10.

$$
W_{0}^{\prime}(i(\Sigma))[\varphi]=\int_{\Sigma}\left(L_{0} H+\frac{1}{2} H^{3}\right) \varphi d \sigma ; \quad L_{0} \psi=-\Delta \psi-\psi|A|^{2},
$$

and

$$
\begin{aligned}
W_{0}^{\prime \prime}(i(\Sigma))[\varphi, \varphi]= & 2 \int_{\Sigma}\left[\left(L_{0} \varphi\right)^{2}+\frac{1}{2} H^{2}|\nabla \varphi|^{2}-2 \AA(\nabla \varphi, \nabla \varphi)\right] d \sigma \\
& +2 \int_{\Sigma} \varphi^{2}\left(|\nabla H|^{2}+H \Delta H+2\left\langle\nabla^{2} H, \AA \lambda+2 H^{2}|\AA|^{2}-\frac{1}{2} H^{2}|A|^{2}\right) d \sigma\right. \\
& +\int_{\Sigma}\left(L_{0} H+\frac{1}{2} H^{3}\right)\left(\left.\frac{\partial \varphi}{\partial t}\right|_{t=0}+H \varphi^{2}\right) d \sigma \\
= & 2 \int_{\Sigma} \varphi \tilde{L}_{0} \varphi d \sigma+\int_{\Sigma}\left(L_{0} H+\frac{1}{2} H^{3}\right)\left(\left.\frac{\partial \varphi}{\partial t}\right|_{t=0}+H \varphi^{2}\right) d \sigma,
\end{aligned}
$$

where the fourth-order operator $\tilde{L}_{0}$ is defined by

$$
\begin{aligned}
\tilde{L}_{0} \varphi= & \left(L_{0}\right)^{2} \varphi+\frac{1}{2} H^{2} L_{0} \varphi+2 H\left\langle\AA, \nabla^{2} \varphi\right\rangle+2 \AA(\nabla \varphi, \nabla H) \\
& +\varphi\left(|\nabla H|^{2}+H \Delta H+2\left\langle\nabla^{2} H, \AA\right\rangle+2 H^{2}|\AA|^{2}\right) .
\end{aligned}
$$


The set $\left\{P+\lambda R \mathbb{T}_{\omega}: P \in \mathbb{R}^{3}, \lambda>0, R \in S O(3), \omega \in \mathbb{D}\right\}$ made by translations, rotations and dilations of Möbius transformations of the Clifford torus $\mathbb{T}$, form an eight dimensional family of surfaces. On $\mathbb{T}_{\omega}$, we define Jacobi vector fields $Z_{0, \omega}, Z_{1, \omega}, \ldots, Z_{7, \omega}$ according to the following notation

$$
Z_{0, \omega} \text { is generated by dilations ; } \quad Z_{1, \omega}, Z_{2, \omega}, Z_{3, \omega} \text { are generated by translations ; }
$$

$$
Z_{4, \omega}, Z_{5, \omega}, Z_{6, \omega} \text { are generated by rotations ; }
$$

$Z_{7, \omega}$ is generated by the Möbius inversions described in Subsection 2.1 ,

Notice that $Z_{1, \omega}, \ldots, Z_{7, \omega}$ all induce deformations which preserve the area. We write $Z_{i, R, \omega}$ for the Jacobi vector fields on $R \mathbb{T}_{\omega}$.

Since $R \mathbb{T}_{\omega}$ is diffeomorphic to $\mathbb{T}$ by the conformal map $R T_{\omega}$, we may pull back a neighbourhood of $R \mathbb{T}_{\omega}$ in $\mathbb{R}^{3}$ onto that of $\mathbb{T}$. We write $g_{0, R, \omega}:=\left(R T_{\omega}\right)^{*} g_{0}$ for the pull back of the Euclidean metric $g_{0}$. Thus ( $\left.\mathbb{T}, g_{0, R, \omega}\right)$ is isometric to $\left(R \mathbb{T}_{\omega}, g_{0}\right)$. We also use $\bar{g}_{0, R, \omega}, n_{0, R, \omega}(p)$ and $\langle\cdot, \cdot\rangle_{L_{0, R, \omega}^{2}}$ for the tangential metric of $g_{0, R, \omega}$ on $\mathbb{T}$, the unit outer normal of $\mathbb{T}$ in $g_{0, R, \omega}$ and the $L^{2}$-inner product with metric $\bar{g}_{0, R, \omega}$. Moreover, we write $L_{0, R, \omega}^{2}(\mathbb{T})$ for $\left(L^{2}(\mathbb{T}),\langle\cdot, \cdot\rangle_{L_{0, R, \omega}^{2}}\right)$. Remark that we may regard $Z_{i, R, \omega}$ as functions on $\mathbb{T}$.

Next, we set

$$
\mathcal{K}_{0, R, \omega}:=\operatorname{span}\left\{H_{0, R, \omega}, Z_{1, R, \omega}, \ldots, Z_{7, R, \omega}\right\} \subset L_{0, R, \omega}^{2}(\mathbb{T})
$$

where $H_{0, R, \omega}$ stands for the mean curvature of $\mathbb{T}$ in $g_{0, R, \omega}$. Let us also denote by $\Pi_{0, R, \omega}: L_{0, R, \omega}^{2}(\mathbb{T}) \rightarrow$ $\left(\mathcal{K}_{0, R, \omega}\right)^{\perp \bar{g}_{0, R, \omega}}$ the $L^{2}$-projection onto $\left(\mathcal{K}_{0, R, \omega}\right)^{\perp \bar{g}_{0, R, \omega}}$ where $\perp \bar{g}_{0, R, \omega}$ stands for the orthogonality in $L_{0, R, \omega}^{2}(\mathbb{T})$ :

$$
\psi \in \mathcal{K}_{0, R, \omega}^{\perp \bar{g}_{0, R, \omega}} \Leftrightarrow\left\langle H_{0, R, \omega}, \psi\right\rangle_{L_{0, R, \omega}^{2}}=0=\left\langle Z_{i, R, \omega}, \psi\right\rangle_{L_{0, R, \omega}^{2}}(1 \leq i \leq 7) .
$$

For regular functions $\varphi: \mathbb{T} \rightarrow \mathbb{R}$, we consider the following perturbation of $\mathbb{T}$ :

$$
(\mathbb{T}[\varphi])_{R, \omega}:=\left\{p+\varphi(p) n_{0, R, \omega}(p): p \in \mathbb{T}\right\}, \quad R \mathbb{T}_{\omega}[\varphi]:=\left\{R T_{\omega}\left(p+\varphi(p) n_{0, R, \omega}(p)\right): p \in \mathbb{T}\right\}
$$

Notice that the surface $\left((\mathbb{T}[\varphi])_{R, \omega}, g_{0, R, \omega}\right)$ is isometric to $\left(R \mathbb{T}_{\omega}[\varphi], g_{0}\right)$. Concerning these perturbations, we have the following non-degeneracy property for the Willmore energy of the Clifford torus and its Möbius equivalents, see Theorem 4.2 and Corollaries 1, 2 in Weiner [43.

Proposition 2.11 (43). Let $\mathbb{T}_{\omega}$ be the immersion of $\mathbb{T}$ in $\mathbb{R}^{3}$ as in Proposition 2.5 and $\alpha \in(0,1)$ : then for all $R \in S O(3)$ and $\omega \in \mathbb{D}$, one has $W_{0}^{\prime}\left(R \mathbb{T}_{\omega}\right)=0$. Moreover, $R \mathbb{T}_{\omega}$ is non-degenerate in the following sense. For any compact set $K \subset \mathbb{D} \backslash\{0\}$ and $\ell \in \mathbb{N}$, there exist constants $C_{K, \ell, 1}>0$ and $C_{K, \ell, 2}>0$ such that for every $R \in S O(3)$ and $\omega \in K$, one has the lower bound

$$
\left\|\Pi_{0, R, \omega} \tilde{L}_{0, R, \omega} \varphi\right\|_{C^{\ell, \alpha}(\mathbb{T})} \geq C_{K, \ell, 1}\left\|\tilde{L}_{0, R, \omega} \varphi\right\|_{C^{\ell, \alpha}(\mathbb{T})} \geq C_{K, \ell, 2}\|\varphi\|_{C^{4+\ell, \alpha}(\mathbb{T})}
$$

for all $\varphi \in C^{4+\ell, \alpha}(\mathbb{T}) \cap \mathcal{K}_{0, R, \omega}^{\perp \bar{g}_{0, R, \omega}}$ where $\tilde{L}_{0, R, \omega}$ is the operator $\tilde{L}_{0}$ for $R \mathbb{T}_{\omega}$.

Proof. We first prove that

$$
\left\|\tilde{L}_{0, R, \omega} \varphi\right\|_{C^{\ell, \alpha}(\mathbb{T})} \geq C_{K, \ell}\|\varphi\|_{C^{4+\ell, \alpha}(\mathbb{T})} .
$$

We prove this by contradiction. Assume that there are $\left(R_{m}\right) \subset S O(3),\left(\omega_{m}\right) \subset K$ and $\left(\varphi_{m}\right) \subset C^{4+\ell, \alpha}(\mathbb{T})$ such that

$$
\begin{aligned}
& \left\|\varphi_{m}\right\|_{C^{4+\ell, \alpha}(\mathbb{T})}=1, \quad\left\|\tilde{L}_{0, R_{m}, \omega_{m}} \varphi_{m}\right\|_{C^{\ell, \alpha}(\mathbb{T})} \rightarrow 0, \\
& \left\langle H_{0, R_{m}, \omega_{m}}, \varphi_{m}\right\rangle_{L_{0, R_{m}, \omega_{m}}^{2}}=0=\left\langle Z_{i, R_{m} \omega_{m}}, \varphi_{m}\right\rangle_{L_{0, R_{m}, \omega_{m}}^{2}} \quad(1 \leq i \leq 7) .
\end{aligned}
$$


We may also suppose $R_{m} \rightarrow R_{0}$ and $\omega_{m} \rightarrow \omega_{0}$. Then since the embedding $C^{4+\ell, \alpha}(\mathbb{T}) \subset C^{4+\ell, \beta}(\mathbb{T})$ is compact, by (19), we also suppose that $\varphi_{m} \rightarrow \varphi_{0}$ strongly in $C^{4+\ell, \beta}(\mathbb{T})$.

Next we prove $\varphi_{0} \neq 0$. If $\varphi_{0} \equiv 0$, then from the definition of $\tilde{L}_{0, R, \omega}$, isolating the highest-order terms, we have

$$
\left(L_{0, R_{m}, \omega_{m}}\right)^{2} \varphi_{m}=f_{m}
$$

where $f_{m}$ has a strongly convergent subsequence in $C^{\ell, \alpha}(\mathbb{T})$. Thus by the elliptic regularity theory, we observe that $\left\|\varphi_{m}\right\|_{C^{4+\ell, \alpha}(\mathbb{T})} \rightarrow 0$, which contradicts (19). Hence $\varphi_{0} \not \equiv 0$.

Next, by (19) and the fact $\varphi_{m} \rightarrow \varphi_{0}$ strongly in $C^{4+\ell, \beta}(\mathbb{T})$, we notice that $\tilde{L}_{0, R_{0}, \omega_{0}} \varphi_{0}=0$ and $\varphi_{0} \in C^{\infty}(\mathbb{T})$ thanks to the elliptic regularity. By the result of Weiner [43], we have

$$
\operatorname{Ker} \tilde{L}_{0, R_{0}, \omega_{0}}=\operatorname{span}\left\{Z_{0, R_{0}, \omega_{0}}, \ldots, Z_{7, R_{0}, \omega_{0}}\right\} .
$$

We apply a Graham-Schmidt orthogonalization to $Z_{1, R_{0}, \omega_{0}}, \ldots, Z_{7, R_{0}, \omega_{0}}$ in $L_{0, R_{0}, \omega_{0}}^{2}(\mathbb{T})$ to get new variation fields $Y_{1, R_{0}, \omega_{0}}, \ldots, Y_{7, R_{0}, \omega_{0}}$. Then $\varphi_{0}$ can be expressed as

$$
\varphi_{0}=a_{0} Z_{0, R_{0}, \omega_{0}}+\sum_{i=1}^{7} a_{i} Y_{i, R_{0}, \omega_{0}} .
$$

Since $Z_{i, R_{0}, \omega_{0}}(1 \leq i \leq 7)$ are Jacobi vector fields corresponding to the area preserving deformation, it follows that

$$
\left\langle Z_{i, R_{0}, \omega_{0}}, H_{0, R_{0}, \omega_{0}}\right\rangle_{L_{0, R_{0}, \omega_{0}}^{2}}=0 \quad(1 \leq i \leq 7) .
$$

By (19) and the definitions of $\left\{Y_{i, R_{0}, \omega_{0}}\right\}_{1 \leq i \leq 7}$, one observes that

$$
0=\left\langle\varphi_{0}, H_{0, R_{0}, \omega_{0}}\right\rangle_{L_{0, R_{0}, \omega_{0}}^{2}}=a_{0}\left\langle Z_{0, R_{0}, \omega_{0}}, H_{0, R_{0}, \omega_{0}}\right\rangle_{L_{0, R_{0}, \omega_{0}}^{2}} .
$$

Noting that $Z_{0, R_{0}, \omega_{0}}$ is generated by dilations, we obtain

$$
0<8 \sqrt{2} \pi^{2}=\left.\frac{d}{d t}(1+t)^{2}\left|R_{0} \mathbb{T}_{\omega_{0}}\right|_{g_{0}}\right|_{t=0}=\left.\frac{d}{d t}\left|(1+t)^{2} R_{0} \mathbb{T}_{\omega_{0}}\right|_{g_{0}}\right|_{t=0}=\left\langle Z_{0, R_{0}, \omega_{0}}, H_{0, R_{0}, \omega_{0}}\right\rangle_{L_{0, R_{0}, \omega_{0}}^{2}} .
$$

Hence, from (20), we deduce that $a_{0}=0$ and $\varphi_{0}=\sum_{i=1}^{7} a_{i} Y_{i, R_{0}, \omega_{0}}$. Due to (19) and the definitions of $\left\{Y_{i, R_{0}, \omega_{0}}\right\}_{1 \leq i \leq 7}$, we have $\left\langle\varphi_{0}, Y_{i, R_{0}, \omega_{0}}\right\rangle_{L_{0, R_{0}, \omega_{0}}^{2}}=0(1 \leq i \leq 7)$, which yields $a_{i}=0(1 \leq i \leq 7)$. However, this contradicts $\varphi_{0} \not \equiv 0$. Thus (18) holds.

Next we show that

$$
\left\|\Pi_{0, R, \omega} \tilde{L}_{0, R, \omega} \varphi\right\|_{C^{\ell, \alpha}(\mathbb{T})} \geq C_{K, \ell}\left\|\tilde{L}_{0, R, \omega} \varphi\right\|_{C^{\ell, \alpha}(\mathbb{T})} .
$$

Again we argue by contradiction and suppose that there are $\left(R_{m}\right) \subset S O(3),\left(\omega_{m}\right) \subset K$ and $\left(\varphi_{m}\right) \subset$ $C^{4+\ell, \alpha}(\mathbb{T})$ such that

$$
\begin{aligned}
& \left\|\Pi_{0, R_{m}, \omega_{m}} \tilde{L}_{0, R_{m}, \omega_{m}} \varphi_{m}\right\|_{C^{\ell, \alpha}(\mathbb{T})} \rightarrow 0, \quad\left\|\tilde{L}_{0, R_{m}, \omega_{m}} \varphi_{m}\right\|_{C^{\ell, \alpha}(\mathbb{T})}=1, \\
& \left\langle H_{0, R_{m}, \omega_{m}}, \varphi_{m}\right\rangle_{L_{0, R_{m}, \omega_{m}}^{2}}=0=\left\langle Z_{i, R_{m} \omega_{m}}, \varphi_{m}\right\rangle_{L_{0, R_{m}, \omega_{m}}^{2}} \quad(1 \leq i \leq 7) .
\end{aligned}
$$

By (18), we observe that $\left(\left\|\varphi_{m}\right\|_{C^{4+\ell, \alpha}(\mathbb{T})}\right)$ is bounded. As above, we use the $L_{0, R_{m}, \omega_{m}}^{2}(\mathbb{T})$-orthogonal system $\left\{Y_{i, R_{m}, \omega_{m}}\right\}_{1 \leq i \leq 7}$ starting from $\left\{Z_{i, R_{m}, \omega_{m}}\right\}_{1 \leq i \leq 7}$. Since $\left\langle Y_{i, R_{m}, \omega_{m}}, H_{0, R_{m}, \omega_{m}}\right\rangle_{L_{0, R_{m}, \omega_{m}}^{2}}=0$ holds due to the area preserving properties, set $Y_{0, R_{m}, \omega_{m}}:=H_{0, R_{m}, \omega_{m}} /\left\|H_{0, R_{m}, \omega_{m}}\right\|_{L_{0, R_{m}, \omega_{m}}^{2}}$. Then $\left(Y_{i, R_{m}, \omega_{m}}\right)_{0 \leq i \leq 7}$ is an $L_{0, R_{m}, \omega_{m}}^{2}(\mathbb{T})$-orthogonal system. Using $\left(Y_{i, R_{m}, \omega_{m}}\right)_{0 \leq i \leq 7}, \Pi_{0, R_{m}, \omega_{m}} \tilde{L}_{0, R_{m}, \omega_{m}} \varphi_{m}$ is written as

$$
\Pi_{0, R_{m}, \omega_{m}} \tilde{L}_{0, R_{m}, \omega_{m}} \varphi_{m}=\tilde{L}_{0, R_{m}, \omega_{m}} \varphi_{m}-\sum_{i=0}^{7}\left\langle\tilde{L}_{0, R_{m}, \omega_{m}} \varphi_{m}, Y_{i, R_{m}, \omega_{m}}\right\rangle_{L_{0, R_{m}, \omega_{m}}^{2}} Y_{i, R_{m}, \omega_{m}} .
$$


Since $Y_{i, R_{m}, \omega_{m}} \in \operatorname{Ker} \tilde{L}_{0, R_{m}, \omega_{m}}(1 \leq i \leq 7)$, we get

$$
\left\langle\tilde{L}_{0, R_{m}, \omega_{m}} \varphi_{m}, Y_{i, R_{m}, \omega_{m}}\right\rangle_{L_{0, R_{m}, \omega_{m}}^{2}}=\left\langle\varphi_{m}, \tilde{L}_{0, R_{m}, \omega_{m}} Y_{i, R_{m}, \omega_{m}}\right\rangle_{L_{0, R_{m}, \omega_{m}}^{2}}=0
$$

for all $1 \leq i \leq 7$. Thus

$$
\Pi_{0, R_{m}, \omega_{m}} \tilde{L}_{0, R_{m}, \omega_{m}} \varphi_{m}=\tilde{L}_{0, R_{m}, \omega_{m}} \varphi_{m}-\left\langle\tilde{L}_{0, R_{m}, \omega_{m}} \varphi_{m}, Y_{0, R_{m}, \omega_{m}}\right\rangle_{L_{0, R_{m}, \omega_{m}}^{2}} Y_{0, R_{m}, \omega_{m}} .
$$

Noting that $\left\langle\tilde{L}_{0, R_{m}, \omega_{m}} \varphi_{m}, Z_{0, R_{m}, \omega_{m}}\right\rangle_{L_{0, R_{m}, \omega_{m}}^{2}}=\left\langle\varphi_{m}, \tilde{L}_{0, R_{m}, \omega_{m}} Z_{0, R_{m}, \omega_{m}}\right\rangle_{L_{0, R_{m}, \omega_{m}}^{2}}=0$ and taking an $L_{0, R_{m}, \omega_{m}}^{2}(\mathbb{T})$-inner product of $\Pi_{0, R_{m}, \omega_{m}} \tilde{L}_{0, R_{m}, \omega_{m}} \varphi_{m}$ and $Z_{0, R_{m}, \omega_{m}}$, we observe that

$$
\begin{aligned}
& \left\langle\Pi_{0, R_{m}, \omega_{m}} \tilde{L}_{0, R_{m}, \omega_{m}} \varphi_{m}, Z_{0, R_{m}, \omega_{m}}\right\rangle_{L_{0, R_{m}, \omega_{m}}^{2}} \\
= & -\left\langle\tilde{L}_{0, R_{m}, \omega_{m}} \varphi_{m}, Y_{0, R_{m}, \omega_{m}}\right\rangle_{L_{0, R_{m}, \omega_{m}}^{2}}\left\langle Y_{0, R_{m}, \omega_{m}}, Z_{0, R_{m}, \omega_{m}}\right\rangle_{L_{0, R_{m}, \omega_{m}}^{2}} .
\end{aligned}
$$

Recalling (21) and (23), we infer that

$$
\left\langle\tilde{L}_{0, R_{m}, \omega_{m}} \varphi_{m}, Y_{0, R_{m}, \omega_{m}}\right\rangle_{L_{0, R_{m}, \omega_{m}}^{2}} \rightarrow 0 .
$$

Thus from (24) we get a contradiction:

$$
1=\left\|\tilde{L}_{0, R_{m}, \omega_{m}} \varphi_{m}\right\|_{C^{\ell, \alpha}(\mathbb{T})} \rightarrow 0 .
$$

Hence, (22) holds and we complete the proof.

Remark 2.12. When $\omega=0, \mathbb{T}_{\omega}$ is the symmetric Clifford torus, so the action of one among the rotation vector fields is trivial. A global version of Proposition 2.11, including also the case $\omega=0$, can be expressed as the non-degeneracy of the family of inverted (and rotated) Clifford tori in the sense of Bott. In particular the constants $C_{K, 1}$ and $C_{K, 2}$ in (17) remains controlled when $\omega$ approaches zero. For instance, when $R=\mathrm{Id}$ and $Z_{0, R, 6}$ corresponds to the rotation along the $z$-axis, writing $\omega=(x, y)$, it suffices to replace the definition of $\mathcal{K}_{0, \mathrm{Id}, \omega}$ by

$$
\mathcal{K}_{0, \mathrm{Id}, \omega}:=\operatorname{span}\left\{H_{0, \mathrm{Id}, \omega}, Z_{1, \mathrm{Id}, \omega}, \ldots, Z_{5, \mathrm{Id}, \omega}, \frac{\partial}{\partial x} T_{\omega}, \frac{\partial}{\partial y} T_{\omega}\right\}
$$

near $\omega=0$.

\section{Finite-dimensional reduction}

In this section we reduce the problem to a finite-dimensional one, namely to the choice of the concentration point and to the Möbius action on the scaled Clifford tori. We first introduce a family of approximate solutions to our problem, and then we modify them properly to solve the equation up to elements in the Kernel of the operator $\tilde{L}$. In the next section we will adjust the parameters so that the Willmore equation is fully solved (under area constraint).

\subsection{Approximate solutions: small tori embedded in $M$}

We fix a compact set $K$ of the unit disk $\mathbb{D}$ and we consider then the family

$$
\hat{\mathcal{T}}_{\varepsilon, K}=\left\{\varepsilon R \mathbb{T}_{\omega}: R \in S O(3), \omega \in K\right\} .
$$

We remark that, by construction, elements in $\hat{\mathcal{T}}_{\varepsilon, K}$ consist of Willmore surfaces in $\mathbb{R}^{3}$ all with area identically equal to $4 \sqrt{2} \pi^{2} \varepsilon^{2}$. 
Remark 3.1. Notice that, by rotation invariance of the Clifford torus $\mathbb{T}$, the above family $\hat{\mathcal{T}}_{\varepsilon, K}$ is fourdimensional and not five-dimensional.

Next, we construct a family of surfaces in $M$ by exponential maps of $\hat{\mathcal{T}}_{\varepsilon, K}$. Fix $P \in M$. Around $P$, we may find a local orthonormal frame $\left\{F_{P, 1}, F_{P, 2}, F_{P, 3}\right\}$. By this frame, we may identify $T_{P} M$ with $\mathbb{R}^{3}$ and define the exponential map $\exp _{P}^{g}$. Since $M$ is compact, there exists a $\rho_{0}>0$, which is independent of $P \in M$, such that $\exp _{P}$ is diffeomorphic on $B_{\rho_{0}}(0) \subset \mathbb{R}^{3}$ to $\exp _{P}\left(B_{\rho_{0}}(0)\right)$ for every $P \in M$. Then we may select $\varepsilon_{0}>0$ so that

$$
\varepsilon R \mathbb{T}_{\omega} \subset B_{\rho_{0}}(0) \quad \text { for every }(\varepsilon, R, \omega) \in\left(0, \varepsilon_{0}\right] \times S O(3) \times \mathbb{D} .
$$

Hereafter, we fix $\varepsilon_{0}>0$ and consider the case $\varepsilon \in\left(0, \varepsilon_{0}\right)$. For a compact set $K \subset \mathbb{D}$, we define

$$
\mathcal{T}_{\varepsilon, K}=\left\{\exp _{P}(\Sigma): P \in M, \Sigma \in \hat{\mathcal{T}}_{\varepsilon, K}\right\} \quad \text { for } \varepsilon \in\left(0, \varepsilon_{0}\right) .
$$

Before proceeding further, we make some remarks. Since we are interested in the asymptotic behaviour of the family satisfying the small area constraint, it is useful to introduce the following metric on $M$ :

$$
g_{\varepsilon}(P):=\frac{1}{\varepsilon^{2}} g(P) .
$$

As above, by (25), putting $F_{\varepsilon, P, \alpha}:=\varepsilon F_{P, \alpha},\left\{F_{\varepsilon, P, 1}, F_{\varepsilon, P, 2}, F_{\varepsilon, P, 3}\right\}$ is a local orthonormal frame with metric $g_{\varepsilon}$ and using this frame, we may define the exponential map $\exp _{P}^{g_{\varepsilon}}$. Let us denote by $g_{P}$ and $g_{\varepsilon, P}$ the pull back of the metrics $g$ and $g_{\varepsilon}$ through $\exp _{P}^{g}$ and $\exp _{P}^{g_{\varepsilon}}: g_{P}:=\left(\exp _{P}^{g}\right)^{*} g$ and $g_{\varepsilon, P}:=\left(\exp _{P}^{g_{\varepsilon}}\right)^{*} g_{\varepsilon}$. Then it is easily seen that

(i) Write $W_{g}$ and $W_{g_{\varepsilon}}$ for the Willmore functional on $(M, g)$ and $\left(M, g_{\varepsilon}\right)$. Let $\Sigma \subset M$ be an embedded surface. Denote by $H_{g}$ and $H_{g_{\varepsilon}}$ the mean curvature of $\Sigma$ with the metric $g$ and $g_{\varepsilon}$, respectively. Then we have

$$
H_{g_{\varepsilon}}=\varepsilon H_{g}, \quad W_{g_{\varepsilon}}(\Sigma)=W_{g}(\Sigma), \quad W_{g_{\varepsilon}}^{\prime}(\Sigma)=\varepsilon^{3} W_{g}^{\prime}(\Sigma) .
$$

In particular, $\Sigma$ is a Willmore surface with the area constraint in $(M, g)$ if and only if so is in $\left(M, g_{\varepsilon}\right)$.

(ii) The exponential map $\exp _{P}^{g_{\varepsilon}}$ is defined in $B_{\varepsilon^{-1}} \rho_{0}(0)$ and

$$
\exp _{P}^{g}(\varepsilon z)=\exp _{P}^{g_{\varepsilon}}(z) \quad \text { for all }|z|_{g_{0}} \leq \varepsilon^{-1} \rho_{0} .
$$

(iii) The metric $g_{P}$ has the following expansion (see, for instance, Lee-Parker [22]):

$$
g_{P, \alpha \beta}(x)=\delta_{\alpha \beta}+\frac{1}{3} R_{\alpha \mu \nu \beta} x^{\mu} x^{\nu}+R(x)=: \delta_{\alpha \beta}+\tilde{h}_{P, \alpha \beta}(x) \quad \text { in } \overline{B_{\rho_{0}}(0)} \subset T_{P} M
$$

where $R(x)$ is the remainder term satisfying $\left|\nabla^{k} R(x)\right|=O\left(|x|^{3-k}\right)$ for $k=0,1,2,3$. Moreover, since $M$ is three dimensional, one sees that

$$
\begin{aligned}
R_{\alpha \mu \nu \beta} & =g_{P}\left(\operatorname{Riem}\left(\partial_{\nu}, \partial_{\beta}\right) \partial_{\mu}, \partial_{\alpha}\right) \\
& =\frac{\operatorname{Sc}_{P}}{2}\left(g_{P, \alpha \beta} g_{P, \mu \nu}-g_{P, \alpha \nu} g_{P, \mu \beta}\right)+g_{P, \alpha \nu} R_{\mu \beta}-g_{P, \alpha \beta} R_{\mu \nu}+g_{P, \mu \beta} R_{\alpha \nu}-g_{P, \mu \nu} R_{\alpha \beta}
\end{aligned}
$$

where $\operatorname{Sc}_{P}$ and $R_{\alpha \beta}$ are the scalar curvature and the Ricci tensor evaluated at $P$, respectively.

(iv) For $g_{\varepsilon, P}$, we have

$$
g_{\varepsilon, P, \alpha \beta}(y)=\delta_{\alpha \beta}+\varepsilon^{2} h_{P, \alpha \beta}^{\varepsilon}(y) \quad \text { for any }|y|_{g_{0}} \leq \varepsilon^{-1} \rho_{0}
$$


where $h_{P, \alpha \beta}^{\varepsilon}(y)=\varepsilon^{-2} \tilde{h}_{P, \alpha \beta}(\varepsilon y)$ and there holds

$$
|y|^{-2}\left|h_{P, \alpha \beta}^{\varepsilon}\right|+|y|^{-1}\left|\nabla h_{P, \alpha \beta}^{\varepsilon}\right|+\sum_{i=2}^{k}\left|\nabla^{i} h_{P, \alpha \beta}^{\varepsilon}(y)\right|_{g_{0}} \leq \tilde{h}_{0, k}
$$

for any $k \in \mathbb{N}$ and $|y|_{g_{0}} \leq \varepsilon^{-1} \rho_{0}$. Here $\tilde{h}_{0, k}$ depends on $k$, but not on $\varepsilon$. Moreover, $(P, y) \mapsto$ $g_{\varepsilon, P, \alpha \beta}(y)$ is smooth and for any $k, \ell \in \mathbb{N}$, there exists a $C_{k, \ell}>0$ such that

$$
|y|^{-2}\left|D_{P}^{k+1} g_{\varepsilon, P, \alpha \beta}(y)\right|+|y|^{-1}\left|D_{P}^{k+1} \nabla_{y} g_{\varepsilon, P, \alpha \beta}(y)\right|+\sum_{j=2}^{\ell}\left|D_{P}^{k+1} \nabla_{y}^{j} g_{\varepsilon, P, \alpha \beta}(y)\right| \leq C_{k, \ell} \varepsilon^{2}
$$

where $D_{P}$ denotes the differential by $P$ in the original scale of $M$ (not in the rescaled one).

(v) The family $\mathcal{T}_{\varepsilon, K}$ is rewritten as

$$
\begin{aligned}
\mathcal{T}_{\varepsilon, K} & =\left\{\exp _{P}^{g}\left(\varepsilon R \mathbb{T}_{\omega}\right): P \in M, R \in S O(3), \omega \in K\right\} \\
& =\left\{\exp _{P}^{g_{\varepsilon}}\left(R \mathbb{T}_{\omega}\right): P \in M, R \in S O(3), \omega \in K\right\} .
\end{aligned}
$$

Remark 3.2. Möbius-inverted Clifford tori in $\mathbb{R}^{3}$ can be clearly completely described by their translation vector, their rotation and their distortion factor. It then follows that for exponential maps in the metric $g_{\varepsilon}$ (see (25)), except for $\omega=0$, the parameters $P, R, \omega$ give a local smooth parametrization of $\mathcal{T}_{\varepsilon, K}$.

As for Remark 2.12. $\mathcal{T}_{\varepsilon, K}$ turns out to be globally a smooth manifold even near surfaces described by $\omega=0$, where one could use a different local parametrization.

In what follows, we always consider our problem on $\left(M, g_{\varepsilon}\right)$. For example, let $\mathcal{S}_{\varepsilon} \subset M$ be a family of smooth surfaces with $|\Sigma|_{g}=C_{0} \varepsilon^{2}$ for each $\Sigma \in \mathcal{S}_{\varepsilon}$. Using $g_{\varepsilon}$, we can work on the rescaled surfaces with the fixed area $C_{0}$ in normal coordinate of $g_{\varepsilon}$.

Next we shall show that each element of $\mathcal{T}_{\varepsilon, K}$ is an approximate solution of $W_{g_{\varepsilon}}$.

Lemma 3.3. Fix a compact set $K \subset \mathbb{D}$ and $k \in \mathbb{N}$. Then there exists $C_{K, k}>0$ such that

$$
\left\|W_{g_{\varepsilon}}^{\prime}(\Sigma)\right\|_{C^{k}(\Sigma)} \leq C_{K, k} \varepsilon^{2} \quad \text { for every } \Sigma \in \mathcal{T}_{\varepsilon, K} \quad \text { and } \quad \varepsilon \in\left(0, \varepsilon_{0}\right) .
$$

Proof. By Proposition 2.8, we have

$$
W_{g_{\varepsilon}}^{\prime}(\Sigma)=-\Delta_{g_{\varepsilon}} H_{g_{\varepsilon}}-\left|A_{g_{\varepsilon}}\right|^{2} H_{g_{\varepsilon}}-H_{g_{\varepsilon}} \operatorname{Ric}_{g_{\varepsilon}}\left(n_{g_{\varepsilon}}, n_{g_{\varepsilon}}\right)+\frac{1}{2} H_{g_{\varepsilon}}^{3}
$$

in the sense that $\delta W_{g_{\varepsilon}}(\Sigma)[\varphi]=\int_{\Sigma} W_{g_{\varepsilon}}^{\prime}(\Sigma) \varphi d \sigma_{g_{\varepsilon}}$. To prove the lemma we apply this formula to a surface $\Sigma \in \tilde{\mathcal{T}}_{\varepsilon, K}$ endowed with the metric induced by $g_{\varepsilon}$ defined in (25) (or (28)).

First, by the properties of $\rho_{0}$ and $\varepsilon_{0}$, and the definition of $g_{\varepsilon}$, we may find $\rho_{K}>0$ such that

$$
|x|_{g_{0}} \leq \rho_{K} \leq \frac{\rho_{0}}{\varepsilon} \quad \text { for each } x \in R \mathbb{T}_{\omega} \subset T_{P} M \quad \text { and } \quad(\varepsilon, P, R, \omega) \in\left(0, \varepsilon_{0}\right] \times M \times S O(3) \times K .
$$

Moreover, from (28) and (29), for each $k \in \mathbb{N}$, there exists $C_{K, k}>0$ such that

$$
\left\|g_{\varepsilon, P, \alpha \beta}-\delta_{\alpha \beta}\right\|_{C^{k}\left(\overline{B_{\rho_{K}}(0)}\right)} \leq C_{K, k} \varepsilon^{2} \quad \text { for each }(\varepsilon, P, R, \omega) \in\left(0, \varepsilon_{0}\right] \times M \times S O(3) \times K .
$$

By (33), we may compare the geometric quantities with the Euclidean ones and obtain the following estimates:

$$
\begin{aligned}
& \left\|\Gamma_{\varepsilon, P, \mu \nu}^{\xi}\right\|_{C^{k}\left(\overline{B_{\rho_{K}}(0)}\right)} \leq C_{K, k} \varepsilon^{2}, \quad\left\|\operatorname{Ric}_{\varepsilon, P}\right\|_{C^{k}\left(\overline{B_{\rho_{K}}(0)}\right)} \leq C_{K, k} \varepsilon^{2}, \\
& \left\|A_{\varepsilon, P, R, \omega}-A_{0, R, \omega}\right\|_{C^{k}\left(R \mathbb{T}_{\omega}\right)} \leq C_{K, k} \varepsilon^{2}, \quad\left\|\left(\Delta_{\varepsilon, P, R, \omega}-\Delta_{0, R, \omega}\right) \varphi\right\|_{C^{k}\left(R \mathbb{T}_{\omega}\right)} \leq C_{K, k} \varepsilon^{2}\|\varphi\|_{C^{k+2}\left(R \mathbb{T}_{\omega}\right)}
\end{aligned}
$$


for each $(\varepsilon, P, R, \omega) \in\left(0, \varepsilon_{0}\right] \times M \times S O(3) \times K$ and $\varphi \in C^{k+2}\left(R \mathbb{T}_{\omega}\right)$. Here $\Gamma_{\varepsilon, P, \mu \nu}^{\xi}, \operatorname{Ric}_{\varepsilon, P}, A_{\varepsilon, P, R, \omega}$, $A_{0, R, \omega}, \Delta_{\varepsilon, P, R, \omega}$ and $\Delta_{0, R, \omega}$ stand for the Christoffel symbol and the Ricci tensor of $\left(B_{\rho_{K}}(0), g_{\varepsilon, P}\right)$, and the second fundamental form and the Laplace-Beltrami operator of $R \mathbb{T}_{\omega}$ with $g_{\varepsilon, P}$ and $g_{0}$, respectively.

Now, from (32), (33) and (34), it follows that

$$
\left\|W_{g_{\varepsilon}}^{\prime}(\Sigma)-W_{g_{0}}^{\prime}\left(R \mathbb{T}_{\omega}\right)\right\|_{C^{k}\left(R \mathbb{T}_{\omega}\right)} \leq C_{K, k} \varepsilon^{2} .
$$

Recalling that the Clifford torus (and all its images under Möbius transformation, by conformal invariance of $W$ in $\left.\mathbb{R}^{3}\right)$ is a critical point of the Willmore functional, namely $W_{g_{0}}^{\prime}\left(R \mathbb{T}_{\omega}\right)=0$, Lemma 3.3 follows.

\subsection{Reduction to a finite-dimensional problem}

Let $R \in S O(3)$ and $\omega \in \mathbb{D}$. As in Subsection 2.2 we pull back a neighbourhood of $R \mathbb{T}_{\omega}$ with the metric $g_{\varepsilon, P}$ onto that of $\mathbb{T}$. Namely, we define $g_{\varepsilon, P, R, \omega}$ by the pull back of $g_{\varepsilon, P}$ via the map $R \circ T_{\omega}$ : $g_{\varepsilon, P, R, \omega}:=\left(R \circ T_{\omega}\right)^{*} g_{\varepsilon, P}=T_{\omega}^{*} \circ R^{*} \circ\left(\exp _{P}^{g_{\varepsilon}}\right)^{*} g_{\varepsilon}$. Remark that $\left(\mathbb{T}, g_{\varepsilon, P, R, \omega}\right)$ is isometric to $\left(\exp _{P}^{g_{\varepsilon}}\left(R \mathbb{T}_{\omega}\right), g_{\varepsilon}\right)$ or $\left(R \mathbb{T}_{\omega}, g_{\varepsilon, P}\right)$. Let $n_{\varepsilon, P, R, \omega}$ be the unit outer normal of $\left(\mathbb{T}, g_{\varepsilon, P, R, \omega}\right)$.

As before, we consider perturbations of $\left(\mathbb{T}, g_{\varepsilon, P, R, \omega}\right)$ by regular functions $\varphi: \mathbb{T} \rightarrow \mathbb{R}$ :

$$
\begin{aligned}
(\mathbb{T}[\varphi])_{\varepsilon, P, R, \omega} & :=\left\{p+\varphi(p) n_{\varepsilon, P, R, \omega}(p): p \in \mathbb{T}\right\}, \\
\left(R \mathbb{T}_{\omega}[\varphi]\right)_{\varepsilon, P} & :=\left\{R T_{\omega}\left(p+\varphi(p) n_{\varepsilon, P, R, \omega}(p)\right): p \in \mathbb{T}\right\}, \quad \Sigma_{\varepsilon, P, R, \omega}[\varphi]:=\exp _{P}^{g_{\varepsilon}}\left(\left(R \mathbb{T}_{\omega}[\varphi]\right)_{\varepsilon, P}\right) .
\end{aligned}
$$

Given a positive constant $\bar{C}$, we next define the family of functions

$$
\mathcal{M}_{\varepsilon, P, R, \omega}=\left\{\varphi \in C^{4, \alpha}(\mathbb{T}):\|\varphi\|_{C^{4, \alpha}(\mathbb{T})} \leq \bar{C} \varepsilon^{2} \text { and such that }\left|\Sigma_{\varepsilon, P, R, \omega}[\varphi]\right|_{g_{\varepsilon}}=4 \sqrt{2} \pi^{2}\right\} .
$$

Since each element of $\mathcal{T}_{\varepsilon, K}$ is described as $\Sigma_{\varepsilon, P, R, \omega}[0]$, we notice that, in metric $g_{\varepsilon}$, the Hausdorff distance of elements $\Sigma_{\varepsilon, P, R, \omega}[\varphi]\left(\varphi \in \mathcal{M}_{\varepsilon, P, R, \omega}\right.$ and $\left.\omega \in K\right)$ from the previous approximate solutions $\mathcal{T}_{\varepsilon, K}$ is of order $\varepsilon^{2}$, with a constant depending on $\bar{C}$ and $K$.

We next define $\mathcal{M}_{\varepsilon}$ to be the Banach manifold of surfaces of the type $\Sigma_{\varepsilon, P, R, \omega}[\varphi]$ with $P$ varying in $M$, $\varphi \in \mathcal{M}_{\varepsilon, P, R, \omega}, R$ in $S O(3)$ and $\omega$ in $\mathbb{D}$. Notice that, by construction, elements in $\mathcal{M}_{\varepsilon}$ all satisfy the desired area constraint.

The surfaces in $\mathcal{T}_{\varepsilon, K}$ form a seven-dimensional sub-manifold in $\mathcal{M}_{\varepsilon}$ : we will show that the Willmore functional is indeed non-degenerate in directions orthogonal to $\mathcal{T}_{\varepsilon, K}$.

To observe the non-degeneracy of elements in $\mathcal{T}_{\varepsilon, K}$, we introduce the eight-dimensional vector space

$$
\mathcal{K}_{\varepsilon, P, R, \omega}^{\varphi}:=\operatorname{span}\left\{H_{\varepsilon, P, R, \omega}[\varphi], Z_{1, R, \omega}, \ldots, Z_{7, R, \omega}\right\}
$$

where $\varphi \in C^{4, \alpha}(\mathbb{T})$ and $H_{\varepsilon, P, R, \omega}[\varphi]$ denotes the mean curvature of $\Sigma_{\varepsilon, P, R, \omega}[\varphi]$ in the metric $g_{\varepsilon}$. Since we are only interested in $\varphi$ whose $C^{4, \alpha}$-norm is small, $\Sigma_{\varepsilon, P, R, \omega}[\varphi]$ can be written as the normal graph on $\mathbb{T}$ and this correspondence is diffeomorphic. Therefore, we pull back geometric quantities of $\Sigma_{\varepsilon, P, R, \omega}[\varphi]$ onto $\mathbb{T}$. In particular, we write $\bar{g}_{\varepsilon, P, R, \omega}[\varphi]$ for pull back of the tangential metric of $\Sigma_{\varepsilon, P, R, \omega}[\varphi]$ on $\mathbb{T}$. Denote by $\langle\cdot, \cdot\rangle_{\varepsilon, P, R, \omega, \varphi}$ and $L_{\varepsilon, P, R, \omega, \varphi}^{2}(\mathbb{T})$ the $L^{2}$-inner product of $L^{2}(\mathbb{T})$ with the metric $\bar{g}_{\varepsilon, P, R, \omega}[\varphi]$ and $\left(L^{2}(\mathbb{T}),\langle\cdot, \cdot\rangle_{\varepsilon, P, R, \omega, \varphi}\right)$. Remark that when $\varepsilon=0$ and $\varphi=0$, these symbols coincide with those introduced in Subsection 2.2 and do not depend on $P \in M$, i.e., $g_{0, P, R, \omega}=g_{0, R, \omega}$ and so on. See the comments above Proposition 2.11]

Next, as in the proof of Proposition 2.11, we normalize and orthogonalize $H_{\varepsilon, P, R, \omega}[\varphi]$ and $\left\{Z_{i, R, \omega}\right\}_{i=1}^{7}$ in $L_{\varepsilon, P, R, \omega, \varphi}^{2}(\mathbb{T})$. Namely, we first normalize and orthogonalize $\left\{Z_{i, R, \omega}\right\}_{i=1}^{7}$ to get $\left\{Y_{i, \varepsilon, P, R, \omega}[\varphi]\right\}_{i=1}^{7}$. Then we obtain $Y_{0, \varepsilon, P, R, \omega}[\varphi]$ from $H_{\varepsilon, P, R, \omega}[\varphi]$ and $\left\{Y_{i, \varepsilon, P, R, \omega}[\varphi]\right\}_{i=1}^{7}$, and we may assume that

$$
\left\{Y_{0, \varepsilon, P, R, \omega}[\varphi], Y_{1, \varepsilon, P, R, \omega}[\varphi], \ldots, Y_{7, \varepsilon, P, R, \omega}[\varphi]\right\}
$$


is the $L_{\varepsilon, P, R, \omega, \varphi}^{2}(\mathbb{T})$-orthonormal basis of $\mathcal{K}_{\varepsilon, P, R, \omega}^{\varphi}$. We also define the $L_{\varepsilon, P, R, \omega, \varphi}^{2}(\mathbb{T})$-projection to the $\operatorname{space}\left(\mathcal{K}_{\varepsilon, P, R, \omega}^{\varphi}\right)^{\perp \bar{g}_{\varepsilon, P, R, \omega}[\varphi]}$ by

$$
\begin{aligned}
& \Pi_{\varepsilon, P, R, \omega}^{\varphi}: L_{\varepsilon, P, R, \omega, \varphi}^{2}(\mathbb{T}) \rightarrow\left(\mathcal{K}_{\varepsilon, P, R, \omega}^{\varphi}\right)^{\perp \bar{g}_{\varepsilon, P, R, \omega}[\varphi]}, \\
& \Pi_{\varepsilon, P, R, \omega}^{\varphi} \psi:=\psi-\sum_{i=0}^{7}\left\langle\psi, Y_{i, \varepsilon, P, R, \omega}[\varphi]\right\rangle_{L_{\varepsilon, P, R, \omega, \varphi}^{2}} Y_{i, \varepsilon, P, R, \omega}[\varphi] .
\end{aligned}
$$

Finally, we also define $Y_{i, R, \omega}[\varphi]$ and $\Pi_{0, R, \omega}^{\varphi}$ for the Euclidean metric in a similar way to above. Remark that $Y_{i, 0, P, R, \omega}[\varphi]=Y_{i, R, \omega}[\varphi], \Pi_{0, P, R, \omega}^{\varphi}=\Pi_{0, R, \omega}^{\varphi}$ and $\Pi_{0, R, \omega}^{0}=\Pi_{0, R, \omega}$ hold.

Regarding the properties of $\left\{Y_{i, \varepsilon, P, R, \omega}[\varphi]\right\}_{i=0}^{7}$ and $\Pi_{\varepsilon, P, R, \omega}^{\varphi}$, we have

Lemma 3.4. Fix a compact set $K \subset \mathbb{D}, \ell \in \mathbb{N}$ and $\alpha \in(0,1)$. Then there exist $r_{K, \ell, 1}>0, \varepsilon_{K, \ell, 1}>0$ and $C_{K, \ell}$ such that

(a) For each $i=0, \ldots, 7$ and $\varepsilon \in\left(0, \varepsilon_{K, \ell, 1}\right]$, the map

$$
\overline{B_{r_{K, \ell, 1}, C^{4+\ell, \alpha}}(0)} \ni \varphi \mapsto Y_{i, \varepsilon, P, R, \omega}[\varphi] \in C^{2+\ell, \alpha}(\mathbb{T})
$$

is smooth and $D_{\varphi}^{\ell}\left(Y_{i, \varepsilon, P, R, \omega}[\varphi]\right)$ is a bounded map in $\overline{B_{r_{K, 1}, C^{4+\ell, \alpha}}(0)}$ and the bounds only depend on $r_{K, \ell, 1}$, $K$ and $\varepsilon_{K, \ell, 1}$. Here $B_{r_{K, \ell, 1}, C^{k, \alpha}}(0)$ denotes the ball in $C^{k, \alpha}(\mathbb{T})$ centred at the origin with the radius $r_{K, \ell, 1}$ and $D_{\varphi}$ does the derivative with respect to $\varphi$. In particular, the map

$$
\overline{B_{r_{K, 1}, C^{4+\ell, \alpha}}(0)} \ni \varphi \mapsto \Pi_{\varepsilon, P, R, \omega}^{\varphi} \in \mathcal{L}\left(C^{2+\ell, \alpha}(\mathbb{T}), C^{2+\ell, \alpha}(\mathbb{T})\right)
$$

is smooth and $D_{\varphi}^{\ell} \Pi_{\varepsilon, P, R, \omega}^{\varphi}$ is bounded and the bounds also depend only on $r_{K, 1}, K$ and $\varepsilon_{K, 1}$.

(b) For every $(\varepsilon, P, R, \omega) \in\left(0, \varepsilon_{K, 1}\right] \times M \times S O(3) \times K$ and $\|\varphi\|_{C^{4+\ell, \alpha}(\mathbb{T})} \leq r_{K, 1}$, there holds

$$
\begin{gathered}
\left\|Y_{i, \varepsilon, P, R, \omega}[\varphi]-Y_{i, R, \omega}[\varphi]\right\|_{C^{2+\ell, \alpha}(\mathbb{T})}+\left\|D_{\varphi}\left(Y_{i, \varepsilon, P, R, \omega}[\varphi]\right)-D_{\varphi}\left(Y_{i, R, \omega}[\varphi]\right)\right\|_{\mathcal{L}\left(C^{4+\ell, \alpha}(\mathbb{T}), C^{2+\ell, \alpha}(\mathbb{T})\right)} \\
+\left\|D_{\varphi}^{2}\left(Y_{i, \varepsilon, P, R, \omega}[\varphi]\right)-D_{\varphi}^{2}\left(Y_{i, R, \omega}[\varphi]\right)\right\|_{\mathcal{L}^{2}\left(C^{4+\ell, \alpha}(\mathbb{T}), C^{2+\ell, \alpha}(\mathbb{T})\right)} \leq C_{K, \ell} \varepsilon^{2}
\end{gathered}
$$

where $\mathcal{L}^{2}\left(C^{4+\ell, \alpha}(\mathbb{T}), C^{2+\ell, \alpha}(\mathbb{T})\right)$ is a Banach space of all multilinear maps from $C^{4+\ell, \alpha}(\mathbb{T}) \times C^{4+\ell, \alpha}(\mathbb{T})$ to $C^{2+\ell, \alpha}(\mathbb{T})$.

(c) For every fixed $\varphi \in \overline{B_{r_{K, 1}, C^{4+\ell, \alpha}}(0)}$, the map

$$
(P, R, \omega) \mapsto Y_{i, \varepsilon, P, R, \omega}[\varphi]: \quad M \times S O(3) \times K \rightarrow C^{2+\ell, \alpha}(\mathbb{T})
$$

is smooth. Moreover,

$$
\sum_{k=0}^{2}\left\|D_{P, R, \omega}^{k}\left(Y_{i, \varepsilon, P, R, \omega}[\varphi]-Y_{i, R, \omega}[\varphi]\right)\right\|_{C^{2+\ell, \alpha}(\mathbb{T})} \leq C_{K, \ell}\left(\varepsilon^{2}+\|\varphi\|_{C^{4+\ell, \alpha}(\mathbb{T})}\right) .
$$

Proof. We first remark that

$$
\begin{aligned}
g_{\varepsilon, P, R, \omega, \alpha \beta}(y) & =g_{\varepsilon, P}\left(R T_{\omega}(y)\right)\left[D\left(R T_{\omega}\right)(y)\left[\mathbf{e}_{\alpha}\right], D\left(R T_{\omega}\right)(y)\left[\mathbf{e}_{\beta}\right]\right], \\
g_{0, R, \omega}(y) & =g_{0}\left[D\left(R T_{\omega}\right)(y)\left[\mathbf{e}_{\alpha}\right], D\left(R T_{\omega}\right)(y)\left[\mathbf{e}_{\beta}\right]\right]
\end{aligned}
$$

where $\mathbf{e}_{\alpha}$ denotes the canonical basis in $\mathbb{R}^{3}$. Recalling (28) and (30), it is easily seen that for any $\ell \in \mathbb{N}$, we have

$$
\sum_{k=0}^{2}\left\|D_{P, R, \omega}^{k}\left(g_{\varepsilon, P, R, \omega, \alpha \beta}-g_{0, P, R, \omega, \alpha \beta}\right)\right\|_{C^{\ell}\left(\overline{B_{\rho_{K}}(0)}\right)} \leq C_{K, \ell} \varepsilon^{2}
$$


where $\rho_{K}$ appears in the proof of Lemma 3.3. This yields

$$
\sum_{k=0}^{2}\left\|D_{P, R, \omega}^{k}\left(n_{\varepsilon, P, R, \omega}-n_{0, R, \omega}\right)\right\|_{C^{\ell}(\mathbb{T})} \leq C_{K, \ell} \varepsilon^{2} .
$$

We define position vectors for $\Sigma_{\varepsilon, P, R, \omega}[\varphi]$ and $R \mathbb{T}_{\omega}[\varphi]$ in $\left(\mathbb{R}^{3}, g_{\varepsilon, P, R, \omega}\right)$ and $\left(\mathbb{R}^{3}, g_{0, R, \omega}\right)$ by

$$
\mathcal{Z}_{\varepsilon, P, R, \omega}[\varphi](p):=p+\varphi(p) n_{\varepsilon, P, R, \omega}(p), \quad \mathcal{Z}_{0, R, \omega}[\varphi](p):=p+\varphi(p) n_{0, R, \omega}(p) .
$$

Clearly, the map

$$
M \times S O(3) \times K \times C^{4+\ell, \alpha}(\mathbb{T}) \ni(P, R, \omega, \varphi) \mapsto\left(\mathcal{Z}_{\varepsilon, P, R, \omega}[\varphi], \mathcal{Z}_{0, R, \omega}[\varphi]\right) \in\left(C^{4+\ell, \alpha}\left(\mathbb{T}, \mathbb{R}^{3}\right)\right)^{2}
$$

is smooth. Hence, we also see the smoothness of maps

$$
\begin{aligned}
& (P, R, \omega, \varphi) \mapsto g_{\varepsilon, P, R, \omega, \alpha \beta}\left(\mathcal{Z}_{\varepsilon, P, R, \omega}[\varphi]\right): M \times S O(3) \times K \times \overline{B_{r_{K, \ell, 1}, C^{4+\ell, \alpha}}(0)} \rightarrow C^{4+\ell, \alpha}(\mathbb{T}, \mathbb{R}), \\
& (P, R, \omega, \varphi) \mapsto \bar{g}_{\varepsilon, P, R, \omega}[\varphi]: M \times S O(3) \times K \times \overline{B_{r_{K, \ell, 1}, C^{4+\ell, \alpha}}(0)} \rightarrow C^{3+\ell, \alpha}\left(\mathbb{T},(T \mathbb{T})^{*} \otimes(T \mathbb{T})^{*}\right), \\
& (P, R, \omega, \varphi) \mapsto n_{\varepsilon, P, R, \omega}[\varphi]: M \times S O(3) \times K \times \overline{B_{r_{K, \ell, 1}, C^{4+\ell, \alpha}}(0)} \rightarrow C^{3+\ell, \alpha}\left(\mathbb{T}, \mathbb{R}^{3}\right), \\
& (P, R, \omega, \varphi) \mapsto H_{\varepsilon, P, R, \omega}[\varphi]: M \times S O(3) \times K \times \overline{B_{r_{K, \ell, 1}, C^{4+\ell, \alpha}}(0)} \rightarrow C^{2+\ell, \alpha}(\mathbb{T})
\end{aligned}
$$

where $n_{\varepsilon, P, R, \omega}[\varphi]$ denotes the unit outer normal of $(\mathbb{T}[\varphi])_{\varepsilon, P, R, \omega}$ in the metric $g_{\varepsilon, P, R, \omega}$. From this fact, it is easily seen that the assertion (a) holds.

Moreover, by (37), there holds

$$
\begin{aligned}
& \sum_{k=0}^{2}\left\|D_{P, R, \omega}^{k}\left(\mathcal{Z}_{\varepsilon, P, R, \omega}[\varphi]-\mathcal{Z}_{0, R, \omega}[\varphi]\right)\right\|_{C^{4+\ell, \alpha}\left(\mathbb{T}, \mathbb{R}^{3}\right)} \leq C_{K, \ell}\|\varphi\|_{C^{4+\ell, \alpha}(\mathbb{T})} \varepsilon^{2}, \\
& \left\|D_{\varphi}\left(\mathcal{Z}_{\varepsilon, P, R, \omega}[\varphi]-\mathcal{Z}_{0, R, \omega}[\varphi]\right)\right\|_{\mathcal{L}\left(C^{4+\ell, \alpha}(\mathbb{T}), C^{4+\ell, \alpha}\left(\mathbb{T}, \mathbb{R}^{3}\right)\right)} \\
& \quad+\left\|D_{\varphi}^{2}\left(\mathcal{Z}_{\varepsilon, P, R, \omega}[\varphi]-\mathcal{Z}_{0, R, \omega}[\varphi]\right)\right\|_{\mathcal{L}^{2}\left(C^{4+\ell, \alpha}(\mathbb{T}), C^{4+\ell, \alpha}\left(\mathbb{T}, \mathbb{R}^{3}\right)\right)} \leq C_{K, \ell} \varepsilon^{2}
\end{aligned}
$$

Noting that similar estimates hold for $g_{\varepsilon, P, R, \omega, \alpha \beta}\left(\mathcal{Z}_{\varepsilon, P, R, \omega}[\varphi]\right), \bar{g}_{\varepsilon, P, R, \omega}[\varphi], n_{\varepsilon, P, R, \omega}[\varphi]$ and $H_{\varepsilon, P, R, \omega}[\varphi]$ thanks to (36), we observe that assertions (b) and (c) also hold.

We next proceed at a non-linear level, exploiting Proposition 2.11 and using the inverse mapping theorem.

Proposition 3.5. Fix a compact subset $K$ of $\mathbb{D}$ as above. Then there exist positive constants $\bar{C}_{K}$ and $0<\varepsilon_{K, 2} \leq \varepsilon_{K, 1,1}$ such that for any $\varepsilon \in\left(0, \varepsilon_{K, 2}\right]$ and $(P, R, \omega) \in M \times S O(3) \times K$, there exists a function $\varphi_{\varepsilon}=\varphi_{\varepsilon}(P, R, \omega) \in C^{5, \alpha}(\mathbb{T})$ satisfying the following:

$$
\begin{aligned}
& \left.j) \quad W_{g_{\varepsilon}}^{\prime}\left(\Sigma_{\varepsilon, P, R, \omega}\left[\varphi_{\varepsilon}\right]\right)=\beta_{0} H_{\varepsilon, P, R, \omega}\left[\varphi_{\varepsilon}\right]+\sum_{i=1}^{7} \beta_{i} Z_{i, R, \omega} ; \quad j j\right) \quad\left|\Sigma_{\varepsilon, P, R, \omega}\left[\varphi_{\varepsilon}\right]\right|_{g_{\varepsilon}}=4 \sqrt{2} \pi^{2} ; \\
& \left.j j j) \quad\left\langle Y_{j, \varepsilon, P, R, \omega}\left[\varphi_{\varepsilon}\right], \varphi_{\varepsilon}\right\rangle_{L_{\varepsilon, P, R, \omega, \varphi_{\varepsilon}}^{2}}=0 \quad(1 \leq j \leq 7) ; \quad j j j j\right) \quad\left\|\varphi_{\varepsilon}(P, R, \omega)\right\|_{C^{5, \alpha}(\mathbb{T})} \leq \bar{C}_{K} \varepsilon^{2}
\end{aligned}
$$

for some numbers $\beta_{0}, \ldots, \beta_{7}$. In particular, $\varphi_{\varepsilon}(P, R, \omega) \in \mathcal{M}_{\varepsilon, P, R, \omega}$. Moreover, for each $\varepsilon \in\left(0, \varepsilon_{K, 2}\right]$, the map

$$
M \times S O(3) \times K \rightarrow C^{5, \alpha}(\mathbb{T}): \quad(P, R, \omega) \mapsto \varphi_{\varepsilon}(P, R, \omega)
$$

is smooth and satisfies

$$
\left\|D_{P, R, \omega} \varphi_{\varepsilon}(P, R, \omega)\right\|_{C^{5, \alpha}(\mathbb{T})}+\left\|D_{P, R, \omega}^{2} \varphi_{\varepsilon}(P, R, \omega)\right\|_{C^{5, \alpha}(\mathbb{T})} \leq C_{K} \varepsilon^{2} .
$$


Proof. We first define $G_{\varepsilon}(P, R, \omega, \varphi): M \times S O(3) \times K \times C^{5, \alpha}(\mathbb{T}) \rightarrow C^{1, \alpha}(\mathbb{T})$ by

$$
\begin{aligned}
G_{\varepsilon}(P, R, \omega, \varphi):= & \Pi_{\varepsilon, P, R, \omega}^{\varphi}\left(W_{g_{\varepsilon}}^{\prime}\left(\Sigma_{\varepsilon, P, R, \omega}[\varphi]\right)\right)+\left(\left|\Sigma_{\varepsilon, P, R, \omega}[\varphi]\right|_{g_{\varepsilon}}-4 \sqrt{2} \pi^{2}\right) H_{\varepsilon, P, R, \omega}[\varphi] \\
& +\sum_{j=1}^{7}\left\langle Y_{j, \varepsilon, P, R, \omega}[\varphi], \varphi\right\rangle_{L_{\varepsilon, P, R, \omega, \varphi}^{2}} Y_{j, \varepsilon, P, R, \omega}[\varphi] .
\end{aligned}
$$

Since $\Pi_{\varepsilon, P, R, \omega}^{\varphi}$ is $L_{\varepsilon, P, R, \omega}^{2}(\mathbb{T})$-projection into $\left(\mathcal{K}_{\varepsilon, P, R, \varphi}^{\varphi}\right)^{\perp \bar{g}_{\varepsilon, P, R, \omega}[\varphi]}$ and $\mathcal{K}_{\varepsilon, P, R, \varphi}^{\varphi}$ is spanned by $H_{\varepsilon, P, R, \omega}[\varphi]$ and $Y_{j, \varepsilon, P, R, \omega}[\varphi](1 \leq j \leq 7)$, in order to prove $\left.\left.j\right), j j\right)$ and $\left.j j j\right)$, it is enough to find $\varphi \in C^{5, \alpha}(\mathbb{T})$ so that $G_{\varepsilon}(P, R, \omega, \varphi)=0$.

To this end, we first claim that there exist $r_{K, 2} \in\left(0, r_{K, 1,1}\right]$ and $\varepsilon_{K, 2} \in\left(0, \varepsilon_{K, 1,1}\right]$ such that $D_{\varphi} G_{\varepsilon}(P, R, \omega, \varphi)$ is invertible for every $(P, R, \omega, \varphi) \in M \times S O(3) \times K \times C^{5, \alpha}(\mathbb{T})$ provided $\|\varphi\|_{C^{5, \alpha}(\mathbb{T})} \leq r_{K, 2}$ and $0<\varepsilon \leq \varepsilon_{K, 2}$. We notice that $G_{\varepsilon}$ is smooth in each variable thanks to Lemma 3.4. Furthermore, by (36), (37) and (38), we observe that

$$
\begin{aligned}
& \sum_{k=0}^{2}\left\|D_{P, R, \omega}^{k}\left(G_{\varepsilon}(P, R, \omega, \varphi)-G_{0}(R, \omega, \varphi)\right)\right\|_{C^{1, \alpha}(\mathbb{T})} \leq C_{K}\left(\varepsilon^{2}+\|\varphi\|_{C^{5, \alpha}(\mathbb{T})}\right), \\
& \left\|D_{\varphi}\left(G_{\varepsilon}(P, R, \omega, \varphi)-G_{0}(R, \omega, \varphi)\right)\right\|_{\mathcal{L}\left(C^{5, \alpha}(\mathbb{T}), C^{1, \alpha}(\mathbb{T})\right.} \\
& \quad+\left\|D_{\varphi}^{2}\left(G_{\varepsilon}(P, R, \omega, \varphi)-G_{0}(R, \omega, \varphi)\right)\right\|_{\mathcal{L}^{2}\left(C^{5, \alpha}(\mathbb{T}), C^{1, \alpha}(\mathbb{T})\right.} \leq C_{K} \varepsilon^{2}
\end{aligned}
$$

for all $(P, R, \omega, \varphi) \in M \times S O(3) \times K \times C^{5, \alpha}(\mathbb{T})$ provided $\|\varphi\|_{C^{5, \alpha}(\mathbb{T})} \leq r_{K, 1,1}$ where $G_{0}(R, \omega, \varphi)$ is a corresponding map defined for the Euclidean metric:

$$
\begin{aligned}
G_{0}(R, \omega, \varphi):= & \Pi_{0, R, \omega}^{\varphi}\left(W_{g_{0}}^{\prime}\left(R \mathbb{T}_{\omega}[\varphi]\right)\right)+\left(\left|R \mathbb{T}_{\omega}[\varphi]\right|_{g_{0}}-4 \sqrt{2} \pi^{2}\right) H_{0, R, \omega}[\varphi] \\
& +\sum_{j=1}^{7}\left\langle Y_{j, R, \omega}[\varphi], \varphi\right\rangle_{L_{0, R, \omega, \varphi}^{2}} Y_{j, R, \omega}[\varphi] .
\end{aligned}
$$

From (39), it suffices to show that $D_{\varphi} G_{0}(R, \omega, 0)$ is invertible.

To see this, we remark that for $\psi \in C^{5, \alpha}(\mathbb{T})$ and $|t| \ll 1$, the surface $R \mathbb{T}_{\omega}[t \psi]$ is a perturbation in the normal direction. Hence, by $W_{g_{0}}^{\prime}\left(R \mathbb{T}_{0}\right)=0$ and Corollary 2.10, we obtain

$$
\left.D_{\varphi} W_{g_{0}}^{\prime}\left(R \mathbb{T}_{\omega}[\varphi]\right)\right|_{\varphi=0}[\psi]=\left.\frac{d}{d t} W_{g_{0}}^{\prime}\left(R \mathbb{T}_{\omega}[t \psi]\right)\right|_{t=0}=\tilde{L}_{0, R, \omega} \psi
$$

Moreover, by $\left|R \mathbb{T}_{\omega}\right|_{g_{0}}=4 \sqrt{2} \pi^{2}$, one has

$$
D_{\varphi} G_{0}(R, \omega, 0)[\psi]=\Pi_{0, R, \omega}^{0} \tilde{L}_{0, R, \omega} \psi+\left\langle H_{0, R, \omega}[0], \psi\right\rangle_{L_{0, R, \omega}^{2}} H_{0, R, \omega}[0]+\sum_{j=1}^{7}\left\langle Y_{j, R, \omega}[0], \psi\right\rangle_{L_{0, R, \omega}^{2}, Y_{j, R, \omega}[0] .}
$$

Now we show that $D_{\varphi} G_{0}(R, \omega, 0)$ is injective. Let $D_{\varphi} G_{0}(R, \omega, 0)[\psi]=0$. Then we have

$$
0=\Pi_{0, R, \omega}^{0} \tilde{L}_{0, R, \omega} \psi=\left\langle H_{0, R, \omega}[0], \psi\right\rangle_{L_{0, R, \omega}^{2}}=\left\langle Y_{j, R, \omega}[0], \psi\right\rangle_{L_{0, R, \omega}^{2}} \quad(1 \leq j \leq 7),
$$

which implies $\psi \in \mathcal{K}_{0, R, \omega}^{\perp \bar{g}_{0, R}, \omega}$. Thus by Proposition 2.11 and $\Pi_{0, R, \omega}^{0} \tilde{L}_{0, R, \omega} \psi=0$, one sees $\psi \equiv 0$ and we infer that $D_{\varphi} G_{0}(R, \omega, 0)$ is injective.

Next we prove that $D_{\varphi} G_{0}(R, \omega, 0)$ is surjective. Recall that $Y_{j, R, \omega}[0] \in \operatorname{Ker} \tilde{L}_{0, R, \omega}(1 \leq j \leq 7)$ and $\left\langle H_{0, R, \omega}[0], Y_{j, R, \omega}[0]\right\rangle_{L_{0, R, \omega}^{2}}=0(1 \leq j \leq 7)$. Therefore, setting $\psi_{j}:=Y_{j, R, \omega}[0] \in C^{\infty}(\mathbb{T})(1 \leq j \leq 7)$, we have $D_{\varphi} G_{0}(P, R, \omega, 0)\left[\psi_{j}\right]=Y_{j, R, \omega}[0]$. Thus to prove that $D_{\varphi} G_{0}(P, R, \omega, 0)$ is surjective, it is sufficient to show that the restricted map

(40)

$$
D_{\varphi} G_{0}(P, R, \omega, 0): C^{5, \alpha}(\mathbb{T}) \cap\left(\mathcal{K}_{0, R, \omega}^{\perp \bar{g}_{0, R, \omega}} \oplus \operatorname{span}\left\{H_{0, R, \omega}[0]\right\}\right) \rightarrow C^{1, \alpha}(\mathbb{T}) \cap\left(\mathcal{K}_{0, R, \omega}^{\perp \bar{g}_{0, R, \omega}} \oplus \operatorname{span}\left\{H_{0, R, \omega}[0]\right\}\right)
$$


is surjective. For $\psi \in \mathcal{K}_{0, R, \omega}^{\perp \bar{g}_{0,2}, \omega}$, it follows that $D_{\varphi} G_{0}(P, R, \omega, 0)[\psi]=\Pi_{0, R, \omega}^{0} \tilde{L}_{0, R, \omega} \psi$. Hence, we have

$$
\left.D_{\varphi} G_{0}(P, R, \omega, 0)\right|_{\mathcal{K}_{0, R, \omega}^{\perp \bar{g}_{0, R, \omega}}}=\Pi_{0, R, \omega}^{0} \tilde{L}_{0, R, \omega}: C^{5, \alpha}(\mathbb{T}) \cap\left(\mathcal{K}_{0, R, \omega}^{\perp \bar{g}_{0, R, \omega}}\right) \rightarrow C^{1, \alpha}(\mathbb{T}) \cap\left(\mathcal{K}_{0, R, \omega}^{\perp \bar{g}_{0, R, \omega}}\right)
$$

and shall show that the map in (41) is surjective. If this claim holds true, then the map in (40) is surjective. Indeed, since

$$
\left(\mathrm{Id}-\Pi_{0, R, \omega}^{0}\right)\left(D_{\varphi} G_{0}(P, R, \omega, 0)\left[H_{0, R, \omega}[0]\right]\right)=\left\|H_{0, R, \omega}[0]\right\|_{L_{0, R, \omega}^{2}}^{2} H_{0, R, \omega}[0] \neq 0
$$

combining with the surjectivity of the map in (41), we may observe that the map in (40) is surjective.

Now we show that the map in (41) is surjective. For this purpose, denote by $i_{0, R, \omega}$ the inclusion map $C^{5, \alpha}(\mathbb{T}) \cap\left(\mathcal{K}_{0, R, \omega}^{\perp \bar{g}_{0, R}, \omega} \oplus \operatorname{span}\left\{H_{0, R, \omega}[0]\right\}\right) \subset C^{5, \alpha}(\mathbb{T})$. Then the map $\Pi_{0, R, \omega}^{0} \tilde{L}_{0, R, \omega}$ is decomposed as follows: $\Pi_{0, R, \omega}^{0} \tilde{L}_{0, R, \omega}=\Pi_{0, R, \omega}^{0} \circ \tilde{L}_{0, R, \omega} \circ i_{0, R, \omega}$. Notice that maps $i_{0, R, \omega}$ and $\Pi_{0, R, \omega}^{0}: C^{1, \alpha}(\mathbb{T}) \rightarrow \mathcal{K}_{0, R, \omega}^{\perp, \bar{g}_{0, R, \omega}}$ are the Fredholm operators with indices -8 and 8 . Moreover, using the elliptic regularity and the Fredholm alternative, we also observe that $\tilde{L}_{0, R, \omega}: C^{5, \alpha}(\mathbb{T}) \rightarrow C^{1, \alpha}(\mathbb{T})$ is the Fredholm operator with index 0 . Hence, $\Pi_{0, R, \omega}^{0} \tilde{L}_{0, R, \omega}=\Pi_{0, R, \omega}^{0} \circ \tilde{L}_{0, R, \omega} \circ i_{0, R, \omega}$ is the Fredholm operator with index 0 . Since we already know that $\Pi_{0, R, \omega}^{0} \circ \tilde{L}_{0, R, \omega}$ is injective, one may observe that $\Pi_{0, R, \omega}^{0} \tilde{L}_{0, R, \omega}$ is surjective and we conclude that $D_{\varphi} G_{0}(P, R, \omega, 0)$ is invertible.

From (39), for some $\varepsilon_{K, 2} \in\left(0, \varepsilon_{K, 1,1}\right]$, the map $D_{\varphi} G_{\varepsilon}(P, R, \omega, 0)$ is invertible provided $0 \leq \varepsilon \leq \varepsilon_{K, 2}$. Thus by the inverse mapping theorem, we may find neighbourhoods $U_{1, \varepsilon, P, R, \omega} \subset C^{5, \alpha}(\mathbb{T})$ and $U_{2, \varepsilon, P, R, \omega} \subset$ $C^{1, \alpha}(\mathbb{T})$ of 0 and $G_{\varepsilon}(P, R, \omega, 0)$ such that $G_{\varepsilon}(P, R, \omega, \cdot): U_{1, \varepsilon, P, R, \omega} \rightarrow U_{2, \varepsilon, P, R, \omega}$ is diffeomorphism. Noting (39) and a proof of the inverse mapping theorem (for instance, see Lang [20, Theorem 3.1 in Chapter XVIII]), one may find $r_{K, 2} \in\left(0, r_{K, 1,1}\right]$ so that

$$
\overline{B_{r_{K, 2}, C^{5, \alpha}(\mathbb{T})}(0)} \subset U_{1, \varepsilon, P, R, \omega}, \quad \overline{B_{2 r_{K, 2}, C^{1, \alpha}(\mathbb{T})}\left(G_{\varepsilon}(P, R, \omega, 0)\right)} \subset U_{2, \varepsilon, P, R, \omega}
$$

for all $(\varepsilon, P, R, \omega) \in\left[0, \varepsilon_{K, 2}\right] \times M \times S O(3) \times K$. Since $\left\|G_{\varepsilon}(P, R, \omega, 0)\right\|_{C^{1, \alpha}} \leq C_{K} \varepsilon^{2}$ holds due to Lemma 3.3. shrink $\varepsilon_{K, 2}>0$ if necessary, we have

$$
\overline{B_{r_{K, 2}, C^{1, \alpha}(\mathbb{T})}(0)} \subset \overline{B_{2 r_{K, 2}, C^{1, \alpha}(\mathbb{T})}\left(G_{\varepsilon}(P, R, \omega, 0)\right)} \text { for all } \varepsilon \in\left[0, \varepsilon_{K, 2}\right],
$$

which means that one may find a $\varphi_{\varepsilon}(P, R, \omega)$ uniquely in $U_{1, \varepsilon, P, R, \omega}$ such that $G_{\varepsilon}\left(P, R, \omega, \varphi_{\varepsilon}(P, R, \omega)\right)=0$. Moreover, from $\left\|G_{\varepsilon}(P, R, \omega, 0)\right\|_{C^{1, \alpha}} \leq C_{K} \varepsilon^{2}$ it follows that $\left\|\varphi_{\varepsilon}(P, R, \omega)\right\|_{C^{5, \alpha}(\mathbb{T})} \leq C_{K} \varepsilon^{2}$. Thus assertions $j), j j), j j j), j j j j)$ hold.

The smoothness of $\varphi_{\varepsilon}(P, R, \omega)$ in $(P, R, \omega)$ follows from the smoothness of $\left.G_{\varepsilon}(P, R, \omega, \varphi), j j j j\right)$, the fact that $D_{\varphi} G_{\varepsilon}\left(P, R, \omega, \varphi_{\varepsilon}(P, R, \omega)\right)$ is invertible and the implicit function theorem. So we only need to prove the estimates for $D_{P, R, \omega} \varphi_{\varepsilon}$ and $D_{P, R, \omega}^{2} \varphi_{\varepsilon}$. We differentiate $G_{\varepsilon}\left(P, R, \omega, \varphi_{\varepsilon}(P, R, \omega)\right)=0$ in $(P, R, \omega)$ to obtain

$$
0=\left(D_{P, R, \omega} G_{\varepsilon}\right)\left(P, R, \omega, \varphi_{\varepsilon}\right)+\left(D_{\varphi} G_{\varepsilon}\right)\left(P, R, \omega, \varphi_{\varepsilon}\right)\left[D_{P, R, \omega} \varphi_{\varepsilon}\right] .
$$

Noting $G_{0}(P, R, \omega, 0)=0$ for all $(P, R, \omega) \in M \times S O(3) \times K$, it follows that

$$
D_{P, R, \omega} G_{0}(R, \omega, 0)=0=D_{P, R, \omega}^{2} G_{0}(R, \omega, 0) .
$$

Thus by (39), jjjj), (42) and the facts $D_{\varphi} G_{\varepsilon}\left(P, R, \omega, \varphi_{\varepsilon}\right): C^{5, \alpha}(\mathbb{T}) \rightarrow C^{1, \alpha}(\mathbb{T})$ is invertible and the norm of its inverse is uniformly bounded with respect to $\varepsilon, P, R, \omega$, we have

$$
\left\|D_{P, R, \omega} \varphi_{\varepsilon}\right\|_{C^{5, \alpha}(\mathbb{T})} \leq\left\|\left(D_{\varphi} G_{\varepsilon}\left(P, R, \omega, \varphi_{\varepsilon}\right)\right)^{-1}\right\|_{\mathcal{L}\left(C^{1, \alpha}(\mathbb{T}), C^{5, \alpha}(\mathbb{T})\right)}\left\|D_{P, R, \omega} G_{\varepsilon}\left(P, R, \omega, \varphi_{\varepsilon}\right)\right\|_{C^{1, \alpha}(\mathbb{T})} \leq C_{K} \varepsilon^{2}
$$

We differentiate (42) and get

$$
\begin{aligned}
0= & \left(D_{P, R, \omega}^{2} G_{\varepsilon}\right)\left(P, R, \omega, \varphi_{\varepsilon}\right)+2\left(D_{P, R, \omega} D_{\varphi} G_{\varepsilon}\right)\left(P, R, \omega, \varphi_{\varepsilon}\right)\left[D_{P, R, \omega} \varphi_{\varepsilon}\right] \\
& +\left(D_{\varphi}^{2} G_{\varepsilon}\right)\left(P, R, \omega, \varphi_{\varepsilon}\right)\left[\left(D_{P, R, \omega} \varphi_{\varepsilon}\right)^{2}\right]+\left(D_{\varphi} G_{\varepsilon}\right)\left(P, R, \omega, \varphi_{\varepsilon}\right)\left[D_{P, R, \omega}^{2} \varphi_{\varepsilon}\right] .
\end{aligned}
$$

Using the fact $\left\|D_{P, R, \omega} \varphi_{\varepsilon}\right\|_{C^{5, \alpha}(\mathbb{T})} \leq C_{K} \varepsilon^{2}$, in a similar way to above, we also have $\left\|D_{P, R, \omega}^{2} \varphi_{\varepsilon}\right\|_{C^{5, \alpha}(\mathbb{T})} \leq$ $C_{K} \varepsilon^{2}$. Thus we complete the proof. 
We can finally encode the variational structure of the problem by means of the following result.

Proposition 3.6. Let $K \subset \subset \mathbb{D}, \varepsilon_{K, 2}$ and $\varphi_{\varepsilon}(P, R, \omega)$ be as in Proposition 3.5, and for $\varepsilon \in\left(0, \varepsilon_{K, 2}\right]$ define the function $\Phi_{\varepsilon}: \mathcal{T}_{K, \varepsilon} \rightarrow \mathbb{R}$ by

$$
\Phi_{\varepsilon}(P, R, \omega):=W_{g_{\varepsilon}}\left(\Sigma_{\varepsilon, P, R, \omega}\left[\varphi_{\varepsilon}(P, R, \omega)\right]\right),
$$

where $\Sigma_{\varepsilon, P, R, \omega}\left[\varphi_{\varepsilon}(P, R, \omega)\right]$ was defined in (35). Then there exists $\varepsilon_{K, 3} \in\left(0, \varepsilon_{K, 2}\right]$ such that if $\varepsilon \in\left(0, \varepsilon_{K, 3}\right]$ and $\left(P_{\varepsilon}, R_{\varepsilon}, \omega_{\varepsilon}\right) \in M \times S O(3) \times K$ is a critical point of $\Phi_{\varepsilon}$, then $\Sigma_{\varepsilon, P_{\varepsilon}, R_{\varepsilon}, \omega_{\varepsilon}}\left[\varphi_{\varepsilon}\left(P_{\varepsilon}, R_{\varepsilon}, \omega_{\varepsilon}\right)\right]$ satisfies the area-constrained Willmore equation.

Proof. First of all, a criticality of $\Phi_{\varepsilon}$ in $P$ is independent of choices of the scales of $M$, namely $g$ and $g_{\varepsilon}$. Hence, in this proof, we adapt the rescaled metric $g_{\varepsilon}$ for the differential in $P$.

Let $P_{0} \in M$ and $\left(U_{0}, \Phi_{0}\right)$ be a normal coordinate centred at $P_{0}$ of $\left(M, g_{\varepsilon}\right)$. For $P \in U_{0}$ with $z=\Phi_{0}(P)$, the position vector for $\Sigma_{\varepsilon, P, R, \omega}[\varphi]$ in $\left(U_{0}, \Phi_{0}\right)$ is expressed as

$$
\widetilde{\mathcal{Z}}_{\varepsilon, P, R, \omega}[\varphi](p)=\mathcal{X}_{\varepsilon}\left(1 ; z, T_{\varepsilon}(z)\left(R T_{\omega}\left(\mathcal{Z}_{\varepsilon, P, R, \omega}[\varphi](p)\right)\right)\right)
$$

where $T_{\varepsilon}(z): \mathbb{R}^{3} \rightarrow \mathbb{R}^{3}$ is a linear transformation with $T_{\varepsilon}(0)=\operatorname{Id}$ and $\mathcal{X}_{\varepsilon}(t ; z, v)$ a solution of

$$
\frac{d^{2} \mathcal{X}_{\varepsilon}^{\alpha}}{d t^{2}}+\Gamma_{\varepsilon, \beta \gamma}^{\alpha}\left(\mathcal{X}_{\varepsilon}\right) \frac{d \mathcal{X}_{\varepsilon}^{\beta}}{d t} \frac{d \mathcal{X}_{\varepsilon}^{\gamma}}{d t}=0, \quad\left(\mathcal{X}_{\varepsilon}(0 ; z, v), \frac{d \mathcal{X}_{\varepsilon}}{d t}(0 ; z, v)\right)=(z, v) \in \mathbb{R}^{6}
$$

and $\Gamma_{\varepsilon, \beta \gamma}^{\alpha}$ stands for the Christoffel symbol of $\left(M, g_{\varepsilon}\right)$ in $\left(U_{0}, \Phi_{0}\right)$. Noting (28), one may find that

$$
\begin{aligned}
& \left\|D_{z}^{k_{1}+1} T_{\varepsilon}(z)\right\|_{L^{\infty}} \leq C_{k_{1}} \varepsilon^{2}, \quad\left\|\Gamma_{\varepsilon, \beta \gamma}^{\alpha}\right\|_{C^{k_{2}}} \leq C_{k_{2}} \varepsilon^{2}, \\
& \mathcal{X}_{\varepsilon}(1 ; z, v)=z+v+\widetilde{R}_{\varepsilon, 1}(z, v), \quad\left\|D_{z}^{k_{1}} D_{v}^{k_{2}} \widetilde{R}_{\varepsilon, 1}(z, v)\right\|_{L^{\infty}} \leq C_{k_{1}, k_{2}} \varepsilon^{2}
\end{aligned}
$$

for all $k_{1}, k_{2} \geq 0$. Hence,

$$
\widetilde{\mathcal{Z}}_{\varepsilon, P, R, \omega}[\varphi](p)=z+R T_{\omega}\left(\mathcal{Z}_{\varepsilon, P, R, \omega}[\varphi](p)\right)+\widetilde{R}_{\varepsilon, 2}\left(z, R T_{\omega}\left(\mathcal{Z}_{\varepsilon, P, R, \omega}[\varphi](p)\right)\right)
$$

where $\widetilde{R}_{\varepsilon, 2}(z, v)$ satisfies the same estimate to $\widetilde{R}_{\varepsilon, 1}(z, v)$.

Next, denote by $\widetilde{Z}_{0, R, \omega}(p):=R T_{\omega}\left(\mathcal{Z}_{0, R, \omega}[0](p)\right)$ and $\widetilde{n}_{0, R, \omega}(p)$ the position vector and the unit outer normal of $R \mathbb{T}_{\omega}$ in $\left(\mathbb{R}^{3}, g_{0}\right)$. We remark that the differential in $z$ corresponds to that in $P$ and

$$
Z_{m, R, \omega}(p)=g_{0}\left[D_{z^{m}}\left(z+\widetilde{Z}_{0, R, \omega}(p)\right), \widetilde{n}_{0, R, \omega}(p)\right], \quad Z_{j, R, \omega}(p)=g_{0}\left[D_{R, \omega}\left(z+\widetilde{Z}_{0, R, \omega}(p)\right), \widetilde{n}_{0, R, \omega}(p)\right]
$$

for $1 \leq m \leq 3$ and $4 \leq j \leq 7$. Recalling (37), (38), (44) and Proposition 3.5, we have

$$
\begin{aligned}
& \widetilde{n}_{\varepsilon, P, R, \omega}\left[\varphi_{\varepsilon}\right]-\widetilde{n}_{0, R, \omega}=O_{C^{4, \alpha}}\left(\varepsilon^{2}\right), \quad D_{z^{m}} \widetilde{\mathcal{Z}}_{\varepsilon, P, R, \omega}\left[\varphi_{\varepsilon}\right](p)=\mathbf{e}_{m}+O_{C^{4, \alpha}}\left(\varepsilon^{2}\right), \\
& D_{R, \omega}\left(\widetilde{\mathcal{Z}}_{\varepsilon, P, R, \omega}\left[\varphi_{\varepsilon}\right](p)-\widetilde{\mathcal{Z}}_{0, R, \omega}(p)\right)=O_{C^{4, \alpha}}\left(\varepsilon^{2}\right)
\end{aligned}
$$

where $\widetilde{n}_{\varepsilon, P, R, \omega}\left[\varphi_{\varepsilon}\right]$ stands for the outer unit normal of $\Sigma_{\varepsilon, P, R, \omega}\left[\varphi_{\varepsilon}\right]$ in $\left(U_{0}, \Phi_{0}\right)$ and $\left\|O_{C^{4, \alpha}}\left(\varepsilon^{2}\right)\right\|_{C^{4, \alpha}} \leq$ $C_{K} \varepsilon^{2}$. Thus putting

$$
\begin{aligned}
\psi_{\varepsilon, P, R, \omega, m}(p):=g_{\varepsilon, P_{0}}\left(\widetilde{\mathcal{Z}}_{\varepsilon, P, R, \omega}\left[\varphi_{\varepsilon}\right]\right)\left[D_{z^{m}} \widetilde{\mathcal{Z}}_{\varepsilon, P, R, \omega}\left[\varphi_{\varepsilon}\right], \widetilde{n}_{\varepsilon, P, R, \omega}\left[\varphi_{\varepsilon}\right]\right], \\
\psi_{\varepsilon, P, R, \omega, j}(p):=g_{\varepsilon, P_{0}}\left(\widetilde{\mathcal{Z}}_{\varepsilon, P, R, \omega}\left[\varphi_{\varepsilon}\right]\right)\left[D_{R, \omega} \widetilde{\mathcal{Z}}_{\varepsilon, P, R, \omega}\left[\varphi_{\varepsilon}\right], \widetilde{n}_{\varepsilon, P, R, \omega}\left[\varphi_{\varepsilon}\right]\right]
\end{aligned}
$$

for $1 \leq m \leq 3$ and $4 \leq j \leq 7$, we observe that

$$
\left\|\psi_{\varepsilon, P, R, \omega, i}-Z_{i, R, \omega}\right\|_{C^{4, \alpha}(\mathbb{T})} \leq C_{K} \varepsilon^{2}
$$


for all $1 \leq i \leq 7$.

Now we differentiate $j j)$ in Proposition 3.5 by $(z, R, \omega)$ to get

$$
0=\left\langle H_{\varepsilon, P, R, \omega}\left[\varphi_{\varepsilon}\right], \psi_{\varepsilon, P, R, \omega, i}\right\rangle_{L_{\varepsilon, P, R, \omega, \varphi_{\varepsilon}}^{2}} .
$$

Next we differentiate $\Phi_{\varepsilon}(P, R, \omega)$. Using $\left.j\right)$ and $\left.j j j j\right)$, one sees that

$$
\begin{aligned}
D_{z, R, \omega}\left(\Phi_{\varepsilon}(P, R, \omega)\right) & =\left\langle W_{g_{\varepsilon}}^{\prime}\left(\Sigma_{\varepsilon, P, R, \omega}\right)\left[\varphi_{\varepsilon}\right], \psi_{\varepsilon, P, R, \omega, i}\right\rangle_{L_{\varepsilon, P, R, \omega}^{2}}=\sum_{k=1}^{7} \beta_{k}\left\langle Z_{k, R, \omega}, \psi_{\varepsilon, P, R, \omega, i}\right\rangle_{L_{\varepsilon, P, R, \omega, \varphi_{\varepsilon}}^{2}} \\
& =\sum_{k=1}^{7} \beta_{k}\left(\left\langle Z_{k, R, \omega}, Z_{i, R, \omega}\right\rangle_{L_{0, R, \omega}^{2}}+O_{K}\left(\varepsilon^{2}\right)\right)=: \sum_{k=1}^{7} \beta_{k} A_{\varepsilon, P, R, \omega, i k}
\end{aligned}
$$

Since $Z_{1, R, \omega}, \ldots, Z_{7, R, \omega}$ are linearly independent, a matrix $A_{0, R, \omega}$ defined by $A_{0, R, \omega, i k}:=\left\langle Z_{k, R, \omega}, Z_{i, R, \omega}\right\rangle_{L_{0, R, \omega}^{2}}$ is invertible. Thus we may find $\varepsilon_{K, 3} \in\left(0, \varepsilon_{K, 2}\right]$ such that $A_{\varepsilon, P, R, \omega}$ is also invertible provided $0<\varepsilon \leq \varepsilon_{K, 3}$.

Let $\left(P_{\varepsilon}, R_{\varepsilon}, \omega_{\varepsilon}\right)$ be a critical point of $\Phi_{\varepsilon}$. Then by (45), one observes that

$$
0=\sum_{k=1}^{7} A_{\varepsilon, P_{\varepsilon}, R_{\varepsilon}, \omega_{\varepsilon}, i k} \beta_{k} \quad(1 \leq i \leq 7) .
$$

Since $A_{\varepsilon, P_{\varepsilon}, R_{\varepsilon}, \omega_{\varepsilon}}$ is invertible, we obtain $0=\beta_{1}=\cdots=\beta_{7}$. Hence, Proposition 3.6 holds.

Given Proposition 3.6, it is useful to understand the dependence of $\Phi_{\varepsilon}$ upon $\Sigma$. The next result gives a useful Taylor expansion of this quantity in terms of $\varepsilon$.

Proposition 3.7. Let $K \subset \mathbb{D}$ be a compact set. Then there exists a $C_{K}>0$ such that

$$
\left|\Phi_{\varepsilon}(P, R, \omega)-W_{g_{\varepsilon}}\left(\Sigma_{\varepsilon, P, R, \omega}[0]\right)\right| \leq C_{K} \varepsilon^{4}
$$

holds for every $(\varepsilon, P, R, \omega) \in\left(0, \varepsilon_{K, 3}\right] \times M \times S O(3) \times K$.

Proof. By definition of $\Phi_{\varepsilon}$ as in (43) and by the Taylor expansion of the Willmore functional up to second order in the perturbation $\varphi_{\varepsilon}$, we get

$$
\begin{aligned}
& \Phi_{\varepsilon}(P, R, \omega) \\
= & W_{g_{\varepsilon}}\left(\Sigma_{\varepsilon, P, R, \omega}[0]\right)+\left\langle W_{g_{\varepsilon}}^{\prime}\left(\Sigma_{\varepsilon, P, R, \omega}[0]\right), \varphi_{\varepsilon}\right\rangle_{L_{\varepsilon, P, R, \omega, 0}^{2}}+O\left(\left\|\varphi_{\varepsilon}(P, R, \omega)\right\|_{C^{4, \alpha}(\mathbb{T})}^{2}\right) .
\end{aligned}
$$

Observe that by Proposition 3.5 we have $\left\|\varphi_{\varepsilon}(P, R, \omega)\right\|_{C^{4, \alpha}(\mathbb{T})}^{2} \leq C_{K} \varepsilon^{4}$. Moreover, since $\Sigma_{\varepsilon, P, R, \omega}[0]$ is an approximate solution in the sense of Lemma 3.3. we have

$$
\left|\left\langle W_{g_{\varepsilon}}^{\prime}\left(\Sigma_{\varepsilon, P, R, \omega}[0]\right), \varphi_{\varepsilon}\right\rangle_{L_{\varepsilon, P, R, \omega, 0}^{2}}\right| \leq C_{K} \varepsilon^{4} .
$$

Thus proposition follows by combining the above estimates.

\section{Energy expansions}

The non-compactness of the Möbius group might create in general difficulties in extremising the function $\Phi_{\varepsilon}$ (which would amount to solving our problem by Proposition 3.6). In this section we will expand the Willmore energy on exponential maps of symmetric tori (in the notation of the previous section these are denoted as $\left.\Sigma_{\varepsilon, P, R, 0}[0]\right)$, and of tori which are degenerating to spheres $\left(\Sigma_{\varepsilon, P, R, \omega}[0]\right.$ with $|\omega|$ close to 1$)$. A comparison between these two energies, which involves some local curvature quantities, will indeed help to rule-out a blow-up behaviour. 


\subsection{Willmore energy of symmetric tori}

Here we calculate the expansion of the Willmore functional at small area symmetric Clifford tori embedded in $(M, g)$ through the exponential map for each $P \in M$. Before stating the next Proposition, we recall that $\mathbb{T} \subset \mathbb{R}^{3}$ stands for the symmetric Clifford torus with the axial vector $\mathbf{e}_{z}=(0,0,1)$. See the beginning of Subsection 2.1.

Proposition 4.1. As $\varepsilon \rightarrow 0$, the following expansion holds:

$$
\sup _{(P, R) \in M \times S O(3)}\left|W_{g_{\varepsilon}}\left(\Sigma_{\varepsilon, P, R, 0}[0]\right)-8 \pi^{2}-4 \sqrt{2} \pi^{2}\left\{\operatorname{Sc}_{P}-\operatorname{Ric}_{P}\left(R \mathbf{e}_{z}, R \mathbf{e}_{z}\right)\right\} \varepsilon^{2}\right| \leq C_{0} \varepsilon^{3} .
$$

The proof of the above proposition relies on two lemmas below. First of all, we linearise (with respect to $\left.g_{\varepsilon}\right)$ the Willmore energy of $\mathbb{T}$ at the Euclidean metric.

Lemma 4.2. Let $\Sigma \subset \mathbb{R}^{3}$ be an embedded smooth closed surface and $\left(g_{t}\right)_{\alpha \beta}:=\delta_{\alpha \beta}+$ th $h_{\alpha \beta}$ a perturbation of the Euclidean metric $g_{0}$. With a slight abuse of notation we will denote by $g_{t}$ both the metric on $M$ and its restriction to $\Sigma$. Denote the mean curvature of $\left(\Sigma, g_{t}\right)$ by $H(t)$ and set

$$
W(t):=\int_{\Sigma} H(t)^{2} d \sigma_{t}
$$

where $d \sigma_{t}$ is the volume element of $\left(\Sigma, g_{t}\right)$. Then as $t \rightarrow 0$, the following expansion holds:

$$
W(t)=W(0)+t \dot{W}(0)+O\left(t^{2}\right)
$$

where

$$
\begin{gathered}
\dot{W}(0)=\frac{1}{2} \int_{\Sigma}\left[2 \sum_{i=1}^{2}\left\langle\frac{\partial h}{\partial n} e_{i}, e_{i}\right\rangle H(0)-4 \sum_{i=1}^{2} e_{i}\left(h_{n i}\right) H(0)+4 \sum_{i, j=1}^{2} h_{n j}\left\langle\nabla_{e_{i}} e_{i}, e_{j}\right\rangle H(0)\right. \\
\left.-2 h_{n n} H^{2}(0)+H^{2}(0) \operatorname{tr}\left(h_{\mid T_{p} \Sigma}\right)\right] d \sigma_{0}(p) .
\end{gathered}
$$

Here $\left\{e_{1}, e_{2}\right\}$ is an orthonormal basis of $T_{p} \Sigma, n$ the unit outer normal, $h_{n i}:=\left\langle h n, e_{i}\right\rangle, h_{n n}:=\langle h n, n\rangle$, and $h_{\mid T_{p} \Sigma}$ is the induced $(2,0)$-tensor on $\Sigma$.

Before the proof of Lemma 4.2 we introduce some notations. First, we denote by $\nabla^{g_{t}}$ the Levi-Civita connection of $\left(\mathbb{R}^{3}, g_{t}\right)$. Using the metric $g_{t}$, for every $p \in \Sigma$, we choose $e_{1, t}(p), e_{2, t}(p)$ and $n_{t}(p)$ so that $\left\{e_{1, t}(p), e_{2, t}(p)\right\}$ is an orthonormal basis of $T_{p} \Sigma$ with respect to the metric $g_{t}$ and $n_{t}(p)$ the outer normal unit to $\Sigma$ at $p$ in the metric $g_{t}$. Moreover, we may assume that $e_{1, t}(p), e_{2, t}(p)$ and $n_{t}(p)$ are smooth in $p$ and $t, e_{1,0}=e_{1}, e_{2,0}=e_{2}$ and $n_{0}=n$. In addition, we denote by $\Gamma_{\lambda \mu}^{\kappa}(t)$ the Christoffel symbols of $\left(\mathbb{R}^{3}, g_{t}\right)$.

Proof. Since it is easy to see that $W(t)$ is smooth with respect to $t$, it is enough to show (477). We divide our arguments into several steps. First we recall the variation of the volume element (see [6], Proposition 1.186) $d \sigma_{t}$.

Step 1: There holds

$$
\left.\frac{d}{d t} d \sigma_{t}\right|_{t=0}=\frac{1}{2} \operatorname{tr}\left(\left.h\right|_{T_{p} \Sigma}\right) d \sigma_{0}(p)
$$

Next we calculate the variation of $n_{t}$.

Step 2: The following hold:

$$
\left(\dot{n}_{0}\right)_{i}:=g_{0}\left(\dot{n}_{0}, e_{i, 0}\right)=-h_{n i}=:-h\left(n_{0}, e_{i, 0}\right), \quad\left(\dot{n}_{0}\right)_{n}:=g_{0}\left(\dot{n}_{0}, n_{0}\right)=-\frac{1}{2} h_{n n}=:-\frac{1}{2} h\left(n_{0}, n_{0}\right) .
$$


Since $\left\{e_{1,0}, e_{2,0}, n_{0}\right\}$ forms an orthonormal basis of $\mathbb{R}^{3}$, we have

$$
\dot{n}_{0}=-h_{n 1} e_{1,0}-h_{n 2} e_{2,0}-\frac{1}{2} h_{n n} n_{0} .
$$

By definition, there holds

$$
1=g_{t}\left(n_{t}, n_{t}\right)=g_{0}\left(n_{t}, n_{t}\right)+\operatorname{th}\left(n_{t}, n_{t}\right), \quad 0=g_{t}\left(n_{t}, e_{i, t}\right)=g_{0}\left(n_{t}, e_{i, t}\right)+\operatorname{th}\left(n_{t}, e_{i, t}\right) .
$$

Differentiating these equalities with respect to $t$, we obtain

$$
0=2 g_{0}\left(\dot{n}_{0}, n_{0}\right)+h\left(n_{0}, n_{0}\right), \quad 0=g_{0}\left(\dot{n}_{0}, e_{i, 0}\right)+g_{0}\left(n_{0}, \dot{e}_{i, 0}\right)+h\left(n_{0}, e_{i, 0}\right) .
$$

Noting that $e_{i, t} \in T_{p} \Sigma$ and that $T_{p} \Sigma$ is invariant with respect to $t$, one obtains $\dot{e}_{i, 0} \in T_{p} \Sigma$. Recalling that $n_{0} \perp T_{p} \Sigma$ with respect to $g_{0}$, one sees $g_{0}\left(n_{0}, \dot{e}_{i, 0}\right)=0$. Combining this fact with (49), Step 2 holds.

Step 3: One has

$$
\dot{H}(0)=-\sum_{i=1}^{2} e_{i, 0}\left(h_{n i}\right)+\sum_{i, j=1}^{2} h_{n j} g_{0}\left(\nabla_{e_{i, 0}}^{g_{0}} e_{i, 0}, e_{j, 0}\right)-\frac{1}{2} h_{n n} H(0)+\frac{1}{2} \sum_{i=1}^{2} g_{0}\left(\frac{\partial h}{\partial n_{0}} e_{i, 0}, e_{i, 0}\right) .
$$

We fix $p \in \Sigma$ and calculate $\dot{H}(0)$ at $p$. Let $A_{t}$ be the second fundamental form of $\Sigma$ in $\left(\mathbb{R}^{3}, g_{t}\right)$, i.e.,

$$
A_{t}(X, Y):=g_{t}\left(\nabla_{X}^{g_{t}} n_{t}, Y\right)
$$

and set $A_{i j}:=A_{0}\left(e_{i, 0}, e_{j, 0}\right)$. Recalling that $\left\{e_{1, t}, e_{2, t}\right\}$ is an orthonormal basis of $T_{p} \Sigma$, we get

$$
H(t)=\sum_{i=1}^{2} A_{t}\left(e_{i, t}, e_{i, t}\right)=\sum_{i=1}^{2} g_{t}\left(\nabla_{e_{i, t}}^{g_{t}} n_{t}, e_{i, t}\right)=\sum_{i=1}^{2}\left\{g_{0}\left(\nabla_{e_{i, t}}^{g_{t}} n_{t}, e_{i, t}\right)+\operatorname{th}\left(\nabla_{e_{i, t}}^{g_{t}} n_{t}, e_{i, t}\right)\right\}
$$

and

$$
\dot{H}(0)=\sum_{i=1}^{2}\left\{\left.\frac{d}{d t} g_{0}\left(\nabla_{e_{i, t}}^{g_{t}} n_{t}, e_{i, t}\right)\right|_{t=0}+h\left(\nabla_{e_{i, 0}}^{g_{0}} n_{0}, e_{i, 0}\right)\right\} .
$$

Next we compute the second term in (50). Since

$$
\nabla_{e_{i, 0}}^{g_{0}} n_{0}=\sum_{j=1}^{2} A_{i j} e_{j, 0}
$$

we have

$$
\sum_{i=1}^{2} h\left(\nabla_{e_{i, 0}}^{g_{0}} n_{0}, e_{i, 0}\right)=\sum_{i, j=1}^{2} A_{i j} h\left(e_{j, 0}, e_{i, 0}\right)=\sum_{i, j=1}^{2} A_{i j} h_{i j} .
$$

Let us turn next to the first term in (50): let $\left(x^{1}, x^{2}, x^{3}\right)$ be a global chart of $\mathbb{R}^{3}$ with $g_{0}\left(\partial / \partial x^{\alpha}, \partial / \partial x^{\beta}\right)=$ $\delta_{\alpha \beta}$. Noting that

$$
\frac{\partial g_{\alpha \lambda, 0}}{\partial x^{\mu}}=0 \quad \text { for all } \alpha, \lambda, \mu, \quad \Gamma_{\lambda \mu}^{\kappa}(t)=\frac{1}{2} g_{t}^{\kappa \alpha}\left(\frac{\partial g_{\alpha \mu, t}}{\partial x^{\lambda}}-\frac{\partial g_{\lambda \mu, t}}{\partial x^{\alpha}}+\frac{\partial g_{\alpha \lambda, t}}{\partial x^{\mu}}\right),
$$

it follows that

$$
\begin{aligned}
\left.\frac{d}{d t} \Gamma_{\lambda \mu}^{\kappa}(t)\right|_{t=0} & =\left.\frac{1}{2} \frac{d g_{t}^{\kappa \alpha}}{d t}\right|_{t=0}\left(\frac{\partial g_{\alpha \mu, 0}}{\partial x^{\lambda}}-\frac{\partial g_{\lambda \mu, 0}}{\partial x^{\alpha}}+\frac{\partial g_{\alpha \lambda, 0}}{\partial x^{\mu}}\right)+\frac{1}{2} g_{0}^{\kappa \alpha}\left(\frac{\partial h_{\alpha \mu}}{\partial x^{\lambda}}-\frac{\partial h_{\lambda \mu}}{\partial x^{\alpha}}+\frac{\partial h_{\alpha \lambda}}{\partial x^{\mu}}\right) \\
& =\frac{1}{2} \delta^{\kappa \alpha}\left(\frac{\partial h_{\alpha \mu}}{\partial x^{\lambda}}-\frac{\partial h_{\lambda \mu}}{\partial x^{\alpha}}+\frac{\partial h_{\alpha \lambda}}{\partial x^{\mu}}\right) .
\end{aligned}
$$


Thus, writing

$$
e_{i, t}=e_{i}^{\kappa}(t) \frac{\partial}{\partial x^{\kappa}}, \quad n_{t}=n^{\kappa}(t) \frac{\partial}{\partial x^{\kappa}},
$$

we get

$$
\begin{aligned}
\left.\frac{d}{d t} \nabla_{e_{i, t}}^{g_{t}} n_{0, t}\right|_{t=0} & =\left(\dot{e}_{i}^{\lambda} \frac{\partial n^{\kappa}}{\partial x^{\lambda}}+e_{i}^{\lambda} \frac{\partial \dot{n}^{\kappa}}{\partial x^{\lambda}}+\dot{\Gamma}_{\lambda \mu}^{\kappa} e_{i}^{\lambda} n^{\mu}+\Gamma_{\lambda \mu}^{\kappa} \dot{e}_{i}^{\lambda} n^{\mu}+\Gamma_{\lambda \mu}^{\kappa} e_{i}^{\lambda} \dot{n}^{\mu}\right) \frac{\partial}{\partial x^{\kappa}} \\
& =\nabla_{\dot{e}_{i, 0}}^{g_{0}} n_{0}+\nabla_{e_{i, 0}}^{g_{0}} \dot{n}_{0}+\dot{\Gamma}_{\lambda \mu}^{\kappa} e_{i}^{\lambda} n^{\mu} \frac{\partial}{\partial x^{\kappa}}
\end{aligned}
$$

and

(53)

$$
\left.\frac{d}{d t} g_{0}\left(\nabla_{e_{i, t}}^{g_{t}} n_{t}, e_{i, t}\right)\right|_{t=0}=g_{0}\left(\nabla_{\dot{e}_{i, 0}}^{g_{0}} n_{0}, e_{i, 0}\right)+g_{0}\left(\nabla_{e_{i, 0}}^{g_{0}} \dot{n}_{0}, e_{i, 0}\right)+\dot{\Gamma}_{\lambda \mu}^{\kappa} e_{i}^{\lambda} n^{\mu} e_{i}^{\alpha} \delta_{\kappa \alpha}+g_{0}\left(\nabla_{e_{i, 0}}^{g_{0}} n_{0}, \dot{e}_{i, 0}\right) .
$$

By (52), we have

$$
\dot{\Gamma}_{\lambda \mu}^{\kappa} e_{i}^{\lambda} n^{\mu} e_{i}^{\alpha} \delta_{\kappa \alpha}=\frac{1}{2} \delta_{\kappa \alpha} \delta^{\kappa \beta}\left(\frac{\partial h_{\beta \mu}}{\partial x^{\lambda}}-\frac{\partial h_{\lambda \mu}}{\partial x^{\beta}}+\frac{\partial h_{\beta \lambda}}{\partial x^{\mu}}\right) e_{i}^{\lambda} n^{\mu} e_{i}^{\alpha}=\frac{1}{2}\left(\frac{\partial h_{\beta \mu}}{\partial x^{\lambda}}-\frac{\partial h_{\lambda \mu}}{\partial x^{\beta}}+\frac{\partial h_{\beta \lambda}}{\partial x^{\mu}}\right) e_{i}^{\lambda} n^{\mu} e_{i}^{\beta} .
$$

Noting that, by a relabelling of the indices

$$
\frac{\partial h_{\beta \mu}}{\partial x^{\lambda}} e_{i}^{\lambda} n^{\mu} e_{i}^{\beta}=\frac{\partial h_{\lambda \mu}}{\partial x^{\beta}} e_{i}^{\lambda} n^{\mu} e_{i}^{\beta}
$$

we obtain

$$
\sum_{i=1}^{2} \dot{\Gamma}_{\lambda \mu}^{\kappa} e_{i}^{\lambda} n^{\mu} e_{i}^{\alpha} \delta_{\kappa \alpha}=\sum_{i=1}^{2} \frac{1}{2} g_{0}\left(\frac{\partial h}{\partial n_{0}} e_{i, 0}, e_{i, 0}\right) .
$$

We consider now $g_{0}\left(\nabla_{e_{i, 0}}^{g_{0}} \dot{n}_{0}, e_{i, 0}\right)$ : recalling (48), one has

$$
\begin{aligned}
g_{0}\left(\nabla_{e_{i, 0}}^{g_{0}} \dot{n}_{0}, e_{i, 0}\right)= & g_{0}\left(\nabla_{e_{i, 0}}^{g_{0}}\left\{\sum_{j=1}^{2}\left(-h_{n j} e_{j, 0}\right)-\frac{1}{2} h_{n n} n_{0}\right\}, e_{i, 0}\right) \\
= & -\sum_{j=1}^{2}\left[e_{i, 0}\left\{g_{0}\left(h_{n j} e_{j, 0}, e_{i, 0}\right)\right\}-g_{0}\left(h_{n j} e_{j, 0}, \nabla_{e_{i, 0}}^{g_{0}} e_{i, 0}\right)\right] \\
& -\left[\frac{1}{2} g_{0}\left(e_{i, 0}\left(h_{n n}\right) n_{0}, e_{i, 0}\right)+\frac{1}{2} h_{n n} g_{0}\left(\nabla_{e_{i, 0}}^{g_{0}} n_{0}, e_{i, 0}\right)\right] .
\end{aligned}
$$

By $g_{0}\left(e_{j, 0}, e_{i, 0}\right)=\delta_{j i}$ and $g_{0}\left(e_{i, 0}, n_{0}\right)=0$, there holds

$$
\begin{aligned}
g_{0}\left(\nabla_{e_{i, 0}}^{g_{0}} \dot{n}_{0}, e_{i, 0}\right) & =-\sum_{j=1}^{2} e_{i, 0}\left(h_{n j} \delta_{i j}\right)+\sum_{j=1}^{2} h_{n j} g_{0}\left(e_{j, 0}, \nabla_{e_{i, 0}}^{g_{0}} e_{i, 0}\right)-\frac{1}{2} h_{n n} g_{0}\left(\nabla_{e_{i, 0}}^{g_{0}} n_{0}, e_{i, 0}\right) \\
& =-e_{i, 0}\left(h_{n i}\right)+\sum_{j=1}^{2} h_{n j} g_{0}\left(e_{j, 0}, \nabla_{e_{i, 0}}^{g_{0}} e_{i, 0}\right)-\frac{1}{2} h_{n n} g_{0}\left(\nabla_{e_{i, 0}}^{g_{0}} n_{0}, e_{i, 0}\right) .
\end{aligned}
$$

Noting $H(0)=\sum_{k=1}^{2} g_{0}\left(\nabla_{e_{k, 0}}^{g_{0}} n_{0}, e_{k, 0}\right)$, we see

$$
\sum_{i=1}^{2} g_{0}\left(\nabla_{e_{i, 0}}^{g_{0}} \dot{n}_{0}, e_{i, 0}\right)=-\sum_{i=1}^{2} e_{i, 0}\left(h_{n i}\right)+\sum_{i, j=1}^{2} h_{n j} g_{0}\left(e_{j, 0}, \nabla_{e_{i, 0}}^{g_{0}} e_{i, 0}\right)-\frac{h_{n n}}{2} H(0) .
$$

Next, we calculate the terms

$$
\sum_{i=1}^{2} g_{0}\left(\nabla_{\dot{e}_{i, 0}}^{g_{0}} n_{0}, e_{i, 0}\right), \quad \sum_{i=1}^{2} g_{0}\left(\nabla_{e_{i, 0}}^{g_{0}} n_{0}, \dot{e}_{i, 0}\right) .
$$


For this purpose, we first compute $\dot{e}_{i, 0}$. Differentiating

$$
1=g_{t}\left(e_{i, t}, e_{i, t}\right)=g_{0}\left(e_{i, t}, e_{i, t}\right)+\operatorname{th}\left(e_{i, t}, e_{i, t}\right), \quad 0=g_{t}\left(e_{1, t}, e_{2, t}\right)=g_{0}\left(e_{1, t}, e_{2, t}\right)+\operatorname{th}\left(e_{1, t}, e_{2, t}\right),
$$

one observes

$$
0=2 g_{0}\left(\dot{e}_{i, 0}, e_{i, 0}\right)+h\left(e_{i, 0}, e_{i, 0}\right), \quad 0=g_{0}\left(\dot{e}_{1,0}, e_{2,0}\right)+g_{0}\left(e_{1,0}, \dot{e}_{2,0}\right)+h\left(e_{1,0}, e_{2,0}\right),
$$

which is expressed as

$$
\left(\dot{e}_{i, 0}\right)_{i}=-\frac{1}{2} h_{i i}, \quad\left(\dot{e}_{1,0}\right)_{2}+\left(\dot{e}_{2,0}\right)_{1}=-h_{12} .
$$

Set $\dot{e}_{1,0}=\alpha_{11} e_{1,0}+\alpha_{12} e_{2,0}$ and $\dot{e}_{2,0}=\alpha_{21} e_{1,0}+\alpha_{22} e_{2,0}$ : then we see that $\alpha_{i i}=-h_{i i} / 2$ and

$$
\sum_{i=1}^{2} g_{0}\left(\nabla_{\dot{e}_{i, 0}}^{g_{0}} n_{0}, e_{i, 0}\right)=\sum_{i=1}^{2} g_{0}\left(\nabla_{\sum_{j=1}^{2} \alpha_{i j} e_{j, 0}}^{g_{0}} n_{0}, e_{i, 0}\right)=\sum_{i, j=1}^{2} \alpha_{i j} g_{0}\left(\nabla_{e_{j, 0}}^{g_{0}} n_{0}, e_{i, 0}\right)=\sum_{i, j=1}^{2} \alpha_{i j} A_{j i} .
$$

Noting that $A_{i j}=A_{j i}$, we have

$$
\alpha_{12} A_{21}+\alpha_{21} A_{12}=A_{12}\left(\alpha_{12}+\alpha_{21}\right)=-A_{12} h_{12}=-\frac{1}{2} h_{12} A_{12}-\frac{1}{2} h_{21} A_{21} .
$$

Hence, it follows that

$$
\sum_{i=1}^{2} g_{0}\left(\nabla_{\dot{e}_{i, 0}}^{g_{0}} n_{0}, e_{i, 0}\right)=-\frac{1}{2} \sum_{i, j=1}^{2} A_{i j} h_{i j}
$$

Similarly we have

$$
\sum_{i=1}^{2} g_{0}\left(\nabla_{e_{i, 0}}^{g_{0}} n_{0}, \dot{e}_{i, 0}\right)=\sum_{i, j=1}^{2} g_{0}\left(\nabla_{e_{i, 0}}^{g_{0}} n_{0}, \alpha_{i j} e_{j, 0}\right)=\sum_{i, j=1}^{2} \alpha_{i j} A_{i j}=-\frac{1}{2} \sum_{i, j=1}^{2} A_{i j} h_{i j} .
$$

By (50)-(57), we obtain

$$
\dot{H}(0)=-\sum_{i=1}^{2} e_{i, 0}\left(h_{n i}\right)+\sum_{i, j=1}^{2} h_{n j} g_{0}\left(\nabla_{e_{i, 0}}^{g_{0}} e_{i, 0}, e_{j, 0}\right)-\frac{1}{2} h_{n n} H(0)+\frac{1}{2} \sum_{i=1}^{2} g_{0}\left(\frac{\partial h}{\partial n_{0}} e_{i, 0}, e_{i, 0}\right)
$$

and Step 3 is completed.

\section{Step 4: Conclusion}

Finally, we calculate $\dot{W}(0)$. Since there holds

$$
\dot{W}(0)=2 \int_{\Sigma} H(0) \dot{H}(0) d \sigma_{0}+\int_{\Sigma} H^{2}(0) d \dot{\sigma_{0}},
$$

steps 1 and 3 yield

$$
\begin{gathered}
\dot{W}(0)=\frac{1}{2} \int_{\Sigma}\left[-4 \sum_{i=1}^{2} e_{i, 0}\left(h_{n i}\right) H(0)+4 \sum_{i, j=1}^{2} h_{n j}\left\langle\nabla_{e_{i, 0}}^{g_{0}}, e_{j, 0}\right\rangle H(0)-2 h_{n n} H^{2}(0)\right. \\
\left.+2 \sum_{i=1}^{2}\left\langle\frac{\partial h}{\partial n_{0}} e_{i, 0}, e_{i, 0}\right\rangle H(0)+H^{2}(0) \operatorname{tr}\left(\left.h\right|_{T_{p} \Sigma}\right)\right] \mathrm{d} \sigma_{0} .
\end{gathered}
$$

Thus we completed the proof. 
Now we illustrate how we apply Lemma 4.2 to prove Proposition 4.1. Set $t:=\varepsilon^{2}$. Recalling (26) and the definition of $g_{\varepsilon}$, we obtain

$$
\left(g_{t}\right)_{\alpha \beta}(x)=\delta_{\alpha \beta}+\frac{t}{3} R_{\alpha \mu \nu \beta} x^{\mu} x^{\nu}+\tilde{R}(t, x), \quad\left|\left(\frac{\partial}{\partial x}\right)^{\alpha} \tilde{R}(t, x)\right| \leq C_{|\alpha|} t^{3 / 2}
$$

for each $|x| \leq t^{-1 / 2} \rho_{0}, \alpha \in \mathbb{Z}_{+}^{3}$ and all sufficiently small $t>0$.

Next, we set $\Sigma=R \mathbb{T}$ and

$$
h_{\alpha \beta}(x):=\frac{1}{3} R_{\alpha \mu \nu \beta} x^{\mu} x^{\nu}, \quad\left(\tilde{g}_{t}\right)_{\alpha \beta}(x):=\delta_{\alpha \beta}+t h_{\alpha \beta}(x) .
$$

We also define

$$
\tilde{W}(t):=\int_{R \mathbb{T}} \tilde{H}^{2}(t) d \sigma, \quad W(t):=W_{g_{t}}\left(\exp _{P}(R \mathbb{T})\right),
$$

where $\tilde{H}(t)$ denotes the mean curvature of $R \mathbb{T}$ in $\left(\mathbb{R}^{3}, \tilde{g}_{t}\right)$. Then from $(58)$ it follows that

$$
\left\|g_{t}-\tilde{g}_{t}\right\|_{C^{k}\left(\overline{B_{3}(0)}\right)} \leq C_{k} t^{3 / 2}
$$

for all $k \in \mathbb{N}$. Hence, we may find $C_{0}>0$, which is independent of $R \in S O(3)$, so that

$$
|W(t)-\tilde{W}(t)| \leq C_{0} t^{3 / 2} .
$$

Noting that $t=\varepsilon^{2}$, to prove Proposition 4.1, it suffices to show

$$
\dot{\tilde{W}}(0)=-4 \sqrt{2} \pi^{2}\left\{\operatorname{Sc}_{P}-\operatorname{Ric}_{P}\left(\operatorname{Re}_{z}, R \mathbf{e}_{z}\right)\right\} .
$$

Furthermore, using the orthonormal basis $\left\{R \mathbf{e}_{x}, R \mathbf{e}_{y}, R \mathbf{e}_{z}\right\}$ of $\mathbb{R}^{3}$, we can reduce ourselves to the case $\Sigma=\mathbb{T}$ (namely $R=\mathrm{Id}$ ) and it suffices to prove

$$
\dot{\tilde{W}}(0)=-4 \sqrt{2} \pi^{2}\left\{\operatorname{Sc}_{P}-\operatorname{Ric}_{P}\left(\mathbf{e}_{z}, \mathbf{e}_{z}\right)\right\}=-4 \sqrt{2} \pi^{2}\left\{R_{11}+R_{22}\right\} .
$$

Now we will apply Lemma 4.2 to obtain (60). We use the map $X$ defined in (5) for parametrizing $\mathbb{T}$, and as $e_{1}, e_{2}$ and $n$ we choose

$$
\begin{aligned}
e_{1} & :=X_{\varphi}=(-\sin \varphi \cos \theta,-\sin \varphi \sin \theta, \cos \varphi), \\
e_{2} & :=X_{\theta} /\left|X_{\theta}\right|=(-\sin \theta, \cos \theta, 0), \\
n & :=(\cos \varphi \cos \theta, \cos \varphi \sin \theta, \sin \varphi) .
\end{aligned}
$$

Lemma 4.3. The following hold:

(I) The volume element $d \sigma$ and the mean curvature of $\mathbb{T}$ with respect to the Euclidean metric are

$$
d \sigma=(\sqrt{2}+\cos \varphi) d \varphi d \theta \quad \text { and } \quad H=\frac{\sqrt{2}+2 \cos \varphi}{\sqrt{2}+\cos \varphi} .
$$

(II) $\left\langle\nabla_{e_{1}} e_{1}, e_{1}\right\rangle=\left\langle\nabla_{e_{2}} e_{2}, e_{2}\right\rangle=\left\langle\nabla_{e_{1}} e_{1}, e_{2}\right\rangle=0$ and $\left\langle\nabla_{e_{2}} e_{2}, e_{1}\right\rangle=\sin \varphi /(\sqrt{2}+\cos \varphi)$.

(III) For the Ricci tensor $\operatorname{Ric}_{P}$, one has

$$
\begin{aligned}
& \int_{0}^{2 \pi} \operatorname{Ric}_{P}(X, n) d \theta=\pi\left[\operatorname{Sc}_{P}(\sqrt{2}+\cos \varphi) \cos \varphi+R_{33}\left\{2 \sin ^{2} \varphi-(\sqrt{2}+\cos \varphi) \cos \varphi\right\}\right] \\
& \int_{0}^{2 \pi} \operatorname{Ric}_{P}(n, n) d \theta=\pi\left[\operatorname{Sc}_{P} \cos ^{2} \varphi+R_{33}\left(2-3 \cos ^{2} \varphi\right)\right] \\
& \int_{0}^{2 \pi} \operatorname{Ric}_{P}\left(X, e_{1}\right) d \theta=\pi\left[-\operatorname{Sc}_{P}(\sqrt{2}+\cos \varphi) \sin \varphi+R_{33}\{\sqrt{2} \sin \varphi+3 \cos \varphi \sin \varphi\}\right], \\
& \int_{0}^{2 \pi} \operatorname{Ric}_{P}\left(n, e_{1}\right) d \theta=\pi\left[-\operatorname{Sc}_{P} \cos \varphi \sin \varphi+3 R_{33} \cos \varphi \sin \varphi\right] .
\end{aligned}
$$


Here we remark that $X, n, e_{1}$ are expressed in normal coordinates around $P \in M$ :

$X=X^{\alpha}\left(\frac{\partial}{\partial x^{\alpha}}\right)_{P}, n=n^{\alpha}\left(\frac{\partial}{\partial x^{\alpha}}\right)_{P}, e_{1}=e_{1}^{\alpha}\left(\frac{\partial}{\partial x^{\alpha}}\right)_{P} \in T_{P} M, \quad g(P)\left(\left(\frac{\partial}{\partial x^{\alpha}}\right)_{P},\left(\frac{\partial}{\partial x^{\beta}}\right)_{P}\right)=\delta_{\alpha \beta}$.

(IV) There holds

$$
\int_{0}^{2 \pi} \frac{1}{\sqrt{2}+\cos \varphi} d \varphi=2 \pi, \quad \int_{0}^{2 \pi} \frac{\cos \varphi}{\sqrt{2}+\cos \varphi} d \varphi=2 \pi-2 \sqrt{2} \pi, \quad \int_{0}^{2 \pi} \frac{\cos ^{2} \varphi}{\sqrt{2}+\cos \varphi} d \varphi=4 \pi-2 \sqrt{2} \pi .
$$

Proof. Assertions (I) and (II) are basic computations. For (III), note that

$$
\operatorname{Ric}_{P}(X, n)=R_{\alpha \beta} X^{\alpha} n^{\beta}, \quad \operatorname{Sc}_{P}=R_{11}+R_{22}+R_{33} .
$$

Since

$$
0=\int_{0}^{2 \pi} \cos \theta d \theta=\int_{0}^{2 \pi} \sin \theta d \theta=\int_{0}^{2 \pi} \sin \theta \cos \theta d \theta, \quad \pi=\int_{0}^{2 \pi} \cos ^{2} \theta d \theta=\int_{0}^{2 \pi} \sin ^{2} \theta d \theta,
$$

it follows from the definitions of $X, n$ and (61) that

$$
\begin{aligned}
\int_{0}^{2 \pi} \operatorname{Ric}_{P}(X, n) d \theta & =R_{11} \pi(\sqrt{2}+\cos \varphi) \cos \varphi+R_{22} \pi(\sqrt{2}+\cos \varphi) \cos \varphi+2 \pi R_{33} \sin ^{2} \varphi \\
& =\pi\left[\operatorname{Sc}_{P}(\sqrt{2}+\cos \varphi) \cos \varphi+R_{33}\left\{2 \sin ^{2} \varphi-(\sqrt{2}+\cos \varphi) \cos \varphi\right\}\right] .
\end{aligned}
$$

We can show in a similar way for other quantities, so we omit the details.

Finally, for (IV), using the change of variables

$$
t=\tan \left(\frac{\varphi}{2}\right), \quad \cos \varphi=\frac{1-t^{2}}{1+t^{2}}, \quad d \varphi=\frac{2}{1+t^{2}} d t \quad \text { for } \varphi \in(0, \pi)
$$

and

$$
\cos \varphi=(\sqrt{2}+\cos \varphi)-\sqrt{2}, \quad \cos ^{2} \varphi=(\cos \varphi+\sqrt{2})(\cos \varphi-\sqrt{2})+2,
$$

we easily get (IV). Thus we complete the proof.

We will now prove Proposition 4.1

Proof of Proposition 4.1. By the above arguments, it is enough to prove (60). First we express $h$ in (59) in terms of $\operatorname{Sc}_{P}$ and $\operatorname{Ric}_{P}$. Substituting $g_{\alpha \beta}=\delta_{\alpha \beta}$ and (27) into (59), we get

$$
h_{\alpha \beta}(x)=\frac{\operatorname{Sc}_{P}}{6}\left(|x|^{2} \delta_{\alpha \beta}-x_{\alpha} x_{\beta}\right)-\frac{1}{3} \delta_{\alpha \beta} \operatorname{Ric}_{P}(x, x)-\frac{1}{3}|x|^{2} R_{\alpha \beta}+\frac{1}{3}\left(x_{\alpha} R_{\beta \mu} x^{\mu}+x_{\beta} R_{\alpha \mu} x^{\mu}\right) .
$$

For the normal derivative of $h$, it follows from (62) that

$$
\begin{aligned}
\frac{\partial h_{\alpha \beta}}{\partial n}(x)= & \left.\frac{d}{d t} h_{\alpha \beta}(x+t n)\right|_{t=0} \\
= & \frac{\operatorname{Sc}_{P}}{6}\left(2\langle x, n\rangle \delta_{\alpha \beta}-x_{\alpha} n_{\beta}-n_{\alpha} x_{\beta}\right)-\frac{2}{3} \delta_{\alpha \beta} \operatorname{Ric}_{P}(x, n)-\frac{2}{3}\langle x, n\rangle R_{\alpha \beta} \\
& +\frac{1}{3}\left(x_{\alpha} R_{\beta \mu} n^{\mu}+n_{\alpha} R_{\beta \mu} x^{\mu}+x_{\beta} R_{\alpha \mu} n^{\mu}+n_{\beta} R_{\alpha \mu} x^{\mu}\right) .
\end{aligned}
$$

In what follows, we compute each term in (47) using Lemma 4.3, (62) and (63).

Step 1: $2 \sum_{i=1}^{2}\left\langle\frac{\partial h}{\partial n} e_{i}, e_{i}\right\rangle H$ 
Since it follows from (63) that

$$
\begin{aligned}
\operatorname{tr}_{\mathbb{R}^{3}}\left(\frac{\partial h}{\partial n}(X)\right)= & \frac{\operatorname{Sc}_{P}}{6}\{6\langle X, n\rangle-2\langle X, n\rangle\}-2 \operatorname{Ric}_{P}(X, n)-\frac{2}{3}\langle X, n\rangle \operatorname{Sc}_{P}+\frac{4}{3} \operatorname{Ric}_{P}(X, n) \\
= & -\frac{2}{3} \operatorname{Ric}_{P}(X, n), \\
\left\langle\frac{\partial h}{\partial n} n, n\right\rangle= & \frac{\operatorname{Sc}_{P}}{6}\{2\langle X, n\rangle-2\langle X, n\rangle\}-\frac{2}{3} \operatorname{Ric}_{P}(X, n)-\frac{2}{3}\langle X, n\rangle \operatorname{Ric}_{P}(n, n) \\
& +\frac{2}{3}\left\{\langle X, n\rangle \operatorname{Ric}_{P}(n, n)+\operatorname{Ric}_{P}(X, n)\right\}=0,
\end{aligned}
$$

we obtain

$$
\sum_{i=1}^{2}\left\langle\frac{\partial h}{\partial n}(X) e_{i}, e_{i}\right\rangle=-\frac{2}{3} \operatorname{Ric}_{P}(X, n)
$$

Therefore, by Lemma 4.3, we have

$$
\begin{aligned}
& \int_{\mathbb{T}} 2 \sum_{i=1}^{2}\left\langle\frac{\partial h}{\partial n}(X) e_{i}, e_{i}\right\rangle H d \sigma_{0} \\
= & -\frac{4}{3} \int_{0}^{2 \pi} \int_{0}^{2 \pi} \operatorname{Ric}_{P}(X, n)(\sqrt{2}+2 \cos \varphi) d \theta d \varphi \\
= & -\frac{4 \pi}{3} \int_{0}^{2 \pi}\left[\operatorname{Sc}_{P}(\sqrt{2}+\cos \varphi) \cos \varphi+R_{33}\left\{2 \sin ^{2} \varphi-(\sqrt{2}+\cos \varphi) \cos \varphi\right\}\right](\sqrt{2}+2 \cos \varphi) d \varphi
\end{aligned}
$$

Since

$$
\begin{aligned}
\int_{0}^{2 \pi}(\sqrt{2}+\cos \varphi)(\sqrt{2}+2 \cos \varphi) \cos \varphi d \varphi & =\int_{0}^{2 \pi}\left(2+3 \sqrt{2} \cos \varphi+2 \cos ^{2} \varphi\right) \cos \varphi d \varphi=3 \sqrt{2} \pi \\
\int_{0}^{2 \pi} 2 \sin ^{2} \varphi(\sqrt{2}+2 \cos \varphi) d \varphi & =\int_{0}^{2 \pi} 2 \sqrt{2} \sin ^{2} \varphi+4 \sin ^{2} \varphi \cos \varphi d \varphi=2 \sqrt{2} \pi
\end{aligned}
$$

we get

$$
\begin{aligned}
\int_{\mathbb{T}} 2 \sum_{i=1}^{2}\left\langle\frac{\partial h}{\partial n}(X) e_{i}, e_{i}\right\rangle H d \sigma_{0} & =-\frac{4 \pi}{3}\left\{3 \sqrt{2} \pi \operatorname{Sc}_{P}+R_{33}(2 \sqrt{2} \pi-3 \sqrt{2} \pi)\right\} \\
& =-4 \sqrt{2} \pi^{2} \operatorname{Sc}_{P}+\frac{4 \sqrt{2}}{3} \pi^{2} R_{33} .
\end{aligned}
$$

Step 2: $-4 \sum_{i=1}^{2} e_{i}\left(h_{n i}\right) H+4 \sum_{i, j=1}^{2} h_{n j}\left\langle\nabla_{e_{i}} e_{i}, e_{j}\right\rangle H$.

For a smooth function $f \in C^{\infty}(\Sigma)$, we have

$$
e_{1}(f)=\frac{\partial f}{\partial \varphi}, \quad e_{2}(f)=\frac{1}{\sqrt{2}+\cos \varphi} \frac{\partial f}{\partial \theta} .
$$

Thus integrating by parts, there holds

$$
\int_{\mathbb{T}} e_{1}\left(h_{n 1}\right) H(0) \mathrm{d} \sigma_{0}=\int_{0}^{2 \pi} d \theta \int_{0}^{2 \pi} \frac{\partial h_{n 1}}{\partial \varphi}(\sqrt{2}+2 \cos \varphi) d \varphi=\int_{0}^{2 \pi} d \theta \int_{0}^{2 \pi} h_{n 1} 2 \sin \varphi d \varphi .
$$


On the other hand, we see that

$$
\int_{\mathbb{T}} e_{2}\left(h_{n 2}\right) H(0) d \sigma_{0}=\int_{0}^{2 \pi} d \varphi \int_{0}^{2 \pi} \frac{1}{\sqrt{2}+\cos \varphi} \frac{\partial h_{n 2}}{\partial \theta}(\sqrt{2}+2 \cos \varphi) d \theta=0 .
$$

Therefore, one has

$$
\int_{\mathbb{T}}-4 \sum_{i=1}^{2} e_{i}\left(h_{n i}\right) H d \sigma_{0}=-8 \int_{0}^{2 \pi} \int_{0}^{2 \pi} h_{n 1} \sin \varphi d \varphi d \theta .
$$

Also, it follows from Lemma 4.3 that

$$
\sum_{i, j=1}^{2} h_{n j}\left\langle\nabla_{e_{i}} e_{i}, e_{j}\right\rangle H=h_{n 1}\left\langle\nabla_{e_{2}} e_{2}, e_{1}\right\rangle H=\frac{\sin \varphi}{\sqrt{2}+\cos \varphi} h_{n 1} H .
$$

Then we have

$$
\int_{\mathbb{T}} 4 \sum_{i, j=1}^{2} h_{n j}\left\langle\nabla_{e_{i}} e_{i}, e_{j}\right\rangle H d \sigma_{0}=\int_{0}^{2 \pi} \int_{0}^{2 \pi} 4 h_{n 1} \sin \varphi \frac{\sqrt{2}+2 \cos \varphi}{\sqrt{2}+\cos \varphi} d \varphi d \theta
$$

and

$$
\begin{aligned}
\int_{\mathbb{T}}-4 \sum_{i=1}^{2} e_{i}\left(h_{n i}\right) H+4 \sum_{i, j=1}^{2} h_{n j}\left\langle\nabla_{e_{i}} e_{i}, e_{j}\right\rangle H d \sigma_{0} & =4 \int_{0}^{2 \pi} \int_{0}^{2 \pi} h_{n 1}\left(-2+\frac{\sqrt{2}+2 \cos \varphi}{\sqrt{2}+\cos \varphi}\right) \sin \varphi d \theta d \varphi \\
& =-4 \int_{0}^{2 \pi} \int_{0}^{2 \pi} h_{n 1} \frac{\sqrt{2}}{\sqrt{2}+\cos \varphi} \sin \varphi d \theta d \varphi .
\end{aligned}
$$

Next, noting that

$$
\begin{aligned}
h_{n 1}= & -\frac{\operatorname{Sc}_{P}}{6}\langle X, n\rangle\left\langle X, e_{1}\right\rangle-\frac{1}{3}|X|^{2} \operatorname{Ric}_{P}\left(n, e_{1}\right)+\frac{1}{3}\left\{\langle X, n\rangle \operatorname{Ric}_{P}\left(X, e_{1}\right)+\left\langle X, e_{1}\right\rangle \operatorname{Ric}_{P}(X, n)\right\} \\
= & \frac{\operatorname{Sc}_{P}}{6} \sqrt{2} \sin \varphi(\sqrt{2} \cos \varphi+1)-\frac{1}{3}(3+2 \sqrt{2} \cos \varphi) \operatorname{Ric}_{P}\left(n, e_{1}\right) \\
& +\frac{1}{3}\left\{(\sqrt{2} \cos \varphi+1) \operatorname{Ric}_{P}\left(X, e_{1}\right)-\sqrt{2} \sin \varphi \operatorname{Ric}_{P}(X, n)\right\},
\end{aligned}
$$

one gets

$$
\begin{aligned}
\int_{0}^{2 \pi} h_{n 1} d \theta= & \frac{\sqrt{2}}{3} \pi \operatorname{Sc}_{P} \sin \varphi(\sqrt{2} \cos \varphi+1)-\frac{1}{3}(3+2 \sqrt{2} \cos \varphi) \pi\left(-\operatorname{Sc}_{P}+3 R_{33}\right) \cos \varphi \sin \varphi \\
& +\frac{1}{3}(\sqrt{2} \cos \varphi+1) \pi\left[-\operatorname{Sc}_{P}(\sqrt{2}+\cos \varphi) \sin \varphi+R_{33}\{3 \sin \varphi \cos \varphi+\sqrt{2} \sin \varphi\}\right] \\
& -\frac{\sqrt{2}}{3} \pi \sin \varphi\left[\operatorname{Sc}_{P}(\sqrt{2}+\cos \varphi) \cos \varphi+R_{33}\left\{2 \sin ^{2} \varphi-(\sqrt{2}+\cos \varphi) \cos \varphi\right\}\right] \\
= & -\frac{R_{33}}{3} \pi \sin \varphi(2 \cos \varphi+\sqrt{2}) .
\end{aligned}
$$

Substituting this equation into (65), we obtain

$$
\begin{aligned}
\int_{\mathbb{T}}-4 \sum_{i=1}^{2} e_{i}\left(h_{n i}\right) H+4 \sum_{i, j=1}^{2} h_{n j}\left\langle\nabla_{e_{i}} e_{i}, e_{j}\right\rangle H d \sigma_{0} & =\frac{4 \pi}{3} R_{33} \int_{0}^{2 \pi} \frac{(\sqrt{2}+2 \cos \varphi) \sqrt{2}}{\sqrt{2}+\cos \varphi} \sin ^{2} \varphi d \varphi \\
& =\frac{4 \pi}{3} R_{33} \int_{0}^{2 \pi} \sqrt{2}\left(2-\frac{\sqrt{2}}{\sqrt{2}+\cos \varphi}\right) \sin ^{2} \varphi d \varphi \\
& =\frac{8 \sqrt{2} \pi^{2}}{3} R_{33}-\frac{8 \pi}{3} R_{33} \int_{0}^{2 \pi} \frac{1-\cos ^{2} \varphi}{\sqrt{2}+\cos \varphi} d \varphi .
\end{aligned}
$$


Finally by Lemma 4.3 we obtain

$$
\begin{aligned}
\int_{\mathbb{T}}-4 \sum_{i=1}^{2} e_{i}\left(h_{n i}\right) H+4 \sum_{i, j=1}^{2} h_{n j}\left\langle\nabla_{e_{i}} e_{i}, e_{j}\right\rangle H d \sigma_{0} & =\frac{8 \sqrt{2}}{3} \pi^{2} R_{33}-\frac{8 \pi}{3} R_{33}(2 \sqrt{2} \pi-2 \pi) \\
& =\frac{8}{3} \pi^{2} R_{33}(2-\sqrt{2}) .
\end{aligned}
$$

Step 3: $-2 h_{n n} H^{2}+H^{2} \operatorname{tr}\left(h_{\mid T_{X} \mathbb{T}}\right)$.

By (62), one observes

$$
-2 h_{n n}=-\frac{\operatorname{Sc}_{P}}{3}\left\{|X|^{2}-\langle X, n\rangle^{2}\right\}+\frac{2}{3} \operatorname{Ric}_{P}(X, X)+\frac{2}{3}|X|^{2} \operatorname{Ric}(n, n)-\frac{4}{3}\langle X, n\rangle \operatorname{Ric}_{P}(X, n) .
$$

Since $\left\{e_{1}, e_{2}\right\}$ forms an orthonormal basis of $T_{X} \mathbb{T}$ and

$$
\left\langle h(X) e_{i}, e_{i}\right\rangle=\frac{\operatorname{Sc}_{P}}{6}\left\{|X|^{2}-\left\langle X, e_{i}\right\rangle^{2}\right\}-\frac{1}{3} \operatorname{Ric}_{P}(X, X)-\frac{1}{3}|X|^{2} \operatorname{Ric}_{P}\left(e_{i}, e_{i}\right)+\frac{2}{3}\left\langle X, e_{i}\right\rangle \operatorname{Ric}_{P}\left(X, e_{i}\right) \quad i=1,2,
$$

it follows from $\left\langle X, e_{2}\right\rangle=0$ that

$$
\begin{aligned}
& \operatorname{tr}\left(h_{\mid T_{X} \mathbb{T}}\right) \\
= & \frac{\operatorname{Sc}_{P}}{6}\left(2|X|^{2}-\left\langle X, e_{1}\right\rangle^{2}\right)-\frac{2}{3} \operatorname{Ric}_{P}(X, X)-\frac{|X|^{2}}{3}\left\{\operatorname{Ric}_{P}\left(e_{1}, e_{1}\right)+\operatorname{Ric}_{P}\left(e_{2}, \varepsilon_{2}\right)\right\}+\frac{2}{3}\left\langle X, e_{1}\right\rangle \operatorname{Ric}_{P}\left(X, e_{1}\right) .
\end{aligned}
$$

Since $\operatorname{Sc}_{P}=\operatorname{Ric}_{P}\left(e_{1}, e_{1}\right)+\operatorname{Ric}_{P}\left(e_{2}, e_{2}\right)+\operatorname{Ric}_{P}(n, n)$, we have

$$
\operatorname{tr}\left(h(X)_{\mid T_{X} \mathbb{T}}\right)=-\frac{\operatorname{Sc}_{P}}{6}\left\langle X, e_{1}\right\rangle^{2}-\frac{2}{3} \operatorname{Ric}_{P}(X, X)+\frac{1}{3}|X|^{2} \operatorname{Ric}_{P}(n, n)+\frac{2}{3}\left\langle X, e_{1}\right\rangle \operatorname{Ric}_{P}\left(X, e_{1}\right) .
$$

Combining (67) and (68) with $\left\langle X, e_{2}\right\rangle=0$ and $|X|^{2}-\langle X, n\rangle^{2}=\left\langle X, e_{1}\right\rangle^{2}$, we get

$$
\begin{aligned}
& -2 h_{n n}+\operatorname{tr}\left(h(X)_{\mid T_{X} \mathbb{T}}\right) \\
& =-\frac{\operatorname{Sc}_{P}}{2}\left\langle X, e_{1}\right\rangle^{2}+|X|^{2} \operatorname{Ric}_{P}(n, n)+\frac{2}{3}\left\langle X, e_{1}\right\rangle \operatorname{Ric}_{P}\left(X, e_{1}\right)-\frac{4}{3}\langle X, n\rangle \operatorname{Ric}_{P}(X, n) \\
& =-\operatorname{Sc}_{P} \sin ^{2} \varphi+(3+2 \sqrt{2} \cos \varphi) \operatorname{Ric}_{P}(n, n)-\frac{2 \sqrt{2}}{3} \sin \varphi \operatorname{Ric}_{P}\left(X, e_{1}\right)-\frac{4}{3}(\sqrt{2} \cos \varphi+1) \operatorname{Ric}_{P}(X, n) .
\end{aligned}
$$

Hence, from Lemma 4.3, there holds

$$
\begin{aligned}
& \int_{0}^{2 \pi}-2 h_{n n}+\operatorname{tr}\left(\left.h(X)\right|_{T_{X} \mathbb{T}}\right) d \theta \\
= & -2 \pi \operatorname{Sc}_{P} \sin ^{2} \varphi+(3+2 \sqrt{2} \cos \varphi) \pi\left[\operatorname{Sc}_{P} \cos ^{2} \varphi+R_{33}\left(2-3 \cos ^{2} \varphi\right)\right] \\
& -\frac{2 \sqrt{2}}{3} \pi \sin \varphi\left[-\operatorname{Sc}_{P}(\sqrt{2}+\cos \varphi) \sin \varphi+R_{33}\{\sqrt{2} \sin \varphi+3 \cos \varphi \sin \varphi\}\right] \\
& -\frac{4}{3} \pi(\sqrt{2} \cos \varphi+1)\left[\operatorname{Sc}_{P}(\sqrt{2}+\cos \varphi) \cos \varphi+R_{33}\left\{2 \sin ^{2} \varphi-(\sqrt{2}+\cos \varphi) \cos \varphi\right\}\right] \\
= & -\frac{\pi}{3} \operatorname{Sc}_{P}(\cos \varphi+\sqrt{2})^{2}+\frac{\pi}{3} R_{33}\{(\cos \varphi+\sqrt{2})(-3 \cos \varphi+5 \sqrt{2})-4\} .
\end{aligned}
$$

From this equality it follows that

$$
\begin{aligned}
& \int_{\mathbb{T}}\left\{-2 h_{n n}+\operatorname{tr}\left(h_{\mid T_{X} \mathbb{T}}\right)\right\} H^{2} d \sigma_{0} \\
= & \int_{0}^{2 \pi}\left[-\frac{\pi}{3} \operatorname{Sc}_{P}(\cos \varphi+\sqrt{2})(\sqrt{2}+2 \cos \varphi)^{2}\right] d \varphi \\
& +\int_{0}^{2 \pi} \frac{\pi}{3} R_{33}\left[\left\{(-3 \cos \varphi+5 \sqrt{2})-\frac{4}{\cos \varphi+\sqrt{2}}\right\}(\sqrt{2}+2 \cos \varphi)^{2}\right] d \varphi .
\end{aligned}
$$


Since

$$
\begin{aligned}
(\cos \varphi+\sqrt{2})(\sqrt{2}+2 \cos \varphi)^{2} & =4 \cos ^{3} \varphi+8 \sqrt{2} \cos ^{2} \varphi+10 \cos \varphi+2 \sqrt{2}, \\
(-3 \cos \varphi+5 \sqrt{2})(\sqrt{2}+2 \cos \varphi)^{2} & =-12 \cos ^{3} \varphi+8 \sqrt{2} \cos ^{2} \varphi+34 \cos \varphi+10 \sqrt{2}, \\
(\sqrt{2}+2 \cos \varphi)^{2} & =4 \cos \varphi(\cos \varphi+\sqrt{2})+2,
\end{aligned}
$$

we obtain

$$
\begin{aligned}
\int_{0}^{2 \pi}\left[-\frac{\pi}{3} \operatorname{Sc}_{P}(\cos \varphi+\sqrt{2})(\sqrt{2}+2 \cos \varphi)^{2}\right] d \varphi & =-\frac{\pi}{3} \operatorname{Sc}_{P}(8 \sqrt{2} \pi+4 \sqrt{2} \pi) \\
\int_{0}^{2 \pi} \frac{\pi}{3} R_{33}\left[\left\{(-3 \cos \varphi+5 \sqrt{2})-\frac{4}{\cos \varphi+\sqrt{2}}\right\}(\sqrt{2}+2 \cos \varphi)^{2}\right] d \varphi & =\frac{\pi}{3} R_{33}(8 \sqrt{2} \pi+20 \sqrt{2} \pi-16 \pi) .
\end{aligned}
$$

Thus we have

$$
\int_{\mathbb{T}}\left\{-2 h_{n n}+\operatorname{tr}\left(h_{\mid T_{X} \mathbb{T}}\right)\right\} H^{2} d \sigma_{0}=-4 \sqrt{2} \pi^{2} \operatorname{Sc}_{P}+\frac{\pi^{2}}{3}(28 \sqrt{2}-16) R_{33} .
$$

\section{Step 4: Conclusion}

By (64), (66) and (69), we obtain

$$
\dot{\tilde{W}}(0)=-4 \sqrt{2} \pi^{2}\left(\operatorname{Sc}_{P}-R_{33}\right)=-4 \sqrt{2} \pi^{2}\left(R_{11}+R_{22}\right)
$$

which implies (60). Thus we complete the proof.

Remark 4.4. Concerning the quantity $\operatorname{Sc}_{P}-\operatorname{Ric}_{P}(n, n)$, one can express it by the sectional curvature of the plane of symmetry of the Clifford torus at $P \in M$. In fact, choose an orthonormal basis $\left\{e_{1}, e_{2}, e_{3}\right\}$ of $T_{P} M$ with $e_{3}=n$. Denote by $K_{i j}$ the sectional curvature at $P \in M$ for the section spanned by $e_{i}, e_{j}$. Then from the relations

$$
R_{11}=K_{12}+K_{13}, \quad R_{22}=K_{12}+K_{23}, \quad R_{33}=K_{13}+K_{23}, \quad \mathrm{Sc}_{P}=R_{11}+R_{22}+R_{33},
$$

it follows that

$$
\operatorname{Sc}_{P}-\operatorname{Ric}_{P}(n, n)=\frac{1}{2} \operatorname{Sc}_{P}+K_{12} .
$$

\subsection{Asymptotics of the Willmore functional on degenerating tori}

The goal of this subsection is to understand the asymptotic behaviour of the Willmore functional for the degenerating tori, i.e., $\Sigma_{\varepsilon, P, R, \omega}$ when $|\omega|$ is close to 1 . For this purpose, let $\Sigma_{r} \subset T_{P} M$ be a round sphere of radius $r>0$ in the Euclidean metric $\left(\delta_{\alpha \beta}\right)$ which passes through the origin: we first compute the Willmore energy of $\Sigma_{r}$ with respect to the metric $g$. Recall that in normal coordinates, the metric $g$ is expressed as $g_{\alpha \beta}:=\delta_{\alpha \beta}+h_{\alpha \beta}(x)$ where $h$ satisfies

$$
|x|^{-2}|h(x)|+|x|^{-1}|\nabla h(x)|+\left|\nabla^{2} h(x)\right| \leq h_{0} ; \quad|x| \leq \rho
$$

for some $\rho, h_{0}>0$, see also (26). In the above setting, we prove

Lemma 4.5. Let $\Sigma_{r} \subset B_{\rho}(0)\left(\subset T_{P} M\right)$ be the round sphere of radius $r>0$ with respect to the Euclidean metric $\left(\delta_{\alpha \beta}\right)$ passing through the origin 0 . Then

$$
W_{g}\left(\exp _{P}^{g}\left(\Sigma_{r}\right)\right)=16 \pi-\frac{8 \pi}{3} \operatorname{Sc}_{P} r^{2}+O\left(r^{3}\right)
$$

where $\mathrm{Sc}_{P}$ is the scalar curvature of $(M, g)$ at $P$. 
Proof. Call $\left|\Sigma_{r}\right|_{g}$ and $\AA_{r}$ the area and the traceless second fundamental form of $\Sigma_{r}$ in metric $g$. Then from (70), one has

$$
\left|\Sigma_{r}\right|_{g}=4 \pi r^{2}+O\left(r^{4}\right) \text { and }\left|\AA_{r}\right|^{2}=O\left(r^{2}\right) .
$$

In fact, the first claim clearly holds by (70). The second claim also follows from (70) and the estimate (12) in 33 since the traceless second fundamental form of $\Sigma_{r}$ in the Euclidean space is null. Thus we obtain

$$
\mathcal{U}\left(\Sigma_{r}\right):=\int_{\Sigma_{r}}\left|\AA_{r}\right|^{2} d \sigma_{g}=O\left(r^{4}\right)<r\left|\Sigma_{r}\right|_{g}
$$

for $r$ small enough. Combining the area estimate in (71), the Conformal Willmore estimate (72) and the assumptions (70) on $h$, we are under the assumption of Theorem 5.1 in [21, therefore

$$
\left|W_{g}\left(\exp _{P}^{g}\left(\Sigma_{r}\right)\right)-16 \pi+\frac{2\left|\Sigma_{r}\right|_{g}}{3} \operatorname{Sc}_{P}\right| \leq C r\left|\Sigma_{r}\right|_{g}
$$

for some constant $C$ depending just on $h_{0}$. Recalling (71), the lemma follows.

Since the degenerating tori converge smoothly locally (outside the origin which is by construction the point of concentration of the shrinking handles) to a round sphere passing through the origin with radius $\sqrt[4]{2 \pi^{2}}$ by Proposition 2.5 (or Lemma 2.4), it is natural to expect the Willmore energy possesses an expansion accordingly to Lemma 4.5 more precisely we have the following proposition.

Proposition 4.6. There exists $C_{0}>0$, which is independent of $\varepsilon$, such that

$$
\limsup _{r \uparrow 1} \sup _{P \in M, R \in S O(3),|\omega|=r}\left|\frac{1}{\varepsilon^{2}}\left(W_{g_{\varepsilon}}\left(\Sigma_{\varepsilon, P, R, \omega}\right)-8 \pi^{2}+\frac{8 \sqrt{2}}{3} \pi^{2} \varepsilon^{2} \operatorname{Sc}_{P}\right)\right| \leq C_{0} \varepsilon
$$

for all sufficiently small $\varepsilon>0$.

Proof. First of all, we recall the scaling invariance of the Willmore functional, which in our case implies: $W_{g}\left(\exp _{P}(\Sigma)\right)=W_{g_{\varepsilon}}\left(\exp _{P}^{g_{\varepsilon}}\left(\varepsilon^{-1} \Sigma\right)\right)$. Hence, Lemma 4.5 yields

$$
W_{g_{\varepsilon}}\left(\exp _{P}^{g_{\varepsilon}}\left(R S_{\sqrt[4]{2 \pi^{2}}}, \sqrt[4]{2 \pi^{2}} \bar{\omega}\right)\right)=16 \pi-\frac{8 \sqrt{2}}{3} \pi^{2} \operatorname{Sc}_{P} \varepsilon^{2}+O\left(\varepsilon^{3}\right)
$$

for sufficiently small $\varepsilon$ and uniformly with respect to $P \in M, R \in S O(3)$ and $\bar{\omega}:=\omega /|\omega| \in S^{1}$. Here $S_{r_{1}, r_{2} \bar{\omega}}$ denotes the sphere centred at $r_{2} \bar{\omega}$ with radius $r_{1}$ for $r_{1}, r_{2}>0$ in the Euclidean space. Therefore, to prove the Proposition, it is enough to show that there exists $\varepsilon_{0}>0$ such that

$$
\limsup _{r \uparrow 1} \sup _{\varepsilon \in\left(0, \varepsilon_{0}\right], P \in M, R \in S O(3),|\omega|=r} \frac{1}{\varepsilon^{2}}\left|W_{g_{\varepsilon}}\left(\Sigma_{\varepsilon, P, R, \omega}\right)-W_{g_{\varepsilon}}\left(\exp _{P}^{g_{\varepsilon}}\left(R S \sqrt[4]{2 \pi^{2}}, \sqrt[4]{2 \pi^{2}} \bar{\omega}\right)\right)-8 \pi^{2}+16 \pi\right|=0 .
$$

We are going to estimate separately the errors in the handle part where the second fundamental form blows up as $|\omega| \uparrow 1$ and in the complementary region, where we have smooth convergence to a round sphere. To this end, for $\rho>0$, set

$$
\begin{array}{ll}
\Sigma_{\varepsilon, P, R, \omega, \rho, 1}:=\exp _{P}^{g_{\varepsilon}}\left(R \mathbb{T}_{\omega} \backslash B_{\rho}(0)\right), & \Sigma_{\varepsilon, P, R, \omega, \rho, 2}:=\exp _{P}^{g_{\varepsilon}}\left(R \mathbb{T}_{\omega} \cap B_{\rho}(0)\right), \\
\Sigma_{\varepsilon, P, R, \bar{\omega}, \rho, 1}:=\exp _{P}^{g_{\varepsilon}}\left(R S_{\sqrt[4]{2 \pi^{2}}, \sqrt[4]{2 \pi^{2}} \backslash} \backslash B_{\rho}(0)\right), & \Sigma_{\varepsilon, P, R, \bar{\omega}, \rho, 2}:=\exp _{P}^{g_{\varepsilon}}\left(R S_{\sqrt[4]{2 \pi^{2}}, \sqrt[4]{2 \pi^{2}} \bar{\omega}} \cap B_{\rho}(0)\right) .
\end{array}
$$

Notice that $\varepsilon=0$ corresponds to the Euclidean case, so the surface does not depend on $P \in M$. 
We first treat the sphere part, namely $\Sigma_{\varepsilon, P, R, \omega, \rho, 1}$. As in the previous subsection, let $t=\varepsilon^{2}, g_{t}$ as in (58) and

$$
W(t, \omega, \rho):=\int_{\Sigma_{\varepsilon, P, R, \omega, \rho, 1}} H_{g_{t}}^{2} d \sigma_{g_{t}} .
$$

Thanks to Lemma 2.4 we remark that for fixed $\rho>0, \Sigma_{\varepsilon, P, R, \omega, \rho, 1}$ converges to $\Sigma_{\varepsilon, P, R, \bar{\omega}, \rho, 1}$ smoothly and uniformly with respect to $(\varepsilon, P, R)$ as $\omega \rightarrow \bar{\omega}$. Therefore, for a suitable sequence $\left\{\rho_{r}\right\}$ satisfying $\rho_{r} \rightarrow 0$ as $r \rightarrow 1$, we observe that for some $t_{0}>0$

$$
\begin{aligned}
& \sup _{t \in\left(0, t_{0}\right), P \in M, R \in S O(3),|\omega|=r}\left|\frac{1}{t}\left[\left\{W\left(t, \omega, \rho_{r}\right)-W\left(0, \omega, \rho_{r}\right)\right\}-\left\{W\left(t, \bar{\omega}, \rho_{r}\right)-W\left(0, \bar{\omega}, \rho_{r}\right)\right\}\right]\right| \\
= & \sup _{t \in\left(0, t_{0}\right), P \in M, R \in S O(3),|\omega|=r}\left|\frac{1}{t} \int_{0}^{t}\left\{\frac{\partial W}{\partial s}\left(s, \omega, \rho_{r}\right)-\frac{\partial W}{\partial s}\left(s, \bar{\omega}, \rho_{r}\right)\right\} d s\right| \rightarrow 0
\end{aligned}
$$

as $r \rightarrow 1$. This implies that

$$
\begin{aligned}
\sup _{\varepsilon \in\left(0, \varepsilon_{0}\right), P \in M, R \in S O(3),|\omega|=r} \frac{1}{\varepsilon^{2}} \mid & \left\{W_{g_{\varepsilon}}\left(\Sigma_{\varepsilon, P, R, \omega, \rho_{r}, 1}\right)-W_{g_{0}}\left(\Sigma_{0, P, R, \omega, \rho_{r}, 1}\right)\right\} \\
& -\left\{W_{g_{\varepsilon}}\left(\Sigma_{\varepsilon, P, R, \bar{\omega}, \rho_{r}, 1}\right)-W_{g_{0}}\left(\Sigma_{0, P, R, \bar{\omega}, \rho_{r}, 1}\right)\right\} \mid \rightarrow 0
\end{aligned}
$$

as $r \rightarrow 1$.

Now we estimate the difference between $W_{g_{\varepsilon}}$ and $W_{g_{0}}$ in the handle region. We first remark that the surface $\Sigma_{\varepsilon, P, R, \omega, \rho_{r}, 2}$ is diffeomorphic to $R \mathbb{T}_{\omega} \cap B_{\rho_{r}}(0)$, hence we regard all geometric quantities of $\Sigma_{\varepsilon, P, R, \omega, \rho_{r}, 2}$ as functions on $R \mathbb{T}_{\omega} \cap B_{\rho_{r}}(0)$. By applying [33, Lemma 2.3 with the choice $\gamma=\varepsilon^{2} \rho_{r}^{2}$ ] and recalling (28)-(29), we get

$$
\left|H_{g_{\varepsilon}}^{2}-H_{g_{0}}^{2}\right| \leq C_{0} \varepsilon^{2}\left(\rho_{r}^{2}\left|A_{g_{0}}\right|^{2}+1\right) \quad \text { on } R \mathbb{T}_{\omega} \cap B_{\rho_{r}}(0),
$$

where $H_{g_{0}}$ is mean curvature of $R \mathbb{T}_{\omega} \cap B_{\rho_{r}}(0)$ in metric $g_{\varepsilon}$ and $H_{g_{0}}, A_{g_{0}}$ are the mean curvature and the second fundamental form of $R \mathbb{T}_{\omega} \cap B_{\rho_{r}}(0)$ in the Euclidean metric, and $C_{0}>0$ is a constant which does not depend on $\varepsilon, P, R, \omega$. Recalling also the following estimate of the ratio of the area forms $d \sigma_{g_{\varepsilon}}$ and $d \sigma_{g_{0}}$ in metric $g_{\varepsilon}$ and Euclidean metric (see [33, Lemma 2.2])

$$
\left(1-4 C_{0} \rho_{r}^{2} \varepsilon^{2}\right) d \sigma_{g_{0}} \leq d \sigma_{g_{\varepsilon}} \leq\left(1+4 C_{0} \rho_{r}^{2} \varepsilon^{2}\right) d \sigma_{g_{0}} \quad \text { on } R \mathbb{T}_{\omega} \cap B_{\rho_{r}}(0),
$$

it follows that

$$
\begin{aligned}
\frac{1}{\varepsilon^{2}}\left|W_{g_{\varepsilon}}\left(\Sigma_{\varepsilon, P, R, \omega, \rho_{r}, 2}\right)-W_{g_{0}}\left(\Sigma_{0, P, R, \omega, \rho_{r}, 2}\right)\right| & \leq C_{1} \int_{R \mathbb{T}_{\omega} \cap B_{\rho_{r}}(0)}\left(\rho_{r}^{2}\left|A_{g_{0}}\right|^{2}+1\right) d \sigma_{g_{0}} \\
& \leq C_{1} \rho_{r}^{2} \int_{R \mathbb{T}_{\omega}}\left|A_{g_{0}}\right|^{2} d \sigma_{g_{0}}+C_{1}\left|R \mathbb{T}_{\omega} \cap B_{\rho_{r}}(0)\right|_{g_{0}} .
\end{aligned}
$$

Noting the conformal invariance of $\int_{\Sigma}\left|A_{g_{0}}\right|^{2} d \sigma_{g_{0}}$ in the Euclidean space, $\rho_{r} \rightarrow 0$ and $\left|\mathbb{T}_{r \mathbf{e}_{x}} \cap B_{\rho_{r}}(0)\right|_{g_{0}} \rightarrow 0$ due to Lemma 2.4, we obtain

$$
\begin{aligned}
& \sup _{\varepsilon \in\left(0, \varepsilon_{0}\right), P \in M, R \in S O(3),|\omega|=r} \frac{1}{\varepsilon^{2}}\left|W_{g_{\varepsilon}}\left(\Sigma_{\varepsilon, P, R, \omega, \rho_{r}, 2}\right)-W_{g_{0}}\left(\Sigma_{0, P, R, \omega, \rho_{r}, 2}\right)\right| \\
\leq & C_{1} \rho_{r}^{2} \int_{\mathbb{T}}\left|A_{g_{0}}\right|^{2} d \sigma_{g_{0}}+C_{1}\left|\mathbb{T}_{r \mathbf{e}_{x}} \cap B_{\rho_{r}}(0)\right|_{g_{0}} \rightarrow 0 \quad \text { as } r \rightarrow 1 .
\end{aligned}
$$

In an analogous way, but it is actually easier since the second fundamental form is bounded, one can check that

$$
\sup _{\varepsilon \in\left(0, \varepsilon_{0}\right), P \in M, R \in S O(3), \bar{\omega} \in S^{2}} \frac{1}{\varepsilon^{2}} \mid W_{g_{\varepsilon}}\left(\Sigma_{\varepsilon, P, R, \bar{\omega}, \rho_{r}, 2}\right)-W_{g_{0}}\left(\Sigma_{\left.0, P, R, \bar{\omega}, \rho_{r}, 2\right)}\right) \rightarrow 0 \quad \text { as } r \rightarrow 1 .
$$

Finally, since the conformal invariance of the Willmore functional in the Euclidean space gives

$$
16 \pi=W_{g_{0}}\left(\Sigma_{0, P, R, \bar{\omega}, \rho_{r}, 1}\right)+W_{g_{0}}\left(\Sigma_{0, P, R, \bar{\omega}, \rho_{r}, 2}\right), \quad 8 \pi^{2}=W_{g_{0}}\left(\Sigma_{0, P, R, \omega, \rho_{r}, 1}\right)+W_{g_{0}}\left(\Sigma_{0, P, R, \omega, \rho_{r}, 2}\right),
$$

combining (74), (75) and (76), the claim (73) follows and we complete the proof. 


\section{Proof of the main theorems}

In this short section, collecting the above results, we prove Theorems 1.1 and 1.6 starting from the former.

\subsection{Proof of Theorem 1.1}

First of all, from (31), we notice that

$$
\partial \mathcal{T}_{K, \varepsilon}=\left\{\exp _{P}^{g_{\varepsilon}}\left(R \mathbb{T}_{\omega}\right): P \in M, R \in S O(3), \omega \in \partial K\right\} .
$$

Combining Propositions 3.6 and 3.7 we know that, for every compact subset $K \subset \subset \mathbb{D}$ to be fixed later, there exists $\bar{\varepsilon}_{K}>0$ such that for every $\varepsilon \in\left(0, \bar{\varepsilon}_{K}\right]$ there is a function $\Phi_{\varepsilon}: \mathcal{T}_{K, \varepsilon} \rightarrow \mathbb{R}$ with the following properties:

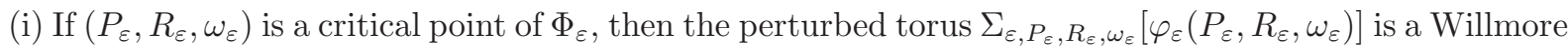

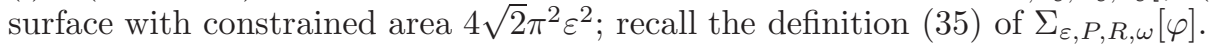

(ii) There exists a constant $C_{K}>0$ depending just on $K$ such that, for every $(P, R, \omega) \in M \times S O(3) \times K$, it holds

$$
\left|\Phi_{\varepsilon}(P, R, \omega)-W_{g_{\varepsilon}}\left(\Sigma_{\varepsilon, P, R, \omega}\right)\right| \leq C_{K} \varepsilon^{4}
$$

In order to choose the compact subset $K \subset \subset \mathbb{D}$ suitably, let us recall the expansion of the Willmore functional on symmetric tori and on degenerate tori (given in Propositions 4.1 and 4.6 respectively).

(iii) For every $P \in M$ and $\varepsilon$ small enough it holds

$$
W_{g_{\varepsilon}}\left(\Sigma_{\varepsilon, P, R, 0}\right)=8 \pi^{2}-4 \sqrt{2} \pi^{2}\left\{\operatorname{Sc}_{P}-\operatorname{Ric}_{P}(R n, R n)\right\} \varepsilon^{2}+O\left(\varepsilon^{3}\right),
$$

where $n \in T_{P} M$ denotes the normal axial vector of $\mathbb{T}$.

(iv) There exist $\varepsilon_{0}>0$ and $C_{0}>0$ such that

$$
\limsup _{r \uparrow 1} \sup _{P \in M, R \in S O(3),|\omega|=r} \frac{1}{\varepsilon^{2}}\left|W_{g}\left(\Sigma_{\varepsilon, P, R, \omega}\right)-8 \pi^{2}+\frac{8 \sqrt{2}}{3} \pi^{2} \varepsilon^{2} \mathrm{Sc}_{P}\right| \leq C_{0} \varepsilon
$$

for all $\varepsilon \in\left(0, \varepsilon_{0}\right]$. If we now assume (as in the hypotheses of Theorem 1.1]) that

$$
3 \sup _{P \in M}\left(\operatorname{Sc}_{P}-\inf _{|\nu|_{g}=1} \operatorname{Ric}_{P}(\nu, \nu)\right)>2 \sup _{P \in M} \operatorname{Sc}_{P},
$$

(or, respectively $\left.3 \inf _{P \in M}\left(\operatorname{Sc}_{P}-\sup _{|\nu| g=1} \operatorname{Ric}_{P}(\nu, \nu)\right)<2 \inf _{P \in M} \operatorname{Sc}_{P}\right)$ then, combining (ii), (iii), (iv) above there exists $r \in(0,1), \varepsilon_{1}>0$ such that, chosen as compact subset $K:=B_{r}(0) \subset \subset \mathbb{D}$, the corresponding reduced functional $\Phi_{\varepsilon}$ satisfies

$$
\inf _{\mathcal{T}_{K, \varepsilon}} \Phi_{\varepsilon}<\inf _{\partial \mathcal{T}_{K, \varepsilon}} \Phi_{\varepsilon} \quad\left(\text { or, respectively, } \sup _{\mathcal{T}_{K, \varepsilon}} \Phi_{\varepsilon}>\sup _{\partial \mathcal{T}_{K, \varepsilon}} \Phi_{\varepsilon}\right),
$$

for every $\varepsilon \in\left(0, \varepsilon_{1}\right]$.

It follows that the global minimum of $\Phi_{\varepsilon}$ (resp. the global maximum) is achieved at an interior point of $\mathcal{T}_{K, \varepsilon}$, which therefore is a critical point of $\Phi_{\varepsilon}$. By recalling (i) above we conclude that the perturbed torus $\Sigma_{\varepsilon, P_{\varepsilon}, R_{\varepsilon}, \omega_{\varepsilon}}\left[\varphi_{\varepsilon}\left(P_{\varepsilon}, R_{\varepsilon}, \omega_{\varepsilon}\right)\right]$ is a Willmore surface with constrained area $4 \sqrt{2} \pi^{2} \varepsilon^{2}$ and finally, by construction, the graph function $\varphi_{\varepsilon}$ converges to 0 in $C^{4, \alpha}$-norm as $\varepsilon \rightarrow 0$ with decay rate $\varepsilon^{2}$ in the rescaled metric $g_{\varepsilon}$. This concludes the proof.

Remark 5.1 (Proof of Remark 1.2). Notice that if both the conditions (3) and (4) are satisfied then, by repeating verbatim the proof above, the reduced functional $\Phi_{\varepsilon}$ has both global minimum and global maximum attained at interior points of $\mathcal{T}_{K, \varepsilon}$. It follows that exist at least two smooth embedded Willmore tori in $(M, g)$ with constrained area equal to $4 \sqrt{2} \pi^{2} \varepsilon^{2}$. 


\subsection{Proof of Theorem 1.6}

The only additional difficulty in proving Theorem 1.6 is that we have to find both a compact subset of the unit disk $\mathbb{D}$ and a compact subset of $\mathbb{R}^{3} \backslash\{0\}$ where to perform the finite dimensional reduction. Let us discuss the latter, the former being analogous to the compact case.

As recalled in the introduction, the Schwarzschild metric $g_{i j}$ on $\mathbb{R}^{3} \backslash\{0\}$ is conformal to the Euclidean one, its scalar curvature vanishes identically and it has two asymptotically flat ends (one for $r \downarrow 0^{+}$and one for $r \uparrow+\infty)$. For $\tau>0$, set

$$
A_{\tau}:=\left\{P \in \mathbb{R}^{3}: \tau \leq|P|_{g_{0}} \leq \tau^{-1}\right\}, \quad \eta:=\max _{\mathbb{R}^{3} \backslash\{0\}} \max _{|\nu|_{g_{S c h}}=1}\left|\operatorname{Ric}_{P}(\nu, \nu)\right|>0 .
$$

Remark that the positivity of $\eta$ easily follows from either direct computations or observing that Ric $\equiv 0$ implies that $\left(\mathbb{R}^{3} \backslash\{0\}, g_{S c h}\right)$ is isometric to the Euclidean space. In addition, since $\left(\mathbb{R}^{3} \backslash\{0\}, g_{S c h}\right)$ has two flat ends, there exists a $\tau_{0}>0$ such that $\eta$ is achieved at interior points $P$ of $A_{\tau}$ provided $\tau \in\left(0, \tau_{0}\right)$. Furthermore, noting that $W_{g_{0}}\left(R \mathbb{T}_{\omega}\right)=W_{g_{0}}(\mathbb{T})=8 \pi^{2}$ by conformal invariance of $W_{g_{0}}$, for every compact set $K \subset \mathbb{D}$, one can find $C_{K}>0$ such that if either $|P|_{g_{0}} \leq \tau$ or $\tau^{-1} \leq|P|_{g_{0}}$, then

$$
\begin{aligned}
\sup _{P \in A_{\tau}^{c}, R \in S O(3), \omega \in K}\left|W_{g_{\varepsilon}}\left(\exp _{P}^{g_{\varepsilon}}\left(R \mathbb{T}_{\omega}\right)\right)-8 \pi^{2}\right| & =\sup _{P \in A_{\tau}^{c}, R \in S O(3), \omega \in K}\left|W_{g_{\varepsilon}}\left(\exp _{P}^{g_{\varepsilon}}\left(R \mathbb{T}_{\omega}\right)\right)-W_{g_{0}}\left(R \mathbb{T}_{\omega}\right)\right| \\
& \leq C_{K} O_{\tau}(1) \varepsilon^{2}
\end{aligned}
$$

where $o_{\tau}(1) \rightarrow 0$ as $\tau \rightarrow 0$. As in Proposition 4.6, by Sc $\equiv 0$ on $\mathbb{R}^{3} \backslash\{0\}$, we may also prove that

$$
\limsup _{r \rightarrow 1} \sup _{P \in \mathbb{R}^{3} \backslash\{0\}, R \in S O(3),|\omega|=r}\left|\frac{1}{\varepsilon^{2}}\left(W_{g_{\varepsilon}}\left(\Sigma_{\varepsilon, P, R, \omega}[0]\right)-8 \pi^{2}\right)\right| \leq C_{0} \varepsilon .
$$

Hence, we may find $r_{\eta} \in(0,1)$ and $\varepsilon_{\eta}>0$ such that if $\tau \in\left(0, \tau_{0}\right)$ and $\varepsilon \in\left(0, \varepsilon_{\eta}\right)$, then

$$
\sup _{P \in A_{\tau}, R \in S O(3),|\omega|=r_{\eta}}\left|W_{g_{\varepsilon}}\left(\exp _{P}^{g_{\varepsilon}}\left(R \mathbb{T}_{\omega}\right)\right)-8 \pi^{2}\right| \leq \frac{\eta}{4} \varepsilon^{2}
$$

Setting $K:=\overline{B_{r_{\eta}}(0)}$, by (77), there exists a $\tau_{1, \eta} \in\left(0, \tau_{0}\right)$ satisfying

$$
\sup _{P \in \partial A_{\tau_{1, \eta}}, R \in S O(3), \omega \in K}\left|W_{g_{\varepsilon}}\left(\exp _{P}^{g_{\varepsilon}}\left(R \mathbb{T}_{\omega}\right)\right)-8 \pi^{2}\right| \leq \frac{\eta}{2} \varepsilon^{2}
$$

for all $\varepsilon \in\left(0, \varepsilon_{\eta}\right)$.

Now one can repeat the proof of Theorem 1.1 by replacing the ambient space $M$ with $A_{\tau_{1, \eta}}$ above, getting a compact subset $K:=\overline{B_{r_{\eta}}(0)} \subset \subset \mathbb{D}$ and an $\varepsilon_{0}>0$ such that the corresponding reduced functional $\Phi_{\varepsilon}$ has an interior critical point. Therefore there exists a perturbed torus $\Sigma_{\varepsilon, P_{\varepsilon}, R_{\varepsilon}, \omega_{\varepsilon}}\left[\varphi_{\varepsilon}\left(P_{\varepsilon}, R_{\varepsilon}, \omega_{\varepsilon}\right)\right]$ which is a Willmore surface with constrained area $4 \sqrt{2} \pi^{2} \varepsilon^{2}$ whose graph function $\varphi_{\varepsilon}$ converges to 0 in $C^{4, \alpha_{-}}$ norm as $\varepsilon \rightarrow 0$. We conclude by recalling that the area constrained-Willmore surfaces coincide with the critical points of the Hawking mass under area constraint and that the rotational invariance of the Scwarzschild metric implies that all the surfaces obtained by rotating $\Sigma_{\varepsilon, P_{\varepsilon}, R_{\varepsilon}, \omega_{\varepsilon}}\left[\varphi_{\varepsilon}\left(P_{\varepsilon}, R_{\varepsilon}, \omega_{\varepsilon}\right)\right]$ around the origin $0 \in \mathbb{R}^{3}$ are critical points as well.

Remark 5.2. The generalization to ALE scalar flat 3-manifods claimed in Remark 1.7 follows on the same line of the proof above once we replace the annulus $A_{\tau}$ by a large ball $B_{\tau}\left(x_{0}\right)$, for some fixed $x_{0} \in M$. Indeed, assumptions 1)-2) imply that

$$
\sup _{P \in B_{\tau}\left(x_{0}\right)^{c}, R \in S O(3), \omega \in K}\left|W_{g_{\varepsilon}}\left(\exp _{P}^{g_{\varepsilon}}\left(R \mathbb{T}_{\omega}\right)\right)-8 \pi^{2}\right| \leq C_{K} o_{\tau}(1) \varepsilon^{2}
$$

where $o_{\tau}(1) \rightarrow 0$ as $\tau \rightarrow 0$; the rest of the proof can be repeated verbatim. 


\section{References}

[1] A. Ambrosetti, M. Badiale, Homoclinics: Poincaré-Melnikov type results via a variational approach, Ann. Inst. Henri Poincaré Analyse Non Linèaire 15, (1998), 233-252 .

[2] A. Ambrosetti, M. Badiale, Variational perturbative methods and bifurcation of bound states from the essential spectrum Proc. R. Soc. Edinb. 18, (1998), 1131-1161.

[3] A. Ambrosetti, A. Malchiodi, Perturbation methods and semilinear elliptic problems in $\mathbb{R}^{n}$, Progress in mathematics, Birkhauser, (2006).

[4] M. Barros, A. Ferrández, P. Lucas, M. A. Merono, Willmore tori and Willmore-Chen submanifolds in pseudo-Riemannian spaces, J. Geom. Phys., Vol. 28, (1998), 45-66.

[5] M. Bauer, E. Kuwert, Existence of minimizing Willmore surfaces of prescribed genus, Int. Math. Res. Not., Vol.10, (2003), 553-576.

[6] A. L. Besse, Einstein Manifolds, Classics in Mathematics, Springer-Verlag, Berlin, 2008 (Reprint of the 1987 edition), xii +516 .

[7] H. Bray, The Penrose inequality in general relativity and volume comparison theorems involving scalar curvature, Ph.D. thesis, Stanford University (1997), arXiv:0902.3241,

[8] S. Brendle, Constant mean curvature surfaces in warped product manifolds, Publ. Math. de l'IHÉS $117,247-269,(2013)$.

[9] S. Brendle, M. Eichmair, Isoperimetric and Weingarten surfaces in the Schwarzschild manifold, Journ. Diff. Geom., Vol. 94, 387-407, (2013)

[10] A. Carlotto, A. Mondino, Existence of Generalized Totally Umbilic 2-Spheres in Perturbed 3-Spheres Int. Math. Res Not., 21, 6020-6052, (2014).

[11] J. Chen, Y. Li, Bubble tree of branched conformal immersions and applications to the Willmore functional, Amer. J. Math., Vol. 136, Num. 4, (2014), 1107-1154.

[12] D. Christodoulou, S.-T. Yau, Some remarks on the quasi-local mass, In: Mathematics and general relativity, Contemporary Math., Vol. 71, 9-14, (1986).

[13] N. Ikoma, A. Malchiodi, A. Mondino, Embedded area-constrained Willmore tori of small area in Riemannian three-manifolds II: Morse Theory, preprint arXiv:1411.4412 to appear in Amer. J. Math.

[14] R. Kusner, Estimates for the biharmonic energy on unbounded planar domains, and the existence of surfaces of every genus that minimize the squared-mean-curvature integral, Elliptic and Parabolic Methods in Geometry (Minneapolis, Minn, 1994), A. K. Peters, Massachusetts, (1996), 67-72.

[15] E. Kuwert, A. Mondino, J. Schygulla, Existence of immersed spheres minimizing curvature functionals in compact 3-manifolds, Math. Ann. 359 (2014), no. 1-2, 379-425.

[16] E. Kuwert, R. Schätzle, Removability of isolated singularities of Willmore surfaces, Annals of Math. Vol. 160, Num. 1, (2004), 315-357.

[17] T. Lamm, J. Metzger, Small surfaces of Willmore type in Riemannian manifolds, Int. Math. Res. Not. 19 (2010), 3786-3813.

[18] T. Lamm, J. Metzger, Minimizers of the Willmore functional with a small area constraint, Annales IHP-Anal. Non Lin., Vol. 30, (2013), 497-518.

[19] T. Lamm, J. Metzger, F. Schulze, Foliations of asymptotically flat manifolds by surfaces of Willmore type, Math. Ann., Vol. 350, Num. 1, (2011), 1-78. 
[20] S. Lang, Undergraduate analysis. Second edition. Undergraduate Texts in Mathematics. SpringerVerlag, New York, 1997.

[21] P. Laurain, A. Mondino, Concentration of small Willmore spheres in Riemannian 3-manifolds, Analysis \& PDE, Vol. 7, Num. 8, (2014), 1901-1921.

[22] J. M. Lee, T. H. Parker, The Yamabe problem, Bull. Amer. Math. Soc. (N.S.), Vol. 17, Num. 1, (1987), 37-91.

[23] P. Li, S. T. Yau, A new conformal invariant and its applications to the Willmore conjecture and the first eigenvalue of compact surfaces, Invent. Math., Vol. 69, Num. 2, (1982), 269-291.

[24] J. M. Manzano, R. Souam The classification of totally umbilical surfaces in homogeneous 3-manifolds, Math. Zeit., Vol. 279, (2015), Num. 1-2, 557-576.

[25] F. Marques, A. Neves, Min-max theory and the Willmore conjecture, Ann. Math., (2) 179 (2014), Num. 2, 683-782.

[26] W. H. Meeks III, J. Perez, Costant mean curvature surfaces in metric Lie groups, "Geometric Analysis: Partial Differential Equations and Surfaces", Contemporary Mathematics (AMS), Vol. 570, (2012), 25-110.

[27] W. H. Meeks III, P. Mira, J. Perez, A. Ros, Constant mean curvature spheres in homogeneous three-spheres, Preprint arXiv:1308.2612, (2013).

[28] A. Mondino, Some results about the existence of critical points for the Willmore functional, Math. Zeit., Vol. 266, Num. 3, (2010), 583-622.

[29] A. Mondino, The conformal Willmore Functional: a perturbative approach, J. Geom. Anal., Vol. 23, Num. 2, (2013), 764-811.

[30] A. Mondino, H. T. Nguyen, A Gap Theorem for Willmore Tori and an application to the Willmore Flow, Nonlinear Analysis TMA, Vol. 102, (2014), 220-225.

[31] A. Mondino, T. Rivière, Immersed Spheres of Finite Total Curvature into Manifolds, Advances Calc. Var., Vol. 7, Num. 4, (2014), 493-538.

[32] A. Mondino, T.Rivière, Willmore spheres in compact Riemannian manifolds, Advances Math., Vol. 232, Num. 1, (2013), 608-676.

[33] A. Mondino, J. Schygulla, Existence of immersed spheres minimizing curvature functionals in noncompact 3-manifolds, Annales IHP-Anal. Non Lin., Vol. 31, (2014), 707-724.

[34] S. Montiel, A. Ros, Minimal immersions of surfaces by the first Eigenfunctions and conformal area, Invent. Math., Vol. 83, (1986), 153-166.

[35] F. Pacard, X. Xu, Constant mean curvature spheres in Riemannian manifolds, Manuscripta Math. Vol.128, 275?295 (2008).

[36] T. Rivière, Analysis aspects of Willmore surfaces, Invent. Math., Vol. 174, Num. 1, (2008), 1-45.

[37] T. Rivière, Variational Principles for immersed Surfaces with $L^{2}$-bounded Second Fundamental Form, J. Reine Angew. Math., Vol. 695, (2014), 41-98.

[38] A. Ros, The Willmore conjecture in the real projective space, Math. Res. Lett., Vol. 6, (1999), 487493.

[39] L. Simon, Existence of surfaces minimizing the Willmore functional, Comm. Anal. Geom., Vol. 1, Num. 2, (1993), 281-325. 
[40] P. Topping, Towards the Willmore conjecture. Calc. Var. and PDE, Vol. 11, (2000), 361-393.

[41] F. Urbano, Minimal surfaces with low index in the three-dimensional sphere, Proc. Amer. Math. Soc., Vol. 108, (1990), 989-992.

[42] P. Wang, On the Willmore functional of 2-tori in some product Riemannian manifolds, Glasgow Math. J., Vol. 54, (2012), 517-528.

[43] J. L. Weiner, On a problem of Chen, Willmore, et al., Indiana Univ. Math. J., Vol 27, Num. 1, (1978), 19-35.

[44] T.J. Willmore, Riemannian Geometry, Oxford Science Publications, Oxford University Press (1993). 